\title{
DYNAMO ACTION IN THE IONOSPHERE AND MOTIONS OF THE MAGNETOSPHERIC PLASHA
}

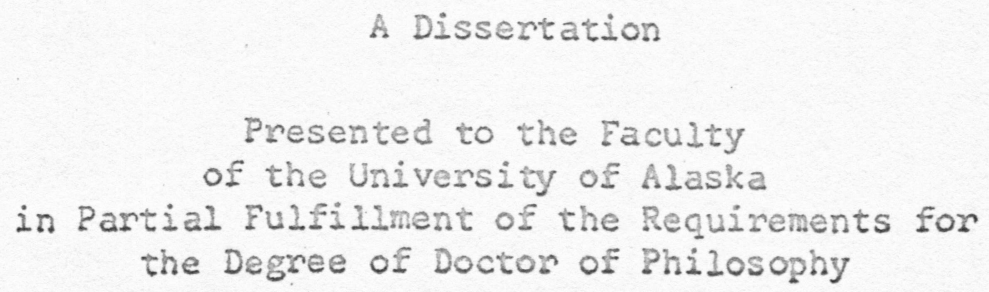

Ronald N. Delitt, B.S., M.S.

May 1965

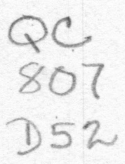


DYNAMO ACTION IN THE IONOSPHERE AND MOTIONS

OF THE MAGNETOSPHERIC PLASMA

APPROVED:

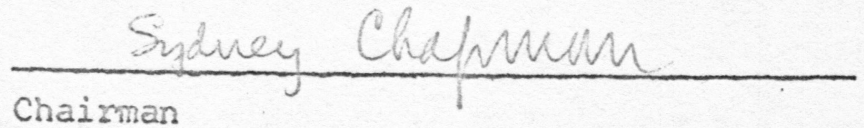

Chaiman
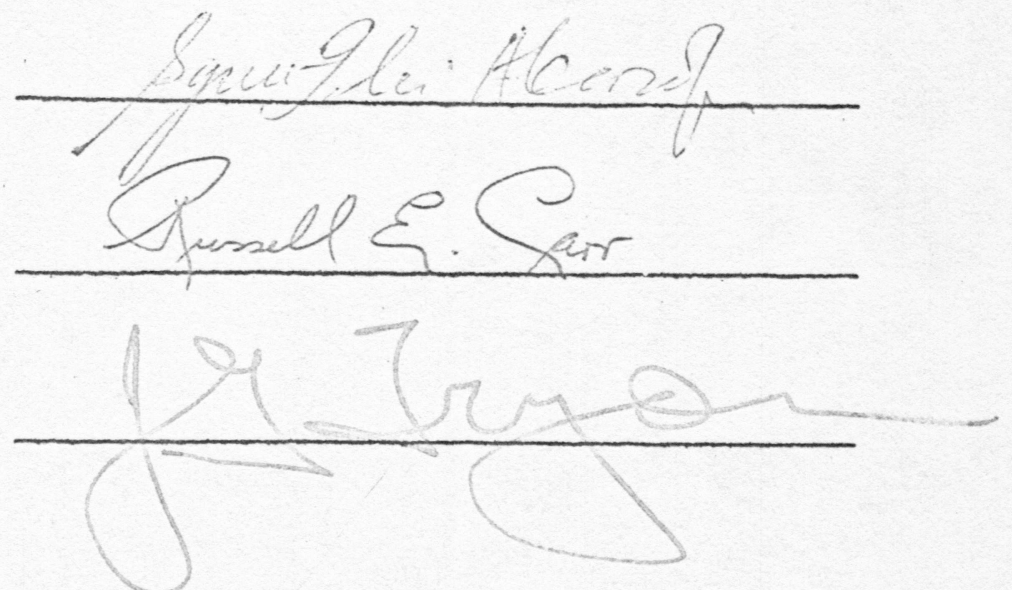

APPROVED:

Dean of the College of Mathematics, Physical Science, and Engineering

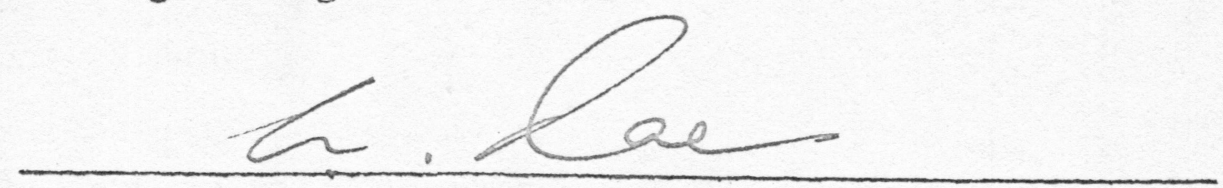

Vice President for Research and Advanced Study 
This thesis presents a study of the dynamic interaction which takes place between the magnetospheric plasma and the underlying neutral atmosphere; it is hoped thus to gain a better understanding of the effects of this interaction upon the steady state configuration of the magnetosphere.

The neutral portion of the atmosphere (the neutrosphere) and the overlying ionized regions (the upper atmosphere and magnetosphere) may be regarded as two distinct dynamic domains that interact in a region of transition occurring between 100 and $150 \mathrm{~km}$ over the earth. The neutrosphere because of its greater mass will dominate the motion, and the magnetospheric plasma can be expected to undergo motions related to those of the upper neutrosphere and transition region. However, the geomagnetic field restricts the motion of the magnetospheric plasma to a particular class, allowing one to consider the magnetospheric motion to be constrained.

Motions in the transition region of the class not permitted the magnetospheric plasma will give rise to forces against the constraint. The reaction of the constraint on the atmosphere of the transition region takes the form of a Lorentz force $J \times B$ where $J$ is the current responsible for the well known solar quiet day daily magnetic variation $(\mathrm{Sq})$. The explanation for the production of this current in the transition region has traditionally been presented in terms of a dynamo-like electromotive force generated by motions of the conducting atmosphere through the magnetic field, whence the transition region is aptly named the dynamo region. 
The Lorentz force represented by this current constitutes a significant term in the equation of motion for the dynamo region. Another important term arises from eddy viscous stresses immediately below the dynamo region. The equation of motion for the dynamo region must thus include such forces as well as the pressure gradient and Coriolis terms. However, our almost total ignorance of the eddy viscous stress field at the lower surface of the dynamo layer at present precludes our deducing the entire dynamo layen winds from the observed Sq magnetic variation.

The kinematics of the dynamo layer are discussed and the motion of the dynamo layer is divided into a symmetric and an antisymmetric part. The term symmetric is here used to describe winds in the northern and southern hemisphere that are the mirror images of each other with respect to the equatorial plane. It is demonstrated that the symmetric component gives rise to electrostatic fields transverse to the field lines, but to no currents along the field lines, while the antisymmetric case produces the converse effects. The symmetric and antisymmetric winds are further divided into components according to the horizontal electromotive force they produce.

\section{(a) Symmetric Wind}

In the case of the symmetric wind, only the portion of the wind producing the solenoidal component of the horizontal dynamo electromotive force is effective in producing ionospheric currents. It is demonstrated that only this current producing wind system acts against the constraints imposed by the geomagnetic field on magnetospheric motions. The motion of the magnetospheric plasma driven by each such wind system is discussed. 
The earlier treatments of the dynamo theory consider the dynamo region to be a single layer in which the wind system and the electric conductivity are assumed to be uniform in height. A new, more general derivation of the layer's dynamo action is given in which no restrictions are placed upon the vertical distributions. An effective wind is defined which permits the use of the earlier equations relating the current function, the electrostatic field, and the scalar field describing the current producing part of the effective wind. The equation relating the electrostatic field and the current function is essentially that employed by Maeda (1956), allowing his solution for the portion of the electrostatic field associated with the current producing wind to remain unaffected by the stratification of the wind system.

Mathematical techniques for solving the dynamo equations for the elecrostatic field are developed. These allow for a quite general conductivity distribution over the globe, only requiring that it be expressible in surface hamonics. The effect of undetected zonal currents upon the solution for the electrostatic field is discussed. It is suggested that a considerable diumal component of electrostatic field and other components as well may be hidden from us by our inability to detect the prevailing magnetic perturbations produced by zonal currents. The electrostatic field associated with the non-current producing components of the symmerric wind is likewise hidden from us.

\section{(b) Antisymmetric Wind}

The equations for the current driven by the antisymmetric component of wind are derived, and some of the effects of such currents are discussed. It is found that the conduction of current along the field lines 
from one hemisphere to the other is associated with an interhemispheric stress between geomagnetically conjugate points of order $3 \times 10^{-7}$ newtons/meter ${ }^{2}$.

In addition it is found that an antisymmetric layer current density of 5 amperes $/ \mathrm{km}$ into the polar cap region (across the $75^{\circ}$ latitude circle) might give rise to a displacement of about $150 \mathrm{~km}$ in the relative position of the conjugate points defined by field lines of the magnetospheric tail.

It is suggested that the dynamo action in the 100 to $150 \mathrm{~km}$ height plays a role in determining the manner in which the magnetosphere divides itself into the conotating region and the magnetospheric tail. 


\section{ACKNONEDGENENIS}

The author wishes to thank the members of his committee, Dr. Sydney Chapmen, Dr. Syun-Ichi Akasofu, and Dr. Russell Carr for their encouragement. I am especially indebted to Dr. Akasofu for much advice and guidance in the course of the research and to Dr. Carr for carefuliy going over the mathematical developments in the manuscript.

I would also like to acknowledge the helpful discussions and encouragement of Dr. Bemhand Haurwitz.

I am grateful to Jean Chorbajian and Helen kendris, not only for bridging the gap between theager instructions and the realization of numerical results from the computer, but also for helpful comments with regard to the mathematical development presented in the thesis.

Credit for drafting the figures goes to Peter Koelsch and to Ann Dupere for supervising the typing and printing of the manuscript. Great appreciation is felt for the dispatch with which the manuscript was prepared by the typing staff.

This research was supported in part by a grant from the National Science Foundation (GP-2721) and from the National Aeronautics and Space Aduinistration (NSG 201-62). 
ABSTRACT

ACKNOWLEDGEMENTS

vii

LIST OF ILLUSTRATIONS

$x$

CHAPTER 1. INTRODUCTION

1.1 Two Dynanic Domains 1

1.2 Constraints on Magnetospheric Motions 2

1.3 Ionospheric Damping of Relative Motion Between the Magnetosphere and the Neutrosphere 5

1.4 Dynamo Action, Collisions, and Currents 11

1.5 Historical Review of the Dynamo Theory of the Sq Magnetic Variation

CHAPTER 2. DYNAMICS AND KINEMATICS OE THE DYNAMO REGION 25

2.1 Reference Frame 25

2.2 Equations of Motion 29

2.3 Magnitude of Terms in the Equation of Motion 32

2.4 Resolution of the Wind into Symmetric and Antisymetric Parts

2.5 Resolution of the Symmetric Component of Dynamo Action into Cument Producing and Non-current Producing Parts

(a) Discussion of $v_{I}$, the non-current producing
velocity field.

(b) Discussion of $v_{2}$, the current producing component of velocity.

(c) Discussion of Maeda's treatment.

CHAPTER 3. EEFECT OF SYMMETRIC DYNAMO ACTION with Symmetric Wind Systems

5

1


TABLE OF CONTENTS (CONT'D)

3.3 The Sq Current System

Page

3.4 The Layer Conductivities

53

55

3.5 Wind Systems, Current, and Electrostatic Fields 69

3.6 Representations, Transformations, and Operators 74

3.7 Undetected Zonal Currents and the Effect of Their Omission on Solutions for the Electrostatic Field 80

CHAPTER 4. EFFECT OF ANTISYMMETRIC WIND SYSTEMS

4.1 Antisymmetric Dynano Action 83

4.2 Cuments Driven by Antisymmetric Dynamo Action $8 \$$

4.3 Magnetic and Dynamic Effects of Antisymmetric Dynamo Action

CHAPTER 5. MOTION OE THE MAGNETOSPHERIC PLASMA

5.1 Motions of the Magnetospheric Plasma Produced by Dynamo Action

5.2 Role of Dynamo Action in Determining the Configuration of the Magnetosphere

APPENDIX A DERIVATION OE THE CONDUCTIVITY OF A PARTIALLY IONIZED GAS, WITH THE EQUATION OF MOTION OF THE NEUTRAL CONSTITUENT INCLUDED

APPENDIX B CONDITIONS FOR THE EXISTENCE OF TURBULENCE AT THE LOWER THRESHOLD OF THE DYNAMO REGION

APPENDIX C THE DIVISION OF A HORIZONTAL IRROTATIONAI WIND INTO CURRENT PRODUCING AND NON-CURRENT PRODUCING PARTS 132

REFERENCES 
Fir. I Vector diagram of the forces on a positively changed component of a slightly ionized gas, as seen in a reference frame in which the neutral component is motionless. The mean velocity of the component is shown by vector $\mathrm{vi}$.

Eig. 2 Unipolar inductor. When a barmagnet $\mathrm{N}-\mathrm{S}$ is rotated around its axis $A A^{\prime}$ a current is obtained in a fixed circuit connecting the axis with a sliding contact at C. Erom Alfvén (1950).

Fig. 3 Geometry for the comparison of the relative magnitudes of currents along the field line to the equatorial plane and currents transverse to the magnetic field in the ionosphere under antisymmetric dynamo action. The resistance of the inner tube from the ionosphere to the equatorial plane (Rube) is compared to that of the ionosphere between the inner and outer tube $\left(R_{\text {disc }}\right)$.

Fig. 4 The resistance of a magnetic flux tube having a square meter cross section at the ionosphere. The resistance between the 100, 110, 120 and $130 \mathrm{~km}$. levels and the equatorial plane is plotted as a function of colatitude. Fig. 4 a is for a model appropriate for noon time conditions, $4 b$ for twilight conditions, and $4 c$ for midnight conditions.

Fig. 5 Geometry for the estimate of the displacement of conjugate points caused by the distorting effect of an electric current from one hemisphere to the other along the polar cap field lines.

Fig. 6 Intersection of magnetospheric equipotential sunfaces with the ionosphere. The equipotential surfaces represent the electric field obtained by combining the electrostatic field produced by tidal motions responsible for the production of the Sq magnetic variation (Maeda, 1955) with that associated with the daily corotation of the magnetospheric plasma about the earth.

Fig. 7 Isointensity contours for trapped electrons with energies greater than $40 \mathrm{kev}$. Local time is plotted azimuthally and invariant latitude $(\Lambda)$ is plotted radially. The intensity of trapped radiation falls off rapidly with increased latitude, suggesting that the boundary of the corotating (inner) portion of the magnetosphere occurs near these contouns. From Frank, Van Allen and Craven (1964). 
Fig. 8 Relative positions of the boundaries of the regions in which $280 \mathrm{kev}$ particles, $40 \mathrm{kev}$ particles, and thermal particles are trapped in the magnetosphere. It has been assumed that the limit is imposed on the nightward side where the corotating region has been contracted as the result of day-night asymmetry in the magnetospheric electrostatic field.

Fig. A The complex conductivity (admittance) of the ionosphere in the direction of a transverse electric field which varies sinusoidally. The imaginary part of the conductivity arises from the tendency of the neutral component of gas to be accelerated by collisions with the ionized components. Fig. Al shows the real (R) and imaginary (I) conductivities for applied electric fields with 10 and 100 second periods; Fig. A2, for 6 and 8 hour periods and Fig. A3, for 12 and 24 hour periods. 


\section{CHAPTER 1}

\section{INTRODUCTION}

\subsection{Two Dynamic Domains}

From the dynamic point of view, the earth's atmosphere may be divided into two domains. The Eirst, called the neutrosphere, comprises the neutral atmosphere from ground level to about $100 \mathrm{~km}$. It is the region where the motions of the atmosphere are governed by the ordinary hydrodynamic equations employed in meteorology. The second extends upwards from about $150 \mathrm{~km}$ to include most of the region in space that is affected by the presence of the earth, a volume that has been estimated to be of greater onder than two thousand times that of the solid earth itself. In this domain the dynamic equations are dominated by the electromagnetic terms, and indeed it has been given the name 'magnetosphore' by Gold (2959) just because the region is so much characterized by the presence of the earth's magnetic field.

The range of altitudes separating the two domains is a region of transition. Here, as will later be shown, the equation of motion must include electromagnetic tems along with the hydrodynamic terms, causing the dymamics of this particular region to be more difficult to understand than for either the magnetosphere or the neutrosphere. This is the region of accomodation between the magnetically governed motions of the magnetosphere and the less restricted motions of the neutrosphere. Such accommodation, associated with a boundary condition for each domain, can be expected to involve a transfer of momentum from one domain to the othen through the transition region. The momentun transfer, when viewed 
microscopically, comes about through the accelenation of changed particles by the electric fields and the subsequent collisions between the neutral particles and electrically charged particles co-existing in the transirion pegion. In section 1.4 we shall vee that macroscopically, the transfer of momentum from one layer to another may be considered in the form of a Lorentz Force $\underline{E}$.

$$
\underline{E}=\underline{J} \times \underline{B}=
$$

acting on the partially ionized gas in a particular layer. Because of the relatively small mass of the magnetosphere, such a force will quickly accelerate the magnetospheric material to the velooity of the neutral atmosphere insofar as such motion is permitted by the hydromagnetic equations of motion.

\subsection{Constraints on Magnetospheric Motions}

The fact that certain classes of motion are not permitted in the magnetosphere is manifested by the continued existence of the $\mathrm{Sq}$ current system, which we will find represents the force of the neutral atmosphere acting against the constraints placed on magnetospheric motion by the magnetic field. It is worthwhile considering for a moment the nature of the constraints.

Because of the high conductivity in the magnetosphere, the magnetic field can be said to be frozen into the plasma. This can be simply stated to mean that the time variations of the magnetic field and the motions of the plasma must take place in such a way that the total magnetic flux remains constant through a surface defined by the particles, regardless of how the surface is deformed and translated in the course of the 
fluid's motion. In some applications, such as to gaseous nebulae and the interplanetary magnetic field, this idea is interpreted to mean that the magnetic field is carried about with the motions of the highly conducting plasma. This greatly simplifies many of the discussions by permitting the time variation of the magnetic field and the motion of the plasma to be treated togethes. In our discussion, however, the magnetic field is found to be also frozen into another nearby, but thus far unmentioned dynamic domain, the solid earth. Because of the latter's proximity, rigjdity, and high conductivity, the time variations of the magnetic field in the transition region between the magnetosphere and the neutrosphere are small enough to be treated as perturbations. Because the magnetic field is, to good approximation, held constant by the solid earth, the motions of the magnetospheric plasma are restricted to a class which can take place without producing a redistribution of the magnetic field. Specifically, if the motions are considered to be horizontal, it will be shown in Chapter 2 that the nagnetospheric velocity just above the transition region must be represented by the two dinensional vector field

$$
\underline{v}_{M}=\frac{2}{-\infty} \times \frac{1}{B_{n}} \nabla S
$$

where $I_{n}$ is the unit radial vector, $B_{p}$ is the radial component of the magnetic field and $S$ is a scalar Field, the electrostatic potential. This class of motions is closely related to the non-uniform isorotation first discussed by Ferraro (1937) and the more general class of motions

$$
\underline{v}_{E}=\frac{\underline{E} \times \underline{B}}{B^{2}}
$$


in which no magnetic work is done, as discussed by Gold (1959). Insofar as we are able to assume the earth's magnetic field to be relatively unpenturbed during the motion, a condition which may not prevail at great distances where the magnetic field becomes small, the motions must take place in such a way that all the plasma along a particular magnetic field line moves togethen to simultaneously occupy the positions of other magnetic field lines. Since we will be generally concenned only with steady state situations we will indeed be able to consider this last to be the case. If, however, non-steady states are considered, the plasma along a given field line will continue to occupy common field lines in the course of their motion, but now the field lines must also be considered to move about.

The two conditions just discussed, that requiring the horizontal motion $\underline{Y}_{M}$ of the magnetospheric plasma to obey equation (2.1) and the other requiring all the plesma along a field line to convect as a whole to the position of other field lines, constitute the earlien mentioned constraints on the motion of the magnetospheric material. We find there are two situations in which the forces exerted by the neutrosphere will be directed against these constraints. Finstly, any force tending to produce a magnetospheric motion of the class

$$
\underline{v}_{M}^{\prime}=-\frac{2}{B_{n}} \nabla \underline{y}
$$

acts against the constraint imposed by the difficulty of redistributing the magnetic field in the proximity of the highly conducting solid earth, Secondly, forces oppositely directed in the two hemispheres will act against the constraint that requires all the plasma along a field line 
to move concordantly from one field line to another. Forces of both these kinds occur in the transition region and manifest themselves by producing perturbations of the magnetic field of the kind usually designated $\mathrm{Sq}$. We will find that magnetic perturbations that can be represented by symmetric cumsent systems (i.e., current systems which are mirror images in the equatorial plane; for further discussion see section 2.4) arise from forces acting against the first constraint, while perturbations that are represented by antisymmetric current systems arise from forces acting against the second, on concordant motion, constraint.

\subsection{Ionospheric Damping of Relative Motion Between the Magnetosphere and the Neutrosphere}

A force acting against the constraints on nagnetospheric motion can arise in yet another way. If in some manner a force can be exerted directly on the magnetospheric plasma, it will be transmitted to the transition region, and subsequently to the neutrosphere. Obviously if the force trads to produce a motion requiring a redistribution of the magnotic flux over the earth, it acts against the first constraint. However, even in the case where the resulting motion of the magnetosphere would be of the class permitted from the point of view of magnetic flus distribution, the ionized material in the transition region will be prevented from reaching a velocity concondant with that of the magnetospheric plasma because of numerous collisions with the neutral particles, which because of thein relatively great number and correspondingly large aggregate mass, do not readily accelerate to the velocity of the magnetosphere. Said otherwise, motion having its origin in the magnetosphere will be damped in the transition region, and consequently exists only with the continued action of a driving force. This may be illustrated, following cole (1963) as follows. 
Suppose the ionosphere is initially at rest and the magnetosphere is moving with velocity $\underline{v}_{E}$ given by

$$
-\nabla S+\underline{V}_{E} \times \underline{B}=0
$$

and that no extemal forces are applied to the system. The eleotric field $E=-\nabla S$ accompanying the magnetospheric motion is transmitted along the highly conductive field lines to the transition region where it produces an electric current of density 3. The current gives rise to Joule heating of the transition region and to an acceleration by the Lorentz force $\mathrm{J} \times \underline{\mathrm{B}}$, both effects tending to deplete the kinetic energy of the magnetospheric plasma. An energy equation describing this effect may be written

$\frac{d}{d t} \int \frac{1}{2} p_{M} v_{E}^{2} d V_{M}=-\frac{d}{d t} \int \frac{1}{2} p_{T} v_{T}^{2} d V_{T}-\int \sigma_{I}\left|-\nabla S+V_{T} \times B\right|^{2} d V_{T}$

where $p$ is the gas density, $d V$ is an element of volume, $\sigma_{1}$ is the Pedersen conductivity in the transition region, and the subscripts $M$ and $I$ refer to the magnetosphere and transition region (although $\underline{v}_{E}$ is written rather than $\left.\mathrm{V}_{\mathrm{M}}\right)$. The three integrals represent the kinetic enengy of the magnetosphere, that of the transition region, and the Joule dissipation in the transition region, respectively. The joule dissipation tem is similar to friction in its chanacter, causing the relative motion between the magnetosphere and the transition region to tend imreversibly to zero unless a driving force is maintained on one os the other.

The lange mass of the transition region places an upper limit on $v_{T}$, for suppose that all the kinetic energy of the magnetospheric material 
is transferred to the transition region. Then

$$
\frac{1}{2} M_{T} \bar{v}_{T}^{2} * \frac{1}{2} \int \rho_{M} v_{E}^{2} d V_{M}
$$

where $\bar{v}_{T}$ is the mean velocity of the transition region, $M_{T}$ is the mass of a meter square column of the transition region, and the integral on the right is to be taken over the volume of a tube of magnetic flux with one square meter cross section at the transition region. Express $v_{E}$ as $v_{E O} k\left(r / r_{0}\right)$ where $v_{E O}$ is the magnitude of the magnetospheric plasma velocity just above the transition region, 2 is the geocentric distance of a volume element, $r_{0}$ the earth's radius, and $k$ is a function of position along the Field line, which for middle and low latitude field lines (latitudes less than $60^{\circ}$ ) varies slowly from unity near the ionosphere to less than 4 (twice the tangent of the latitude where the field line intersects the ionosphere) at the field line's intersection of the equatorial plane. We may then write

$$
\frac{1}{2} M_{T} \vec{v}_{T}^{2} \approx \frac{1}{2} v_{E O}^{2} \int \rho_{M} k^{2}\left(r / s_{0}\right)^{2} d v_{M}
$$

Since the mass density $\rho_{M}$ decreases outward much more rapidly than $\left(r / n_{0}\right)^{-2}$, the equation may be changed to an inequality by replacing $\rho_{M} k^{2}\left(r / r_{0}\right)^{2}$ by $P_{\text {MO }}$, the mass density just above the transition region. With this we have

$$
\frac{1}{2} M_{T} \nabla_{T}^{2}<\frac{1}{2} v_{E O}^{2} p_{M O} v_{M}
$$

or

$$
\bar{v}_{T}<\left(\frac{\rho_{M O} v_{M}}{M_{T}}\right)^{\frac{1}{2}} \quad v_{E O}
$$


At $60^{\circ}$ latitude (the upper limit at which this angument should be applied), the volume of a flux tube of meter square cross section at the transition layen is about $10^{10} \mathrm{~m}^{3}$. The density of ionized material in the lower magnetosphere is about $10^{11}$ ions $/ \mathrm{m}^{3}$, and we will take the atomic weight to be 16. This gives

$$
\bar{v}_{T}<10^{-1} v_{E O}
$$

or

$$
\left|\underline{V}_{-T} \times \underline{B}\right|<10^{-1}|\nabla S|
$$

allowing us to neglect the smallen term in the energy equation (3.2). Writing

$$
|\nabla S|=v_{E O} B
$$

equation (3.2) may be written as an inequality

$$
\frac{d}{d t} \int \frac{1}{2} a_{N} v_{E}^{2} d v_{M}<-\int \sigma_{I} v_{E O}^{2} B^{2} d v_{T}
$$

if we consider only the energy loss associated with Joule dissipation. This may be rewritten

$$
\frac{d}{d t} \int \frac{1}{2} p_{M} v_{E}^{2} d v_{M}<-\Sigma_{I} v_{E O}^{2} B^{2}
$$

or

$$
\frac{d}{d t} \int \frac{1}{2} \rho_{M} v_{E}^{2} d v_{M}<-\left(2 \varepsilon_{I} B^{2} \int \frac{1}{2} \rho_{M} v_{E}^{2} d v_{M}\right) / M_{M}
$$

where $M_{M} \equiv \int \rho_{M} k^{2}\left(r / r_{0}\right)^{2} d V_{M}$ and use has been made of the equality of the right hand sides of $(3.3)$ and $(3.4)$. 
The integral is taken over a tube of magnetic flux of square meter cross section at the transition region, and $\Sigma_{I}$ is the Pedersen conductivity integrated through the transition region. Thus, the kinetic energy of the magnetosphere must decay more rapidly than does a quantity I which decays according to the equation

or

$$
\begin{aligned}
& \frac{d T}{d t}=-\left(2 \Sigma_{I} B^{2} / \mathbb{M}_{M}\right) T \\
& T=T_{0} e^{-\frac{t}{\tau}}
\end{aligned}
$$

where

$$
\tau \equiv \frac{M_{1}}{2 \Sigma_{1} B^{2}}
$$

An upper limit for this can be amived at by placing an upper limit on $M_{M}$ as was done earlier.

$$
M_{M}=\int \rho_{M} k^{2}\left(n / r_{0}\right)^{2} d V_{M}<p_{M O} v_{M}
$$

$\Sigma_{1}$ is given by Spreiter and Briggs (1961) as greater than $5 \times 10^{4}$ mhos. Thus, with $B=5 \times 10^{-5}$ weber $/ m^{2}$ we find $\tau$ has an upper limit of $10^{-1}$ sec. The time constant we have obtained is an upper limit for the time which it takes the notion of the magnetospheric material to be damped in the transition region. It is apparent that a continued driving force must be present if motions of magnetospheric origin are to account for phenomena of greater duration than this. The effect of the damping will be continued either for the duration of the existence of the driving force, or until tho seutrosphere is accelerated to a motion consistent with that of the magnetosphere. It is unlikely that the latter condition would be achieved in a reasonable anount of time, if it is indeed at all possible. 
Forces acting directly on the magnetospheric plasma can arise in a number of ways. Gold (1959) has suggested a kind of hydromagnetic "buoyant" force arising when the energy density in the magnetosphere decreases too rapidly with distance outward. The idea is analogous to that of the conveotive instability which exists in a neutral atmosphere when the temporature has a superadiabatic lapse rate. Piddington (1960) and Axford and Hines (1961) have suggested anothen kind of force that could give rise to magnetosphenic circulation. They argue that a viscous-like interaction takes place between the outward streaming solar plasma and the magnetospheric material, causing the latten to be driven in the anti-solar direction. In order that such motion not produce a major redistribution of the magnetic field, a return flow within the magnetosphere was postulated. Axfond and Hines hoped to explain a wide range of geophysical phenomena by means of a general magnetospheric convection driven in this way. Another force acting directly on the magnetosphere, the only one whose existence is at all well established, is that arising from the impact of the solar plasma on the magnetospheric boundary. This produces distortion of the geomagnetic field until a configuration is achieved in which the plasma pressure is balanced by the magnetic pressure of the distonted earth's field. Such a distortion producing force was oniginally suggested by Chapman and Eerraro (1931) to account for the first phase of geomagnetic storms. Later Parker (1960) suggested that solar plasma is always streaming away from the sun, producing a continuing distontion of the earth's field. In orden to maintain the magnetosphere in its position in space the force of the solar wind must be countered by an equal and opposite force exerted on the field in the geosphere and in the conducting 
portions of the atmosphere (Piddington, 1963). Although we will not here be specifically concerned with forces having thein origin within the magnetosphere or at its boundary, it will be of interest to have an estimate of the magnitude of this force. On a quiet day the velocity of the solar plasma has been estimated to be $300 \sim 600 \mathrm{~km} / \mathrm{sec}$ and the density is (2u20) $\mathrm{x}$ $10^{7}$ protons $/ \mathrm{m}^{3}$ (see Panker, 1964 for a compilation of observations). This gives rise to a Force on the boundary of $3 \times 10^{-10}$ to $1.2 \times 10^{-8}$ newtons $/ \mathrm{m}^{2}$. Since the cross section offered by the magnetosphere to the solar wind is about 50 times the area of the earth's entire surface, this force must be balanced by an average force near the earth's surface of $1.5 \times 10^{-8}$ to $6 \times 10^{-7}$ newtons $/ \mathrm{m}^{2}$. Eron an active sun, velocities of onder $2000 \mathrm{~km} / \mathrm{sec}$ and densities of perhaps $3 \times 10^{7}$ protons $/ \mathrm{m}^{3}$ may be expected, leading to forces at the earth's surface as lange as $10^{-5}$ newtons $/ \mathrm{m}^{2}$. These may be compared with the forces between the magnetosphere and neutrosphere in the transition region manifested by the $\mathrm{Sq}$ magnetic perturbation which is of order $10^{-6}$ newtons $/ \mathrm{m}^{2}$.

\subsection{Dymamo Action, Collisions, and Cuments}

Earlien we mentioned in passing that the momentum transfer occurring in the transition region could be viewed microscopically as the result of collisions between neutral particles and charged particles, and macroscopically, as being a Lorentz force. In this section we will discuss the relationship between these two points of view. Let us consider for a moment the behavion of an ion and eloctron gas in the presence of a magnetic field and an orthogonal electric field. If the system is examined in a reference frame moving with velocity $\underline{V}_{E}=E \times B / B^{2}$ the electric field is transformed away, and the ions and electrons are found 
to be gyrating about centers fixed in that reference frame. In such case, the electric field produces no current in the plasma. If the electric field is time varying, the preceding argument fails in that a reference frame moving with velocity $v_{E}$ will no longer be inential. The same will be the case if there are spatial variations of $\underline{E}$ and $\underline{B}$ such that a reference frane moving with velocity $\mathrm{v}_{\mathrm{E}}$ undengoes accelerations in the counse of its motion. Let us suppose, however, that a mean velocity $\bar{v}_{E}$, comresponding to the mean electric field $\underline{E}$ can be defined. The time variation may now be expanded in terms of a Fourien series and treated, following Chandrasekhar (1960), by letting $v_{-1}$ be the velocity of particles relative to a frame moving with velocity $\mathrm{v}_{E}$. We have for the equation of motion

$$
m_{i} \underline{B}_{I}+m_{i} \underline{B}_{E}=q_{i}\left(\underline{E}+\underline{v}_{-1} \times \underline{B}+\underline{v}_{E} \times \underline{B}\right)=q_{i} \underline{v}_{I} \times \underline{B} \quad(4.1)
$$

where $\dot{v}$ is the partial derivative of $\mathrm{V}$ with respect to time, taken in the specified reference frame, $q_{i}$ is the change of the particle of species $i$, and $m_{i}$ is the mass of such particles.

We now define the velocity $\underline{-}_{-}$such that

$$
v_{i} \equiv-\frac{m_{i}}{q_{i}} \frac{v_{E} \times \underline{B}}{B^{2}}=\frac{m_{i}}{q_{i} b^{2}} \underline{E}
$$

having taken B to be constant, and write

$$
v_{1}=v_{-2}+v_{i} .
$$


With this equation (4.I) becomes

$$
m_{i} \dot{v}_{-2}+m_{i-i} \dot{\hat{n}}_{i}=q_{i} \underline{v}_{2} \times \underline{B}
$$

If the characteristic frequency $v$ of the electric field is small compared with the Lamor period $\omega_{i}$ and if $\left|v_{E}\right| /\left|\underline{v}_{2}\right|$ is also small, then the second term on the left can be neglected, since

$$
\frac{m_{i}\left|v_{i}\right|}{q_{i}\left|\underline{v}_{2} \times \underline{B}\right|}=\frac{m_{i}^{2}|\ddot{\underline{E}}|}{q_{i}^{2} B^{3} v_{2}}=\left(\frac{v}{\omega_{i}}\right)^{2} \frac{v_{E}}{v_{2}}
$$

With this approximation, we have

$$
m_{i-2}=q_{i} \underline{V}_{-2} \times \underline{B}
$$

which is the equation for the circular motion of the particle. However, an additional drift motion is now superposed on it,

$$
v_{i}=\frac{m_{i}}{a_{i} B^{2}} \dot{\dot{E}} .
$$

which is called the polarization drift. Since the drift is opposite for particles of opposite sign, this will be associated with a current of density

$$
\underline{J}_{p}=\sum_{i} n_{i} a_{i} v_{i}=\frac{P_{p} \underline{E}}{B^{2}}
$$

where $n_{i}$ is the number density of particles of species $i$.

Taking the cross product of this with $B$ we have

$$
\underline{J}_{P} \times \underline{B}=\frac{\rho_{P}}{B^{2}} \frac{\partial}{\partial t}(\underline{E} \times \underline{B})=\rho_{p} \dot{\vec{t}}_{E} \text {. }
$$


Thus, the polarization current may be considered to be associated with a Lorentz force which accelerates the plasma such that it always moves with velocity $\underline{v}_{E}$.

Let us now assume that there is a neutral component of the gas which we consider to be stationary in oun reference frame. As previously, the charged particles will gyrate in the magnetic field about centers that drift with a velocity $E \times B / B^{2}$, but now thein motion will be frequently dismupted by collisions with the neutrals. In the average collision the amount of momentum transferred from the neutral to the charged particle is

$$
m_{i}\left(v_{i}-\underline{v}_{i}\right),
$$

where $V_{n}$ is the velocity of the neutral particles.

If such collisions occur with a frequency $n_{p} \nu_{i n}$ (where $n_{p}$ is the number density of ions and $v_{\text {in }}$ is the collision frequency of an ion with neutral particles) we have for the total effective force between two typical constituents of the gas

$$
\underline{c}_{\text {in }}=n_{p} \nu_{i n} m_{i}\left(\underline{v}_{n}-\underline{v}_{i}\right)
$$

and for the equation of motion of the constituent $i$

$$
p_{i} \dot{\underline{v}}_{i}=\underline{C}_{i n}+q_{i}\left(\underline{v}_{i} \times \underline{B}+\underline{E}\right)
$$

where all collision terms except those with the neutrals are ignored. If the gas is quite lightly ionized two simplifications may be made. Finst, as we have already assumed, collisions between electrons and ions will be unimportant compared to collisions between the charged panticles 
and noutral particles. Second, a kind of quasi-equilibrium may be considered to exist in which the neutral component may be considered almost unaffected by the collisions with the changed particles. Making these simplifications, the changed components can be considered in a kind of equilibrium under the effect of the electric force, $q_{i} E$, the magnetic force, $q_{i} V_{i} \times \underline{B}_{\text {, }}$ and the Eriction-like fonce produced by collisions with the motionless neutrals, $n_{p} v_{i n} m_{-i}$. Since oun reference frame is such that the neutral component has no mean motion, the magnetic force and the collisional force will be orthogonal to one another. Thus, a vector diagram of the forces on the ionized component at equilibrium $\left(p_{i} \dot{V}_{i}=0\right)$ will be a right triangle with the electric force along the hypotenuse (see Fig. 1). The angle which mean velocity vector $\underline{v}_{i}$ makes with the electric field, designated $\theta_{i}$, is given by the relation

$$
\tan \partial_{i}=\frac{q_{i} v_{i} B}{n_{p} v_{i n} v_{i} v_{i}}=\frac{\omega_{i}}{v_{i n}}
$$

where $w_{i}=q_{i} B / n_{p} m_{i}$

The speed $v_{i}$ may be found from the relationship

$$
q_{i} E \sin \theta_{i}=q_{i} v_{i} B
$$

It has a component in the direction of $\mathrm{E}$

$$
\begin{aligned}
v_{i l l} & =(E / B) \sin \theta_{i} \cos \theta_{i} \\
& =\frac{E}{B} \frac{v_{i n} \omega_{i}}{v_{i n}^{2}+\omega_{i}^{2}}
\end{aligned}
$$


Fig. I Vector diagram of the forces on a positively charged component of a slightly ionized gas, as seen in a reference frame in which the neutral component is motionless. The mean velocity of the component is shown by vector $\underline{v i}_{i}$. 


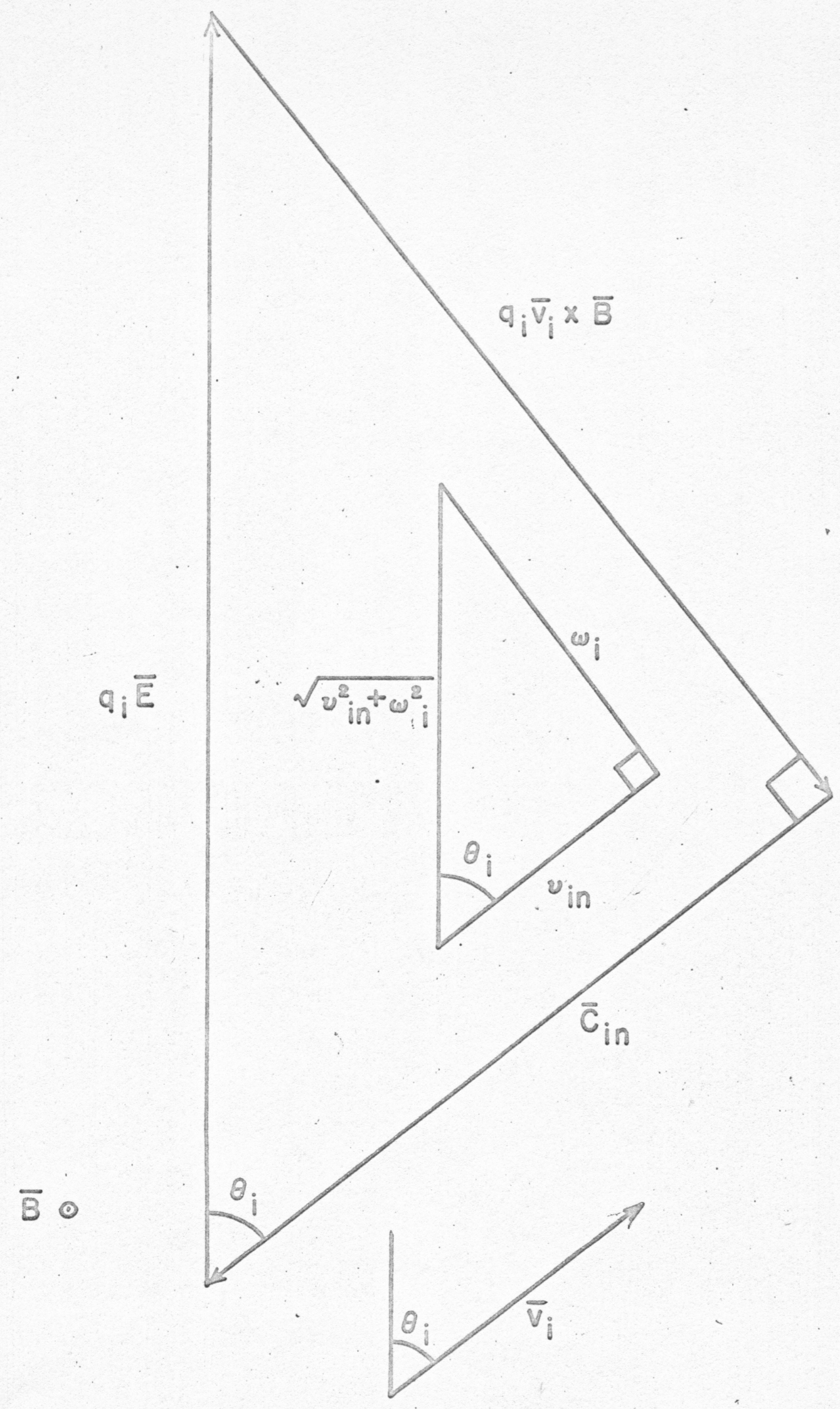


and a component in the direction $\underline{E} \times \underline{B}$

$$
\begin{aligned}
v_{i 1} & =(E / B) \sin ^{2} \theta_{i} \\
& =\frac{E}{B} \frac{\omega_{i}^{2}}{v_{i n}^{2}+\omega_{i}^{2}}
\end{aligned}
$$

The velocity of the electronic component will be similar, the chief difference being that the component $v_{\text {ell }}$ will now be oppositely directed to the electric field, since the gyrofrequency $\omega_{i}$ will become negative with the negative charge on the electron. A discussion of these equations may be found in Chapman and Barcels (1940, Vol, 2) and Martyn (1947). These velocities may now be combined to obtain an expression for the current. Thus

$$
\begin{gathered}
\underline{J}=\operatorname{en}_{p}\left(\underline{v}_{i}-\underline{v}_{e}\right) \\
J_{11}=E e^{2} n_{p}\left[\frac{1}{m_{i}} \frac{v_{i n}}{\left(v_{i n}^{2}+\omega_{i}^{2}\right)}+\frac{1}{m_{e}} \frac{v_{e n}}{\left(v_{e n}^{2}+\omega_{e}^{2}\right)}\right] \\
J_{+}=E e^{2} n_{p}\left[\frac{1}{m_{i}} \frac{\omega_{i}}{\left(v_{i n}^{2}+\omega_{i}^{2}\right)}+\frac{1}{m_{e}} \frac{\omega_{e}}{\left(v_{e n}^{2}+\omega_{e}^{2}\right)}\right]
\end{gathered}
$$

where we have expressed $B$ in terms of the gyrofrequency and mass of each species of particles. In the above and following equations the subscript i. nefers to the positively charged ions. The component of current in the direction of the electric field, $J_{\|}$, is known as the Pedersen current, and that in the direction $B \times E$ (the second, on electron term of $J_{1}$ is negative and largen than the first on ionic term) is known as the Hall current. If a reference frame is employed in which the neutral component 
is in motion, these equations may still be used with E ruplaced by $E+\underline{v}_{-\infty} \times \underline{B}$. Much use will be made of this in later chapters. We will call the vector $\underline{V}_{-n} \times \underline{B}$ the dynamo electromotive force, and $\underline{E}$, which will be found to arise from various accumulations of charge in the magnetosphere, will be expressed in terms of a scalar potential

$$
\underline{E}=-\nabla S
$$

In arriving at the expressions for the Hall and Pedersen currents the somewhat unrealistic assumption was made that the neutral component was always stationary in the reference frame. If we write the equations of motion for the three components, making neither this last assumption, nor the assumption that collisions between electrons and ions are negligible,

$$
\begin{aligned}
& \rho_{n-n} \dot{\underline{v}}_{n}=\underline{C}_{n i}+\underline{C}_{n e} \\
& \rho_{i-i}=\underline{C}_{i n}+\underline{C}_{i e}+q_{i}\left(\underline{E}+\underline{v}_{i} \times \underline{B}\right) \\
& \rho_{e} \dot{v}_{e}=\underline{C}_{n}+\underline{C}_{i}+q_{e}\left(\underline{E}+\underline{v}_{e} \times \underline{B}\right)
\end{aligned}
$$

and take the sum of the three we have

$$
\underline{P} \underline{\underline{V}}=\underline{J} \times \underline{B}
$$

where $p_{\underline{v}}=p_{n-n}+p_{i-i}+p_{e} \underline{v}_{e}$.

Thus, the center of mass of the entire gas may be considered to be accelerated by the Lorentz force $\underline{3} \times \underline{B}$. Insofar as the ionized components may be considered of negligible mass and to be in a quasi-equilibrium 
state, this Lorentz force may be considered to be acting on the neutral component, and in time will indeed produce an appreciable motion of the neutral component. Thus, in treating the conduction of current in a partially ionized gas, either the equations of motions of all the constituents must be included along with a dynamo electromotive force in the current equation, or the motion of the neutral component must be known, as well as the electric field. In the main part of this work the latter course will be followed, since, as will be shown in the following chapter, it is at present impractical to attempt to work with the equations of motion of the dynamo layer. In Appendix A an expression is derived for the current under the steady state application of a sinusoidally varying electric field, including the simplified equations of motion for the three constituents. Since the current will not be in phase with the applied electric field, the conductivities thus derived are analogous to the admittance of a.c. circuit analysis.

We have so far referred to the region separating the neutrosphere and the magnetosphere by the somewhat uninformative narne "transition region". Since the cuments characteristic of the region are primarily driven by the dynamo electromotive force associated with motions of the neutral component of gas, the region has been generally known as the dynamo region or layer. At this point in our development it will be convenient to adopt this term.

1.5 Historical Review of the Dynamo Theory of the Sg Magnetic Variation

Heights above about $90 \mathrm{~km}$ have usually been considered outside the scope of meteorology. However, there was considerable interest in the dynamics of the partially ionized levels above $100 \mathrm{~km}$ even before the 
present interest arising from the recent discoveries of the existence and chanacter of the magnetosphere. This earlier interest was directed toward attempting to establish the origin of the Sq magnetic variation, a daily fluctuation of all three components of the magnetic field having a magnitude of some tens of gammas that persists on days that ane otherwise quite free of magnetic activity. Generally these works followed the suggestion first put forward by Balfour stewart (1882) who, after demonstrating the futility of trying to explain the magnetic fluctuations by some earlier suggestions, such as solar magnetic fields, temperature induced variations in the paramagnetism of oxygen, and themo-electric currents in the earth's crust on atmosphere, advanced the idea that so-called tidal motions of a conducting atmosphere through the earth's magnetic field could produce the currents that give rise to the observed magnetic fluctuations.

The dynamo theory, as Stewart's idea has come to be known, was put into a quantitative form by Schuster (1908) who, after establishing the primarily external oxigin of the magnetic vaniation, demonstrated that the upper atmospheric currents could be explained by convective air motions similar to those observed at ground level. He went on to estimate the total conductivity of the upper atmosphere, and pointed out that, while the conductivity could not be determined with any certainty because of the unknown relationship between the ground level tidal oscillation and that in the dynamo region, it must nevertheless be lange if the tidal oscillations themselves were not to be unexpectedly large.

One gamma is equal to $10^{-9}$ webers per square meter and might be appropriately, but rather awkwardly, referred to as a nanoweber per square meter. 
Chapman $(1913,1918)$ extended Schuster's mathematical theory by employing a more general expression for the variation of conductivity with solar zenith angle. He went on to consider the dynamo theory as applied to the I magnetic variation, that component of the magnetic variation which has a period related to the Iunar day. While the I variation is comparatively small, it offers the advantage of owing its origin to a tide producing force whose character is well known. From this analysis it was determined that the conductivity of the dynamo region must be a factor of ten greaten than that estimated by Schuster. Some difficulties were raised by Redersen (1927), who pointed out that the magnetic field would inhibit currents transverse to itself. Taking this into account, the early calculations of the ionospheric conductivity gave results that were insufficient to meet the requirements of the dynamo theory by more than three orders of magnitude.

Later studies of atmospheric oscillations by Pekeris (1937) and Taylor (1936) reduced this discrepancy by showing that the speed of the tidal motion could be expected to increase with the inverse square root of the ain density. Thus, in the sparce upper atmosphere the oscillations might be a great deal larger than those at the ground. However, the dynamo theory still seemed to be inadequate by a factor of 5 to 10.

The next important step was taken by Hirono (1952), and independently by Baken and Martyn (1954), who showed that a polarization of the medium, arising from the inability of the currents to have a vertical component, could enhance the effective conductivity of the layer sufficiently to allow an explanation of the Sq magnetic variation in terms of the dynamo theory. They also found that the polarization effect would be especially strong near the equator, permitting an explanation of the equatorial 
enhancement of the quiet daily variation. About a year later Fejer (1953) published yet another independent derivation of the enhancement in conductivity arising from the vertical polarization of the layer, and then went on to estimate the magnitude of the ionospheric wind, avoiding certain assumptions made by the earlier workers.

With the dynamo theory now on apparently firm footing, two attempts have recently been made to deduce the wind system in the dynamo region from the observed $\mathrm{Sq}$ magnetic variation. Maeda (1955) solved the differential equations describing the dynamo effect for the wind potential, having made the assumption that the wind responsible for the dynamo action was imotational. Kato (1956) elaborated on this by including with the dynamo equations an equation of motion for the atmosphere in the dynamo region. By assuming that the driving force for the wind could be expressed as the gradient of a scalar, and by taking account of the coriolis force, he was able to deduce both imotational and solenoidal horizontal winds in the dynamo layer. The practicality of this rather attractive approach will be discussed in the next chapter, after the dynamics of the dynamo layer are considered.

Recently, the excitation of atmospheric oscillations has been studied in more detail by Jacchia and Kopal (1952) and most recently by Butler and Small (1963). They examined the gravitationally excited free oscillations of the atmosphere (cf. Pekeris (1936)) for reasonable temperature profiles in the atmosphere and showed that such free oscillations do not exist. Instead, Butler and Small (1963) have shown that observed characteristics of the diumal, semi-diumal and terdiumal oscillations can be explained by themally forced oscillations; they proposed that this is due to a direct absorption of insolation in the ozone layer. Their 
calculation shows that the excited diunnal oscillation is, however, very small in spite of the fact that the diumal component of the absorption is the largest. They attribute this to the smallness of the associated equivalent depths and the lange range in height over which the absorption by ozone is appreciable. Butler and Small (1963) showed that the thermally forced oscillation can explain the major portion of the semi-diunal oscillation of the atmospheric pressure obtained by Simpson (1918), Haurwitz and Sepulveda (1956) and others, without involving the resonance hypothesis. They also claim that the themally excited oscillation has a node a little below $30 \mathrm{~km}$ in height giving the change of phase of $180^{\circ}$ between the atmospheres below and above that level, which is found in the analysis of the quiet day magnetic variations.

Both Maeda (1955) and Kato (1956) showed, however, that the wind system responsible for the Sq variation is more diumal than semi-diumal. This does not necessarily mean, of course, that the semi-diumal component does not exist in the dynamo region. This is discussed funther in Chap$\operatorname{ten} 3$.

To place the present work in the context of the preceding, it is pointed out that most of the earlier works have been directed towand establishing the validity of the dynamo theory, although the latter two might betten be considered applications of the theony to the determination of ionospheric winds. Here we will be primarily interested in estimating the nature of the magnetospheric motion arising from the quite strong coupling of the magnetosphere to the dynamo layer. To undertake such a problem does not immediately take one far beyond the preceding works, since this magnetospheric motion is determined by the electrostatic field arising from the dynamo action, and in each of the earlier papers the 
author has determined such an electrostatic field as a by-product of his calculation of the dynamo layer currents or winds. The new derivation that will appear in Chapter 3 is justified only in that it employs a means. by which the layer conductivities may be represented in a quite general way and in that it makes a somewhat more precise distinction between the current producing component of the wind, and that component which produces no current.

In Chapter 4 anothen refinement to the dynamo theory is made by considering the effect of differences between the winds in the northern and southem hemispheres. While these differences do not influence motions of magnetospheric material, they will produce some distortion of the magnetic field, an effect that could be of importance in interpreting geophysical phenomena at geomagnetically conjugate points. 


\section{CHAPTER 2}

\section{DYNAMICS AND KINEMATICS OF THE DYNAMO REGION}

\section{I Reference Frame}

To avoid some ambiguity in the following where we will be discussing magnetic and electric fields in the vicinity of the rotating earth, it is well to consider at the outset the various reference frames that can be used. The nature of the choice can be well illustrated by means of the unipolar inductor. If a cylindrical bar magnet, which we will assume has symmetry of field as well as of material about its axis, has the leads of a galvanometer connected to it, one at the axis A (see Fig. 2) and the othor by means of a sliding contact to the cylindrical surface at $C$, and if the magnet is rotated, there will be an indication of a current through the galvanometer. This effect can be as well explained in terms of the usual dynamo effect using either a non-rotating reference frame (i.e., one motionless with respect to the galvanometer circuit) on a reference frame which rotates with the bar magnet. From the first point of view, the conducting material of which the bar magnet is composed must be considered to be rotating in its own magnetic field. Thus, if the magnet rotates clockwise about the end where the galvanometer lead is attached, which we will assume to be the end at which the magnetic Field is directed outward, a current is induced toward the center of the bar magnet. From there the current will continue along the external circuit provided by the galvanometer leads. Note that no induction effect takes place in the galvanometer leads as they are motionless in this reference frame. 


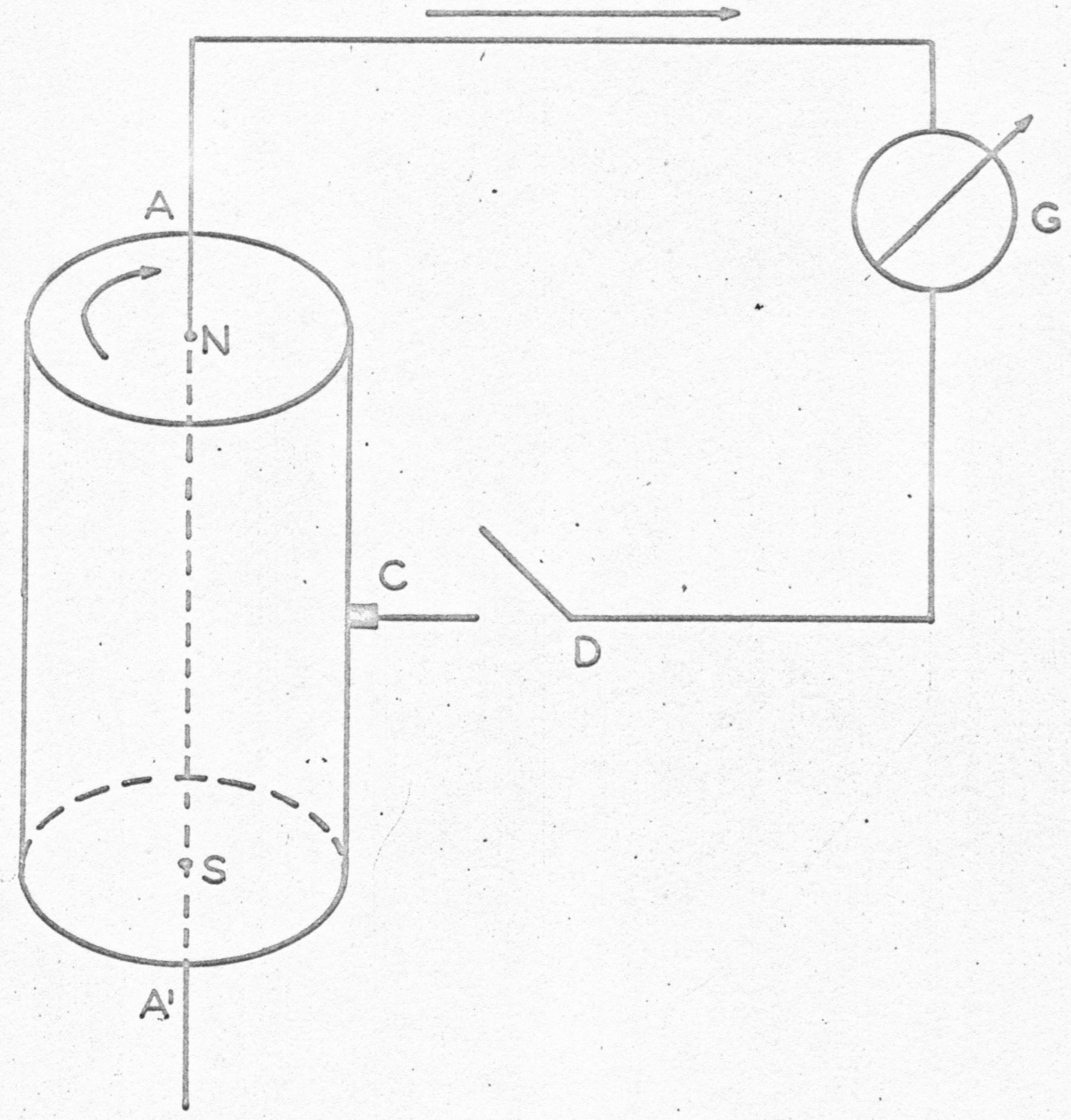

Fig. 2 Unipolar inductor. When a barmagnet $\mathrm{N}-\mathrm{S}$ is rotated around. its axis $A^{\circ}$ a current is obtained in a fixed circuit connecting the axis with a sliding contact at C. From Alfven (1950)。 
From the second point of view, the material of the bar magnet is motionless, so no induction effect takes place there. However, the galvanometer and its leads will now appear to be rotating about the bar magnet's axis in the counterclockwise direction, causing current to be induced in the leads in the direction from the bar magnet's axis to the sliding contact at the cylindrical surface. The material of the bar magnet serves to complete the circuit, but is now considered passive as regards the induction of the cument.

The important point to note is that the behavior of the unipolar inductor can be described in terms of either of these reference frames, or in any other reference frame, without at all having to introduce the concept of "motion of the magnetic field". Indeed, physics has attached no meaning to this phrase.

If there were asymmetry in the magnetic field of the bar magnet just discussed the analysis would be the same. Now, however, when employing the reference frame in which the galvanometer circuit is motionless one finds that there are periodic time variations in the magnetic field, and consequently, comesponding variations in the current.

In the absence of a complete current path, as would be the case if the galvanometer leads were not in contact with the rotating bar magnet, a current would be induced as previously. Now, however, it leads to an accumulation of charge and an electrostatic field which when fully developed will just cancel the induction effect.

In like manner we may use many different reference frames in describing the rotating earth and its magnetosphere. If one is considening effects of the inclined magnetic axis and the anomalies in the field it would be well to employ a system fixed to the solid earth. 
However, if one is content to ignore the smaller scale structure in the Field nean the earth and to idealize the problem further by taking the earth's dipole axis to coincide with the rotational axis, a considerable simplification can be realized in the description of the field at great distances by employing a reference frame that is stationary with respect to the sun-earth line. Thus, the great distortion of the field produced by the solar wind at geocentric distances greater than a few earth radi can be described without large time variations, insofar as the various forces that mold the magnetosphore are in equilibrium.

The motions of the solid earth and of the magnetosphere, from this point of view, will be associated with an electric field $\underline{E}=\underline{V} \times \underline{B}$. As Hines (1959, 1964) has pointed out, the electric field associated with the rotation of the solid earth would by itself tend to produce co-rotation of the magnetosphere by the following process. Poleward earth currents would be induced by the earth's rotation in the geomagnetic field. Such cumpent would continue until there is sufficient charge accumulated at the poles and equator to give rise to an electrostatic field capable of driving equal earth currents equatorward. But such an electrostatic field would exist in the space surrounding the earth as well as within the earth itself, and so would produce a motion of the magnetosphere with velocity $\underline{v}=\underline{E} \times \underline{B} / B^{2}$. However, the motion of the magnetospheric material is more influenced by the motions of the atmosphere in the dynamo region and can be expected to show considerable depanture from the simple rotation dictated by the solid earth. For example, rotation at a greater or lesser rate than the solid earth and meridional components of motion probably exist because of various winds of the atmosphere. 
While we will usually find the reference frame fixed with respect to the sun-eanth line the most convenient, on occasions it will be useful to use a reference frame moving with velocity $\mathrm{V}_{\mathrm{E}}$ such that

$$
\underline{v}_{E}=E \times \underline{B} / B^{2}
$$

In such a reference frame there will be no electric field. If acceleration occurs in the course of such motion, it may be treated either by introducing a time varying electric field, as was done in Chapter 1 while discussing polanization currents, on by introducing a gravity-like force arising from the inertia of the material whose motion is being considered.

\subsection{Equations of Motion}

In this section we examine the equation of motion applicable to the atmosphere in the dynamo layer where the behavior of the gas makes a transition from purely hydrodynamic to purely hydromagnetic. The equation of motion of a gas in a magnetic field may be written (cf. Chandrasekhar $(1961))$.

$$
\rho \frac{\partial \underline{v}}{\partial t}+\rho(\underline{v} \cdot \nabla) \underline{v}=\nabla \cdot \cdot[T]+\rho \underline{X}+H(\nabla \times \underline{H}) \times \underline{H}
$$

In the first approximation we may consider the earth's magnetic intensity $\mathrm{H}$ to be a dipolar field $\mathrm{H}_{0}$; the terms of non-electromagnetic origin, represented by $\rho \underline{x}$ arise mainly from the earth's gravitational field, which we will represent by $\underline{x}_{0} \cdot[\mathrm{T}]$ is the total stress-tensor and includes viscous as well as pressure terms. Let us then consider the zero order approximation in which a steady state with no gas motions is assumed to exist. Then, since $\nabla \times \mathrm{H}_{0}=0$, the above equation reduces to

$$
\nabla \cdot\left[T_{0}\right]+O \underline{X}_{0}=0
$$


the equation of hydrostatic equilibrium. As a first order approximation, we may introduce the rotation of the earth. Under these circumstances we may expect a velocity $\underline{V}_{-}$and perturbations of the stress-tensor [T:] and perturbations of the magnetic field $H_{1}^{\prime}$, where the primes will here be used to indicate perturbation quantities. These give an equation of motion

$$
\begin{aligned}
& \rho \frac{\partial v_{1}}{\partial t}+\rho\left(\underline{v}_{-1} \cdot \nabla\right) \underline{v}_{-1}=\nabla \cdot\left[T_{0}\right]+\nabla \cdot\left[r_{1}^{\prime}\right]+o \underline{X} \\
& +\mu\left(\nabla \times \frac{H_{-1}^{\prime}}{2}\right) \times H_{0}+\mu\left(\nabla \times H_{-1}^{\prime}\right) \times \frac{H_{-}^{\prime}}{1}
\end{aligned}
$$

Applying the equation of hydrostatic equilibrium, and neglecting the term containing the square of $\mathrm{H}_{-1}^{\prime}$, we have in the steady state

$$
p\left(\underline{v}_{1} \cdot \nabla\right)_{-1}=\nabla \cdot\left[T_{1}^{\prime}\right]+\underline{J}_{1} \times \underline{B}_{0}
$$

where we have applied the electromagnetic equations

$$
\begin{aligned}
& \nabla \times H_{-1}^{\prime}=H_{-1} \\
& \underline{B}_{0}=H_{-0}
\end{aligned}
$$

In the case of uniform rotation, the term on the left of $(2.4)$ becomes $-r \Omega^{2} \sin \theta\left(I_{n} \sin \theta+I_{\theta} \cos \theta\right)$ where $\Omega$ is the angular velocity of the earth and $\theta$ is the colatitude. This is the centripetal force that must be applied to the rotating atmosphere. In the neutral atmosphere this is provided by a perturbation pressure field associated with the earth's oblateness. In the ionized portion of the atmosphere the Lorentz force would be capable of contributing to it as well. However, it is unlikely that the magnetic field could long support such a sustained 
force. The diffusion of the plasma through the magnetic field would eventually lead to a redistribution of the ionized atmosphere in which the pressure gradients and centripetal force would be in balance. Thus, in the steady state:

$$
\underline{I}_{2} \times \underline{B}_{0}=0
$$

and

$$
-r \Omega^{2} \sin \theta\left(I_{n} \sin \theta+\frac{3}{-} \cos \theta\right)=\nabla \cdot\left[T_{1}^{\prime}\right]
$$

At greater geocentric distances, where the magnetic field is considered to be retaining the earth's outer atmosphere the Lorentz force must be considered to provide the centripetal force, and possibly a reaction to some pressure tems as well.

We here adopt the custom of the meteorologists and include the centripetal force with the gravitational force $\underline{x}_{0}$ to provide an effective gravity which we denote $x_{-1}$. Pressure perturbations will then be measured from those of rotational equilibrium $\left[T_{1}\right]$ where

$$
\nabla \cdot\left[T_{1}\right]+\Delta X_{1}=0
$$

and the magnetic field will be assumed unperturbed by rotation, as implied by equation 2.6 .

We may now make the next higher approximation in which we may expect a stress-tensor $\left[\mathrm{T}_{2}\right]$, a velocity $\mathrm{V}_{2}$, and a magnetic field $\mathrm{H}_{-2}$ which may be represented in terms of penturbations from the rotational equilibrium values 


$$
\begin{aligned}
& {\left[\mathrm{r}_{2}\right]=\left[\mathrm{T}_{1}\right]+\left[\mathrm{T}_{2}^{\prime}\right]} \\
& \underline{\mathrm{v}}_{2}=\mathrm{v}_{-1}+\frac{\mathrm{v}_{-}^{\prime}}{\mathrm{f}} \\
& \mathrm{H}_{2}=\mathrm{H}_{\mathrm{O}}+\mathrm{H}_{-2}^{\prime}
\end{aligned}
$$

Assuming a steady state, and throwing out all second powers of $\mathrm{v}_{-2}^{\prime}$ and $\mathrm{H}_{2}^{\prime}$, we have

$\rho\left(\underline{v}_{2} \cdot \nabla\right) \underline{v}_{2}^{\prime}+\rho\left(\underline{v}_{2}^{\prime} \cdot \nabla\right) \underline{v}_{I}=\nabla \cdot\left[\mathrm{F}_{2}^{\prime}\right]+\rho \underline{x}_{2}^{\prime}+\mu\left(\nabla \times H_{-2}^{\prime}\right) \times \underline{H}_{0}$

where the driving force for the perturbation may be considered to be in the perturbation stress-tensor, arising from the daily solar heating, and in a perturbation gravity field $x_{-2}^{\prime}$. The terms on the left may be writren

$\rho \Omega \frac{\partial}{\partial \lambda} v_{-2}^{\prime}+2 \rho \Omega \cos \theta\left(I_{n} x v_{-h}\right)-I_{-r} 2 \rho v_{\lambda} \Omega \sin \theta+I_{\lambda} 2 \rho v r \Omega \sin \theta$

and compared with the terms appearing in the equation of motion as obsenved in a rotating reference frame. The finst term is then associated with the usual inertial terms, the second with the coniolis force, the third with a vertical coriolis term which is usually neglected, being small compared to gravity, and the fourth is also usually neglected by assuming the motion to be horizontal.

\subsection{Magnitude of Terms in the Equation of Motion}

The linearized equation of motion obtained at the end of the last section is sufficiently general to be applied to the motions of the dynamo layer. We may now consider the order of magnitude of the various terms to see which of them must be considered in any calculations. The 


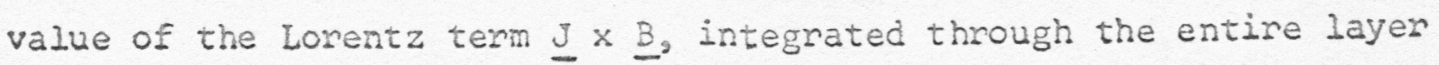
may be calculated with considerable assurance and so may be used as a reference to which the other fonces may be compared. The magnitude of the $\mathrm{Sq}$ magnetic variation, about 30 gammas, can be attributed to a current of about 0.02 amperes/m distributed through the entire dynamo layer. Multiplying this by the magnetic induction of $5 \times 10^{-5}$ webers $/ \mathrm{m}^{2}$ we arrive at a Lorentz force of $10^{-6}$ newtons/meter sq column.

The inertial tems may be next considered, starting with the coriolis terms, $20 \Omega \cos \theta\left(\underline{1}_{n} \times \underline{v}_{h}\right)$. Tidel winds of order $50 \mathrm{~m} / \mathrm{sec}$ in the dynamo region (Kochanski, 1964) have been indicated by sodium cloud release experiments. Taking a middle latitude and a total mass of a meter square column above $100 \mathrm{~km}$ of $3 \times 10^{-3} \mathrm{~kg}$ we find an inertial force of $1.5 \times 10^{-5}$ newtons $/ m^{2}$ column. The finst term of $(2.11)$ of the form $\rho \Omega \frac{\partial}{\partial \lambda} v_{-2}^{\prime}$ may be as great as $2 \times 10^{-6}$ newtons $/ \mathrm{m}^{2}$ for a $50 \mathrm{~m} / \mathrm{sec}$ semi-diurnal wind and half as much for a diurnal wind of that magnitude.

Passing over the perturbation gravity term, the stress tensor includes pressure tems and terms expressing viscous stress. The viscous stress will arise either from the usual process of molecular viscosity or from eddy viscosity if turbulence is present, which is the case between 90 and $100 \mathrm{~km}$. We may obtain an estimate of the viscous force exerted on a meter square column of the dynamo region by considering the viscous stress exerted on a horizontal meter square surface just below the dynamo layer. If we place such a surface within the turbulent region below $100 \mathrm{~km}$, the coefficient of eddy viscosity applies. From observations by Blamont and de Jager (1961) of the scale length, time and velocity of the eddies, Hines (1963) has estimated a coefficient of eddy viscosity of $10^{-4} \mathrm{~kg} / \mathrm{m}$ sec at $95 \mathrm{~km}$. The $50 \mathrm{~m} / \mathrm{sec}$ large scale winds have been observed to undergo 
changes with height of wavelength $20 \mathrm{~km}$, so a shear of order $1.5 \times 10^{-2} \mathrm{sec}^{-1}$ may be expected, giving an eddy viscous stress of $1.5 \times 10^{-6}$ newtons/square meter which may be taken to be applied to the entire overlying column. If one goes to a greater height, above the upper limit of turbulence, the coefficient of molecular viscosity of $10^{-7} \mathrm{~kg} / \mathrm{m} \mathrm{sec}$ applies. Using the same wind shear a molecular viscous stress of $10^{-5}$ newtons/square meter is obtained. This leads to the question of how to account for such a difference in stress. In part, it may be accounted for by an increase in the wind shear near these heights (Kochanski, 1964) which would make the molecular viscous stress larger by a factor of perhaps 5 . The fact that the atmosphere is becoming ionized at these heights suggests the possibility that Lorentz forces may also play a part in transferring horizontal momentum to overlying regions. The point is discussed rather inconclusively in Appendix B, along with the possibility that such effects could account for the cessation of turbulence at the lower boundary of this region. It is there concluded that the molecular viscosity will be more influential in this regard, unless the conductivity is greater near $100 \mathrm{~km}$ than suggested by the model atmospheres that were used in the estimate.

The pressure gradient terms are a bit more difficult to take into account. Kato (1956) attempted to infor a pressure distribution from the observed Sq magnetic variation by using the dynamo equation combined with a simplified hydrodynamic equation. He was thus able to relate the cument, expressed as a current function (analogous to a stream function) to the pressurelike term he assumed to be drivirg the system. Using his results and assuming the dynamo action takes place near $110 \mathrm{~km}$ (since one must employ a mass density in onder to make use of the kinematic pressure he derives) the expected force on a meter square column is found to be $2.5 \times 10^{-6}$ newtons. 
The significance of this number lies in the fact that it is not large compared to the other terms we have already discussed. This suggests that the viscous and Lorentz force terms are not negligible as Kato assumes. The viscous term is perhaps the critical one, for it may be a means by which the dynamo circulation is driven by a solenoidal force (of form $\nabla x$ a) as well as the $\nabla p$ force assumed to exist exclusive of others by Kato. In any case, we may conclude that the pressure term is centainly not lange compared to the other terms in the equation of motion.

\subsection{Resolution of the Wind into Symmetric and Antisymmetric Pants}

Earlien treatments of the dynamo theory have neglected the fact that the dynamo layers in the two hemispheres are joined by highly conducting field Iines. As suggested by Dougherty (1963), it is probably a better approximation to regard the northem and southem dynamo regions as being joined by perfectly conducting wires rather than not being connected at a11. In later sections we verify this last statement; finst we outline the manner in which this problem is here treated.

Consider an operaton $R$ which when applied to $\underline{a}(r, \theta, \lambda)$, a vector field expressed in spherical coondinates (1) changes the sign of the $\theta$ component and (2) replaces the unit vectors with those appropriate to position $(r, \pi-\theta, \lambda)$. Thus

$R \underline{a}(r, \theta, \lambda)=R\left[a_{r}(r, \theta, \lambda) I_{-}(r, \theta, \lambda)+a_{\theta}(r, \theta, \lambda) I_{\theta}(r, \theta, \lambda)+a_{\lambda}(r, \theta, \lambda)\right.$ $\left.\frac{3}{=}(r, \theta, \lambda)\right]$ $\equiv a_{r}(r, \theta, \lambda) I_{-r}(r, \pi-\theta, \lambda)-a_{\theta}(r, \theta, \lambda) I_{0}(r, \pi-\theta, \lambda)+a_{\lambda}(r, \theta, \lambda) \underline{1}_{\lambda}(r, \pi-\theta, \lambda)$ 
Two successive applications of the $R$ operator retum a vector to its initial value, so the $R$ operator is its own inverse,

$$
\underline{a}=R^{-1} R \underline{a}=R R \underline{a}
$$

Any general vector field expressed in spherical coordinates may be written:

$\underline{a}(r, \theta, \lambda)=\frac{1}{2}[a(r, \theta, \lambda)+\underline{R}(r, \pi-\theta, \lambda)]+\frac{1}{2}[\underline{a}(r, \theta, \lambda)-\operatorname{Ra}(r, \pi-\theta, \lambda)]$ (4.3)

The first term on the right may be called the symmetric component of $a(r, \theta, \lambda)$ which we shazl denote $a_{s}(r, \theta, \lambda)$ and the second term, the antisymmetric, denoted $a_{a}(r, \theta, \lambda)$. The names symetric and antisymmetric arise from the property

$$
\begin{aligned}
& \underline{a}_{s}(r, \theta, \lambda)=R \underline{a}_{s}(r, \pi-\theta, \lambda) \\
& \underline{a}_{a}(r, \theta, \lambda)=-R a_{a}(r, \pi-\theta, \lambda) .
\end{aligned}
$$

The symmetry is such that if the space in which a symmetric vector field of this kind is represented were bisected at the plane $\theta=\pi / 2$ by a mirror, the appearance of the system would be the same.

We may define symmetric and antisymmetric scalar functions $g_{s}$ and $g_{\text {a }}$ such that

$$
\begin{aligned}
& g_{s}(r, \theta, \lambda)=g_{s}(r, \pi-\theta, \lambda) \\
& g_{a}(r, \theta, \lambda)=-g_{a}(r, \pi-\theta, \lambda)
\end{aligned}
$$

The gradient of a symmetric scalar function is a symmetric vector, and that of an antisymmetric scalar function is an antisymmetric vector. 
This is obviously true for the $r$ and $\lambda$ components. That the $\theta$ component also assumes the right sign follows if one remembers that a symmetric function as here defined is one that is symetric with respect to $\theta$ about the equatorial plane. The $\theta$ component of $\nabla g_{S}$ is $\frac{1}{r} \frac{\partial g_{S}}{\partial \theta}$ which will be antisymmetric about the equatorial plane. Thus

$$
\nabla g_{S}(r, \theta, \lambda)=R \nabla g_{S}(r, \pi-\theta, \lambda)
$$

A similar argument applies for the antisymmetric case.

The divergence of a symmetric vector field is a symmetric scalar, and of an antisymmetric vector field, an antisymetric scalar. Again, the $r$ and $\lambda$ components are easily understood, so we examine only the term arising from the $\theta$ component of the vector field, $\frac{I}{r \sin \theta} \frac{\partial}{\partial \theta}\left(v_{\theta} \sin \theta\right)$. Multiplication or division by $\sin \theta$, a symmetric scalar, does not influence the symmetry properties, but taking the derivative with respect to $\theta$ reverses them, as is required to verify the stated property. We may thus write

$$
\begin{aligned}
\nabla g_{s}(r, \theta, \lambda) & =R \nabla g_{s}(r, \pi-\theta, \lambda) \\
\nabla g_{a}(r, \theta, \lambda) & =-R \nabla g_{a}(r, \pi-\theta, \lambda) \\
\nabla \cdot \underline{a}_{s}(r, \theta, \lambda) & =\nabla \cdot \underline{a}_{s}(r, \pi-\theta, \lambda) \\
\nabla \cdot \underline{a}_{a}(r, \theta, \lambda) & =-\nabla \cdot a_{a}(r, \pi-\theta, \lambda)
\end{aligned}
$$

The following properties of cross-products among symetric and antisymmetric vectors will be useful:

$$
\begin{array}{lll}
a_{a} \times b_{a} & \text { antisymmetric } \\
a_{s} \times b_{s}- & \text { antisymmetric }
\end{array}
$$




$$
\begin{array}{lll}
a_{s} \times \frac{b}{a}- & \text { symmetric } \\
a_{a} \times b_{-}-\text {symmetric } & (4.7 c)
\end{array}
$$

We prove the second case, all others being the sane except for some changes in sign.

$$
\begin{aligned}
& \underline{a}_{s}(r, \theta, \lambda) \times \underline{b}_{s}(r, \theta, \lambda)=R_{-}(r, \pi-\theta, \lambda) \times \mathbb{R b}_{s}(r, \pi-\theta, \lambda)
\end{aligned}
$$

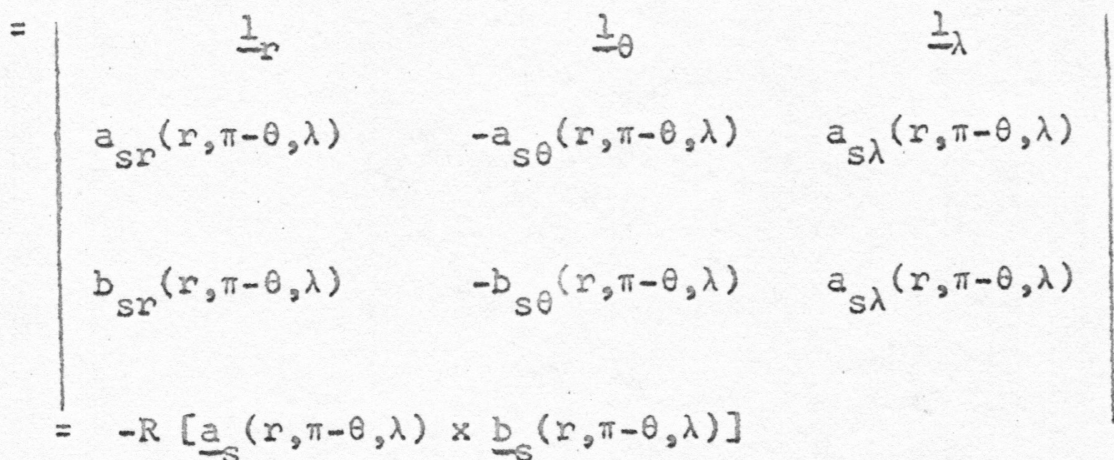

A two dimensional solenoidal vector field $\subseteq$ defined on a shell of constant $r$ may conveniently be described by a stream function $s(\theta, \lambda)$ using the relationship

$$
\underline{c}=\underline{1} \times \nabla s
$$

Since $I_{-n}$ is a symmetric vector, $c$ is symmetric if $s$ is antisymmetric and antisymmetric if $s$ is symmetric.

The preceding may be usefully applied to the dynamo problem where the effects of conductivity along the field line are to be considered. Let the velocity field describing the wind system in the dynamo layen be represented by a vector field $\underline{\mathrm{v}}$. This may be divided into a symmetric and antisymmetric component, as we have just discussed. The vector field representing the geomagnetic field, $\underline{B}$, here assumed to be dipolar, is antisymmetric. Consequently, the dynano electromotive force associated 
with the symmetric component of the wind, $\underline{v}_{S} \times \underline{B}$, is symmetric, while that associated with the antisymmetric wind component is antisymmetric. Assuming the conductivities to be symetric, the dynamo-driven Pedersen current as well as the dynamo driven Hall current will have the same symmetry properties as the wind that drives them. This is easily seen in the case of the Pedersen current. As regards the Hall current, which is of the form $\frac{\sigma_{2}}{B} \underline{B} \times(\underline{v} \times \underline{B})$, one need only note that neither taking the vector product with an antisymmetric vector (B) non multiplication by a symmetric scalar produces a change in symmetry.

From (4.6) it follows that the divergence of the dynamo driven current will be symmetric on antisymetric depending on whether the current itself is respectively symmetric on antisymetric, and will give rise to electrostatic fields with comesponding symetry.

At the equatorial plane, the $\theta$ component of symmetric vector fields is zero, since

$$
a_{s \theta}(r, \theta, \lambda)=-a_{s} \theta(r, \pi-\theta, \lambda)
$$

while the $r$ and $\lambda$ components may take finite values. For antisymmetric vector fields, the $\theta$ component may take finite values while the $r$ and $\lambda$ components must be zero at the equatorial plane.

It follows that $v_{-}$will lead to no current along the field lines, since such a current would have to be entirely in the $\theta$ direction at the equatorial plane, but $\underline{v}_{s}$ could lead to finite electrostatic fields transverse to the magnetic field at the equatorial plane (in the $\lambda$ and $r$ directions). The electrical state of eithen hemisphere would not be altered in the least if the field lines were severed at the equatorial plane and 
left open circuited. Consequently, symmetric dynamo action may be treated by considering only one hemisphere and assuming the field lines simply end at the equatorial plane with no connection between them. Antisymetric dynamo action will lead to finite currents along the field lines but with no electrostatic field transverse to the magnetic field (in the $r$ and $\lambda$ directions) at the equatorial plane. Here the situation would be in no way changed by introducing a perfectly conducting equatorial plane, allowing us to treat the problem of antisymmetric dynamo action by again considering only one hemisphere, but now having all the field lines terminate at the equatorial plane in a short circuit.

Thus, if we employ linearized equations of motion, and a linear dynamo equation, we may divide the problem into two parts, one treating symmetric fields and the other antisymetric fields. Each of these need be considered only in one hemisphere, the effect of the other hemisphere being taken care of by the application of the appropriate boundary condition at the equatorial plane.

2.5 Resolution of the Symmetric Component of Dynamo Action into Current Producing and Non-current Producing Parts

Gold (1959) noted that there are certain types of motion of the magnetospheric plasma which involve no magnetic work, or said otherwise, that bring about no redistribution of the magnetic field. In the dynamo layer and just above, such redistributions of the magnetic field are quite negligible, so the magnetospheric plasma may here be considered to be constrained to such a class of motions. The magnetosphere will tend to folLow the motions of the dynamo region because of the strong coupling between the two. However, it will only follow such motions insofar as they are in accondance with the constraints imposed by the magnetic field distribution. 
It is useful to divide the motion of the dynamo region into two components, one that the magnetosphere can follow and the other that tends to move the magnetosphere in opposition to the constraints.

Antisymmetrie motions in the dynamo layer cannot be followed by the magnetosphere because all the material on the same field line at any instant must move togethen from one field line to another, a condition which we have been considering a second constraint. Here the symmetric component of motion will be considered exclusively.

The class of motion discussed by Gold (1959) may be expressed

$$
\underline{v}_{E}=\frac{-\nabla S_{1} \times \underline{B}}{B^{2}}
$$

where $S_{1}$, the electrostatic potential, is constant along each field line. Thus, $\nabla S_{1}$, is orthogonal to $\underline{B}$ and its radial $(r)$ and latitudinal $(\theta)$ components are thus related:

$$
\left\{\nabla S_{1}\right\}_{\theta}=-\left(\nabla S_{1}\right)_{r} \tan x=-\left(\nabla S_{1}\right)_{r} \frac{B_{r}}{B_{\theta}}
$$

Here $x$ is the dip angle of the magnetic field and $\left(\nabla S_{1}\right)_{\theta}$ is the latitudinal (southward) component of the gradient of $S_{1}$. Using (5.2) and expressing $B$ as $\left(I_{n} B+{ }_{n} B_{\theta}\right),(5.1)$ may be written

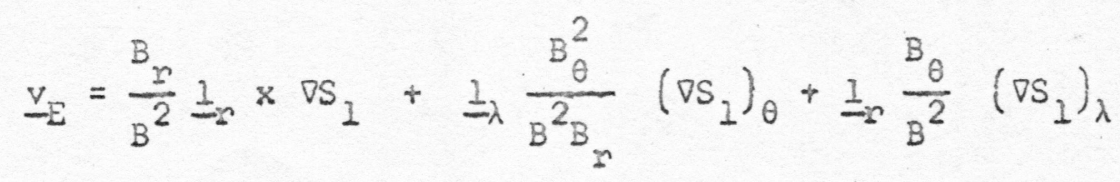

The constraints that have been discussed place no restriction on motion along the magnetic field, and the above equation gives only the component 
of motion perpendicular to the field. Since the motion in the dynamo region is assumed to be horizontal, a motion along the field, $\underline{v}_{\|}$, will be added to the above, having a radial component $-\frac{{ }_{B}}{B^{2}}\left(\nabla S_{2}\right)_{\lambda}$. Its component in the $\theta$ direction is then $-\frac{B_{\theta}^{2}}{B_{r} B^{2}}\left(\nabla S_{1}\right)_{\lambda}$, allowing us to write

$$
\begin{gathered}
\underline{v}+\underline{v}_{11}=\frac{B_{r}}{B^{2}} \underline{I}_{r} \times \nabla S_{1}+\underline{I}_{\lambda} \frac{B_{\theta}^{2}}{B^{2} B_{r}}\left(\nabla S_{1}\right)_{\theta}-\frac{1}{\theta} \frac{B_{\theta}^{2}}{B_{r} B^{2}}\left(\nabla S_{1}\right)_{\lambda} \\
=\frac{1}{B_{r}} t_{r} \times \nabla S_{1}
\end{gathered}
$$

If we define $\underline{v}_{-1} \equiv \underline{v}_{E}+\underline{v}_{11}$ and rewrite $(5.4)$ as

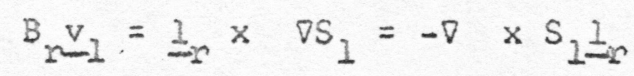

we find that $S_{1}$ may be considered a stream function for the vector field $\mathrm{B}_{\mathrm{r}} \mathrm{v}_{1}$, which implies that

$$
\nabla \cdot B_{2} \underline{v}_{2}=0
$$

the condition upon which the constraint is based.

A vector field limited to a finite region of space may be uniquely separated into solenoidal and irrotational components. Such a division may be made here, so the portion of the horizontal velocity in the dynamo layer not expressible by a velocity field of the form $v_{1}=\left(1 / B_{r}\right) \nabla \times S_{1}{ }_{r}$ may be expressed as

$$
v_{2}=-\frac{1}{B_{r}} \nabla \psi
$$


where $\psi$ is a scalar potential. Generaily

$$
\nabla \cdot B_{r-2} \nabla^{2} \psi \neq 0
$$

so such motion will be a kind which the magnetosphere is unable to follow. Consequently, a force of constraint in the form of a Lorentz force will be exerted on the gas of the dynamo layer. That this is indeed the case for a velocity field of the form $\underline{V}_{2}$ as defined in (5.6), and not for one of the form $\mathrm{v}_{-1}$ defined in $(5.4)$, will now be demonstrated.

(a) Discussion of $\underline{v}_{1}$, the non-current producing velocity field. A wind of form $V_{-1}$ will produce a dynamo electromotive force

$$
\underline{v}_{1} \times B=\nabla S_{1}
$$

which will drive a layer integrated current $I_{D}$, where

$$
I_{D}=[\Sigma] \cdot \nabla S_{1}
$$

Here $[\varepsilon]$ is a layer conductivity tensor and the subscript $D$ on the current vector signifies that it is the component of current driven directly by dynamo action. In the case of symmetric dynamo action, the layen conductivities derived by Hirono (1952) and Baker and Martyn (1954) are appropriate, as will be discussed in Chapter 3 , and only the horizontal components of $(5.9)$ need be considered. The tensor conductivity has the important property that the elements on the diagonal are positive. These are associated with the component of current in the direction of the applied electric field, the Pedersen current. 
As a consequence of the divergence of the dynamo driven current (5.9), an electrostatic field will be established which for the moment will be represented as the negative gradient of a potential $S_{1}^{\prime}$. It will now be shown that in the steady state $S_{1}^{\prime}$ will differ from $S_{1}$ by no more than a constant. In the steady state the divergence of the total cumrent is zero, so the electrostatic field driven current $I_{E}=-[\Sigma] \cdot \nabla S_{I}^{\text {' }}$ must be such that

$$
0=\nabla \cdot\left(I_{D}+I_{\Sigma}\right)=\nabla \cdot\left([\Sigma] \cdot \nabla\left(S_{1}-S_{1}^{1}\right)\right)
$$

Consider the function $S_{1}-S_{1}^{1}$. If it is not simply a constant it may be represented in the form of a contour map on the hemisphere, and there must be some point, line on area at which $s_{1}-s_{1}^{\prime}$ takes a maximum value. There, the gradient $\nabla\left(S_{1}-S_{1}^{\prime}\right)$ must be inward over the contoun at the boundary of the maximum region. But then, since [ $\Sigma]$ has positive elements on its diagonal, the current

$$
I_{D}+I_{E}=[\Sigma] \cdot \nabla\left(S_{1}-S_{1}^{i}\right)
$$

will have an inward component along this entire boundary, and will be convergent. Thus, $S_{1}$ and $S_{1}^{2}$ can differ at most by a constant. Since $S_{1}$ and $S_{1}^{\prime}$ enter the equations only through its gradient, they may henceforth be used interchangeably.

(b) Discussion of $\underline{v}_{2}$, the current producing component of velocity. A wind represented by the velocity field $\underline{v}_{2}$ given in equation $(5.6)$ will give rise to a horizontal electromotive force

$$
\left(\underline{v}_{2} \times \underline{B}_{h}=I_{2} \times \nabla \psi=-\nabla \times \underline{\Psi}\right.
$$


which will drive a current

$$
\underline{I}_{D}=-[\Sigma] \cdot \nabla \times \underline{\Psi}_{r}
$$

In the steady state the divergence of $I_{D}$ must be just cancelled by the divergence of the current $I_{E}$ driven by the electrostatic field $-\nabla S_{2}$. Thus

$$
0=\nabla \cdot\left(\underline{I}_{D}+\underline{I}_{E}\right)=-\nabla \cdot[\varepsilon] \cdot\left(\nabla \times \psi_{2} \underline{I}_{r}+\nabla S_{2}\right)
$$

The quantity $\nabla \times \psi_{-r}+\nabla S_{2}$ cannot be zero except in the trivial case where both terms are zero, so the current $I_{D}+I_{E}$ will generally be non-zero, and a Lorentz force will act on the gas of the dynamo layer as it moves with a velocity of form $v_{2}$.

(c) Discussion of Maeda's treatment.

Having divided the velocity field of the dynamo layer into a current producing part $\underline{v}_{2}$ and one $\underline{v}_{1}$ which produces no current, it is interesting to examine earlier treatments of the dynamo theory of the Sq magnetic variation. For example, Maeda (1955) assumed as did earlier workers, that the wind system responsible for $\mathrm{Sq}$ could be expressed as a vector field of the forn

$$
\underline{v}=-\nabla \phi
$$

where $\phi$ will have only horizontal gradients. If this is multiplied by $B_{r}$ the resulting vector field

$$
-B_{r} \nabla \phi=-B_{p} \cos \theta \nabla \phi
$$


(where $B_{p}$ is the magnetic field at the pole) may be expressed as a function of two scalar fields $S_{1}$ and $\downarrow$ such that

$$
-B_{p} \cos \theta \nabla \phi=-\nabla \times S_{1}=-\nabla \psi-\nabla \psi
$$

Differential equations for $S_{I}$ and $\psi$ are derived in Appendix $C$. Here we note that the curl of the left side of $(5.17)$ may be written

$$
\begin{aligned}
-B_{p} \nabla \times(\nabla \phi \cos \theta) & =B_{p} \nabla \phi \times \nabla \cos \theta \\
& =\underline{1}^{B} B_{p} r^{-2} \frac{\partial \phi}{\partial \lambda}
\end{aligned}
$$

which, not being zero in general, indicates that Maeda's velocity field cannot be expressed in terms of the current producing velocity field $\underline{v}_{2}$ alone. Rathen, it includes a component of wind which does not participate in the production of current in the dymamo layer, and whose existence cannot be deduced from the observations of the Sq magnetic variation. However, Maeda's solution based on the assumption that the wind is of the form $-\nabla \phi$ is no less valid than the results of a calculation of the wind based on the assumption that it is of the form $\mathrm{v}_{2}=-\nabla \psi / \mathrm{B}_{\mathrm{r}}$. In each case there is an additional component of the wind about which the Sq magnetic variation gives no infomation, and in each case this unknown component of wind is of the form $v_{-1}=-B_{r}^{-1} \nabla \times S_{1}=r^{2}$, but not of the form $v_{\text {unknown }}=$ $-\nabla \times R I$. If one wishes to separate the wind velocity field into irrotational and solenoidal pants one must consider both to contribute to the current system, and must somehow manage to divide the observed current system between the two before being able to carry out a solution. 
The above discussion indicates that the dynamo equations alone cannot be uniquely solved for either the solenoidal or irrotational component of the dynamo layer wind. All one can hope to obtain is a solution for the irrotational component of the vector field $B_{n} v$, unless the equations of motion for the dynamo layer are included in the derivation to provide a relationship between the irrotational and solenoidal components of $\underline{v}$. 
CHAPTER 3

EFFECT OE SYMMETRIC DYNAMO ACTION

\subsection{Motion of the Magnetospheric Plasma Associated with Symmetric} Wind Systems

It was noted in section 2.5 that symmetric dynamo action gives rise to electrostatic fields transverse to the magnetic field. As originally pointed out by Martyn (1953), along with such electrostatic fields there is established a motion of the magnetospheric plasma of the form $\mathrm{v}_{E}=$ - $\nabla S \times B / B^{2}$. In addition, there may be wotion along the field lines, such motion not being directly affected by the electromagnetic forces that are dominant in the magnetosphere. Since symmetric dymamo action gives rise to no currents in the direction of the magnetic field, the field lines may be regarded as equipotential lines that lie on surfaces along which the magnetospheric plasma nust flow. Such motions are of the class that is required by what has here been referred to as the constraints on magnetospheric motions. The purpose of the present section is to link the magnetospheric motions to the dynamo layer wind systems that drive them. This may most conveniently be done by separating the dynamo layer wind into current producing and non-current producing components.

There is probably no need to discuss at very great length the magnetospheric motion associated with the component of the dynamo layen wind which produces no current, for this component was originally introduced as being consistent with the motions that the magnetospheric plasma can undergo. However, two things are perhaps worth reiterating. First, if such a component of wind does exist in the dynamo layer, in the steady state the magnetospheric material will also have such a component of motion. This is implied 
in the derivation of section 2.5 , which indicates that the electrostatic potential produced by the non-current producing component of wind may be considered to be equal to the stream function for the vector field $\mathrm{B}_{\mathrm{V}} \mathrm{I}$. The second point is that neither the magnitude, configuration, nor even the existence of such a component of motion can be inferred from the Sq magnetic variation without introducing the equations of motion for the dynamo layer, much as Kato (1956) has done. However, the viscous stress transferred by (and probably responsible for) the turbulent region below the dynamo region must be included if such an approach is attempted. This will require either determination of the shear field (on better yet, the stress field) of the turbulent region over the globe, which will serve as the boundary conditions for a rather elaborate form of the Navier-Stokes equation. The altemative approach and perhaps the more reasonable one, is to devise techniques for measuring the electric fields in the magnetosphere or what is the same thing, the motions of the magnetospheric plasma above about $150 \mathrm{~km}$. The part of the total magnetospheric motion arising from the current producing pontion of the dynamo layer wind may be detemined by means of the conventional dynamo theory applied to the Sq magnetic variation, and extracted from the total. The remaining velocity field will be the non-cument producing component of motion in the dynamo layer.

3.2 Motion of the Magnetosphere Associated with the Current Producing Component of Wind in the Dynamo Region

The current producing portion of the dynamo layer wind, $\underline{v}_{2}=$ - $\left(1 / B_{n}\right) \nabla \psi$ has here been referred to as the component of dynamo layer motion which the magnetosphere is unable to follow. However, such a 
wind does produce a convection of the magnetospheric plasma. Indeed, the effect here is a considerable one.

As there are no currents along the field lines, the layen conductivities derived by Hirono (1952) and independently by Baken and Martyn (1954) may be employed, obviating the need to consider the vertical component of the dynamo electromotive force. These layer conductivities are in the form of a tensor

$$
[\Sigma]=\left(\begin{array}{cc}
\Sigma_{\theta \theta} & \Sigma_{\theta \lambda} \\
-\Sigma_{\theta \lambda} & \Sigma_{\lambda \lambda}
\end{array}\right)
$$

where the diagonal elements are associated with the Pedersen conductivity and the off diagonal elements are associated with the hall conductivity, all of which are effectively enhanced by (1) a ventical polarization field arising from the inability of charge to flow vertically away from the layen and (2) the inclination of the magnetic field, which makes the conductivity in the north-south direction somewhat greater than would be the case if the current were strictly transverse to the magnetic field. The layer conductivities are discussed in greater detail in section 4 of this chapter. Here we need only note that only the horizontal electric fields need be considered to determine the current and that in the dynamo layer the off diagonal conductivities are usually larger than those represented by the diagonal elements (an exception to this occurs near the equator). The dyramo electromotive force produced by a wind $\underline{v}_{-2}=-\left(1 / B_{r}\right) \nabla \psi$ will have horizontal components

$$
\underline{v}_{2} \times \underline{I}_{n} B_{n}=\frac{1}{2} \times \nabla \psi
$$


and will drive a Hall current, integrated through the layer,

$$
\begin{aligned}
\underline{I}_{D H} & =\Sigma_{\theta \lambda}\left(I_{-\infty} \times \nabla \psi\right) \times I_{-} \\
& =\div \varepsilon_{\theta \lambda} \nabla \psi
\end{aligned}
$$

since $\psi$ has only a horizontal gradient. If the conductivities were uniform over the globe $I_{D H}$ would be an imotational vector field and would have divergence $\Sigma_{\theta \lambda} \nabla^{2} \psi ;$ with non-uniform conductivity $I_{D H}$ will have some solenoidal component as well, but will still show considerable divergence. In similan fashion, the Pedersen cument would be strictly solenoidal were the diagonal elements of the tenson equal to one another and unifom over the globe, but in a more realistic model they too are likely to show divergence. However, for simplicity of the present discussion let us suppose that all these idealizations hold, as would be the case if the magnetic field were vertical and the ionosphere uniform over the entire globe. This would cause the diagonal elements $\Sigma_{\theta \theta}$ and $\Sigma_{\lambda \lambda}$ to equal the Pedensen conductivity $\Sigma_{P}$ and the magnitude of the off-diagonal elements $\Sigma_{\theta \lambda}$ to equal the Hall conductivity $\Sigma_{H}$. Then the divergence of the dynamo driven cument is $\Sigma_{H} \nabla^{2} \psi$. In the steady state there will be an electrostatic field $-\nabla S_{2}$ driving a cument with equal and opposite divergence. In our region of finite extent this implies that the irrotational component of cument driven by the electrostatic field will be equal and opposite to the imotational component of the dynamo driven current.

Under the present idealizations the irrotational part of the current driven by the electrostatic field will be that associated with the Pedersen conductivity, so we must have

$$
\Sigma_{\mathrm{P}} \nabla S_{2}=\Sigma_{H} \nabla \psi
$$


The electrostatic field $-\nabla S_{2}$ will be associated with magnetospheric plasma motions

$$
\underline{v}_{E}=-\nabla S_{2} \times \underline{B} / B^{2}=-\left(\Sigma_{H} / \Sigma_{p}\right) \nabla \psi \times \underline{B} / B^{2} .
$$

This equation deals with motion onthogonal to the field only. If a component of motion along the field is included so that the motion is honizontal (as might be approximately the case in the dynamo layer and lower magnetosphere) we have

$$
\begin{aligned}
\underline{v}_{E h}=\left(1 / B_{n}\right) \cdot I_{n} \times \nabla S_{2} & =+\left(\Sigma_{H} / \Sigma_{p}\right) I_{n} \times \frac{1}{B_{n}} \nabla \psi \\
& =-\left(\Sigma_{H} / \Sigma_{p}\right) \underline{I}_{n} \times \underline{v}_{-2}
\end{aligned}
$$

But since $\Sigma_{\theta \lambda}>\Sigma_{p}$ we have

$$
\left|\underline{v}_{E h}\right|>\left|\underline{v}_{2}\right|
$$

This phenomenon has a less exoric analogy in sailing, where on a reach* a boat can move faster than the wind that drives it (although modern racing yachts do not seem capable of such performance; see Batchelon and Davies (1956)). In such circumstances the sail is set so that the sail force has a considerable component in the forward direction. In the steady state the abeam component of the sail force acts against the constraint established by the keel in the water and a steady state velocity is attained when the friction-like resistance between the hull and water equals the forward component of force.

*The tack sailed by a ship with the wind coming just forward of the beam or with the wind directly abeam on abaft the beam. 
In the magnetosphere the "set of the sails" is established by the ratio of the Hall conductivity to the Pedersen conductivity, the greater the ratio the relatively greaten will be the component of Lorentz force in the direction of unconstrained magnetospheric motion, or so to speak, forwand. The steady state is achieved when this force is just balanced by the resistance to magnetospheric motion produced by a friction-1ike drag against the neutral gas component in the dynano region, represented by the Lorentz force of the electrostatic field driven Pedersen current.

The above discussion applies to a rather over-simplified case. The inclusion of the non-unifomity of the conductivities over the globe and the inequality of $\Sigma_{\theta \theta}$ and $\Sigma_{\lambda \lambda}$ will complicate the problem and will modify the pattern of motion established in the magnetosphere. However, the additional complexities will not invalidate the general features of the relationship between magnetospheric motion and dynamo layer winds here discussed.

\subsection{The Sg Current System}

In section 2.5 it was demonstrated that symmetric dynamo layer winds of the form $\underline{v}_{2}=-\frac{1}{B} \nabla \psi$ can produce ionospheric currents, while those of the form $\underline{v}_{-1}=-\frac{1^{2}}{B_{r}} \nabla \times S_{1}=$ cannot, because only the former motion acts to redistribute the earth's magnetic field. Here we continue to discuss the idealized case in which the conductivities are uniform over the globe, and the direct conductivity (Pedersen) is the same in the east-west direction as it is in the north-south, in order to gain insight into the relationship between the fundamental process for the generation of current and the kinematics of the wind system. Essentially the same discussion was presented by Baker (1954) except that he assumed the causative wind to be strictly irrotational. 
In the case of a wind of the form $v_{1}=-\frac{1}{B_{r}} \nabla \times S_{1}{ }_{n}$, as already noted, the horizontal dynamo electromotive force

$$
v_{1} \times I_{2} B_{2}=\nabla S_{1}
$$

is under steady state conditions everywhere cancelled in the dynamo layer by the establishment of an equal and opposite electrostatic field $-\nabla S_{1}$, so that there will be no current. On the other hand dynamo layen winds of the form $\underline{v}_{-2}=-\frac{1}{B_{r}} \nabla \psi$ give rise to a horizontal dynamo electromotive force of the form

$$
\underline{v}_{2} \times I_{-r} B_{r}=-\nabla \times \psi I_{r}
$$

which clearly cannot be cancelled by the irrotational electrostatic field. The dynamo electromotive force will drive a component of current in its own direction

$$
I_{D P}=\sum_{p=1} \times \nabla \psi
$$

and another component orthogonal to itself in the layer

$$
\underline{I}_{D H}=\Sigma_{H} \nabla \psi
$$

Similarly, the electrostatic field $-\nabla S_{2}$ will have components of current in its own direction, and orthogonal to itself

$$
\begin{aligned}
& I_{E P}=-\Sigma_{p} \nabla S_{2} \\
& I_{E H}=\Sigma_{H=P} \times \nabla S_{2}
\end{aligned}
$$


Under our simplifying assumptions $I_{D H}$ and $I_{E P}$ will be entirely irrotational while $I_{D H}$ and $I_{D P}$ are entirely solenoidal. In the steady state we must have no imotational component of the total current, so IEF must be $I_{\text {DH. }}$. Thus

$$
\nabla S_{2}=\frac{\Sigma_{H}}{\Sigma_{P}} \nabla \psi
$$

and the total solenoidal component of current becomes

$$
I_{D P}+I_{E H}=\frac{\Sigma_{P}^{2}+\varepsilon_{H}^{2}}{\Sigma_{P}} I_{-} \times \nabla \psi
$$

Baker and Martyn (1954) have denoted $\left(\Sigma_{\mathrm{p}}^{2}+\Sigma_{H}^{2}\right) / \Sigma_{\mathrm{P}}$ by the symbol $\Sigma_{3}$, and it is sometimes referred to as the cowling conductivity. The important point to observe here is that the two solenoidal components of current, one driven by dynamo action and one driven by the electrostatic field are in the same sense, hence the polarization of the layer serves to augment rather than reduce the solenoidal component of the dynamo driven cursent.

Aside from some modification produced by the non-uniformity of the conductivities over the globe and by the change in the direct conductivity with direction, it is this cument which can be considered responsible for the dominant antisymetric component of the $\mathrm{Sq}$ magnetic variation, where symmetry and antisymmetry in the sense of section 2.4 is here intended.

\subsection{The Layer conductivities}

Recent treatments of the dynamo theory (Hirono, 1952; Baker and Martyn, 1954; Fejer, 1953) have all reduced the problem of dynamo action in a three dimensional region to a two dimensional problem by introducing the concept of a layer conductivity and an effective or mean wind. The usual derivation of the layen conductivity relates the current density 
at any level to the horizontal component of the electric field by means of $\mathrm{Ohm}$ 's law with tenson conductivity and the additional condition that there be no vertical component of current at any level. In order to meet this last condition the vertical component of the electric field is made to assume a value such that it drives a vertical current equal and opposite to that driven by the horizontal components of the field. Since this condition determines the vertical component of the electric field, the horizontal currents driven by this component are also determined. This permits the total horizontal current density to be expressed in terms of the horizontal components of the electric field, the constants of proportionality being the tensor conductivity for an incremental layer. To find the total current through the entire dynamo region, the current density is integrated through height. If it is now assumed that the horizontal electric field is constant with height it may be taken out of the integral, leaving only the conductivity inside. This integral is then the layen conductivity which relates the horizontal electric field, taken to be uniform through the layer, to the total horizontal current in the layer.

The assumption made early in the derivation, that there is no vertical component of cument at any level, seems a difficult one to justify and an impossible one to realize in general. This condition would require, in the steady state when the current must be divergence free, the existence of an electrostatic field $E=-\nabla S$ capable of driving a current system $\mathrm{J}_{S}$ such that

$$
\nabla \cdot \underline{I}_{S}=-\nabla \cdot \underline{J}^{-D}
$$

where $I_{D}$ is the current driven by dynamo action. Simultaneously, the additional condition would have to be met 


$$
J_{n S}=-J_{n D}
$$

where the $r^{\prime}$ s denote radial components.

Except in some special cases, such as that where the current system of the electrostatic field is everywhere equal and opposite to that driven by dynamo action*, it will be impossible to find a scalar function $s$ that simuitaneously satisfies both conditions. Thus, it is preferable to attempt to avoid this assunption altogether.

Hines $(1962,1963)$ has pointed to another shortcoming of this treatment, its disregard of the fact that there may be appreciable wind shear and even reversals in the wind's direction from one level in the dynamo layer to another. Here an attempt will be made to develop apparatus to allow the discussion of the dynamo action of a stratified wind while avoiding the objectionable assumption discussed earlier.

It is natural to reduce the three dimensional dynamo problem to one involving only two dimensions when we attempt only to explain magnetic variations at ground level as the integrated effect of currents at all levels in the dynamo layer. The vertical distribution of the cument density has little influence on the magnetic perturbation, so that it is convenient to deal with the problem in tems of currents, winds, and conductivities integrated over all levels. Going in the reverse direction, as Maeda (1955) and Kato (1956) did, one can hardly expect to deduce more than a single wind velocity field, characterizing the layer as a whole, from the single vector field, the magnetic perturbation at ground level. Then, it is clearly advantageous to discuss dynamo theory in terms of

* This would require a wind uniform with height of the form $\underline{v}_{1}=$ $-\left(I / B_{r}\right) \nabla \times S_{I}=_{r} \cdot$ (see Chapter 2 , section 5) 
integrated quantities, so we here define the layer integrated current I

$$
I(\theta, \lambda) \equiv \int_{r_{0}}^{r} J(r, \theta, \lambda)\left(r / r_{0}\right) d r
$$

where the integration is to be carried over a trapezoidal surface extending from a base of unit length at height $r_{0}$ somewhere below the dynamo layer to one of length $\left(r_{1} / r_{0}\right) \approx I$ at $r_{1}$, a height above the region of dynamo action. The integration results in a two dimensional vector field having three elements on components. The cumrent density J may be expressed

$$
\underline{J}=[\sigma] \cdot(\underline{\nabla} \times \underline{B}-\nabla S)=[\sigma] \cdot \underline{E}
$$

where $[\sigma]$ is the tenson conductivity. In expanded form the above becomes

$$
\underline{J}=\sigma_{0 \sim 0}+\sigma_{1} \underline{E}_{I}+\left(\sigma_{2} / B\right) \underline{B} \times \underline{E}_{1}
$$

where the electric field $E$ has been separated into a component $E_{0}$ along the magnetic field and another, $E_{1}$, orthogonal to it. Thus the layer integrated cument will be a vector sum involving terms typified by

$$
\int_{r_{0}}^{\infty} \sigma_{1}(r, \theta, \lambda) E_{1 \theta}(r, \theta, \lambda) d r
$$

where we approximate $\left(r_{0} / n_{1}\right)$ by one.

It is convenient to express the three dimensional fields $[\sigma], \underline{\mathrm{V}} \times \underline{B}$, and $\nabla S$ in terms of a set of orthogonal functions. For the $\theta$ and $\lambda$ dependence surface hamonics will be employed, while the radial variation will be expressed in tems of a Fourier series. For example, a scalar 
function $F(r, \theta, \lambda)$ may be thus expressed

$F(n, \theta, \lambda)=\sum_{n m j}\left[f_{a}(j, n, m) \cos j K\left(n-n_{0}\right)+f_{b}(j, n, m) \sin j K\left(n-n_{0}\right)\right] e^{i m \lambda_{p} m}(\cos \theta)$

where $k=2 \pi /\left(n_{2}-n_{0}\right)$ and where $f_{a}(j, n, m)$ and $f_{b}(j, n, m)$ are comple. Thus equation (4.7) allows us to represent $F(r, \theta, \lambda)$ as a pair of three dimensional arrays of complex numbers.

If another scalan function $G(r, \theta, \lambda)$ is similarly represented in $(j, n, m)$ space, the representation of the product $F(r, \theta, \lambda) G(r, \theta, \lambda)$ requires a convolution in $(j, n, m)$ space, or said otherwise, requires multiplying two expressions of the form given in (4.7) and the organization of the resulting expression into the specified form to give a representation of $H(r, \theta, \lambda)$ in $(j, n, m)$ space where

$$
H(r, \theta, \lambda) \equiv F(r, \theta, \lambda) \quad G(r, \theta, \lambda)
$$

However, in integrating such products over $r$ we may use the orthogonality of the functions $\cos j k\left(n-r_{0}\right)$ and $\sin j k\left(n-r_{0}\right)$; the integrated product then becomes

$\int_{r_{0}}^{r} F(r, \theta, \lambda) G(r, \theta, \lambda) d r=\sum_{n} \sum_{m} \sum_{n^{\prime} m^{\prime}} h^{i}\left(n, m, n^{\prime}, m^{\prime}\right) e^{i\left(m+m^{\prime}\right) \lambda_{P} m} P_{n}(\cos \theta) P_{n}^{m^{\prime}}(\cos \theta)$

where

$$
\begin{aligned}
& h^{\prime}\left(n, m, n^{\prime}, m^{\prime}\right)\left(r_{1}-n_{0}\right) f_{a}(0, n, m) g_{a}\left(0, n^{\prime}, m^{\prime}\right) \\
& +\frac{1}{2}\left(n_{1}-n_{0}\right) \sum_{j=1}\left[f_{a}(j, n, m) i_{a}\left(j, n^{\prime} m^{\prime}\right)+f_{b}(j, n, m) \varepsilon_{b}\left(j, n^{\prime}, m^{\prime}\right)\right] .
\end{aligned}
$$


However (4.9) may also be written

$$
\begin{aligned}
\int_{0}^{r} F(r, \theta, \lambda) G(r, \theta, \lambda) d r=\left(r_{1}-r_{0}\right) F_{a, 0}(\theta, \lambda) G_{a, 0}(\theta, \lambda) \\
+\frac{1}{2}\left(r_{1}-r_{0}\right) \sum_{j=1}\left[F_{a, j}(\theta, \lambda) G_{a, j}(\theta, \lambda)+F_{b, j}(\theta, \lambda) G_{b, j}(\theta, \lambda)\right]
\end{aligned}
$$

where

$$
F_{a, j}(\theta, \lambda) \equiv \sum_{n} \sum_{m} f_{a}(j, n, m) e^{i m \lambda_{P} m}(\cos \theta)
$$

We here refer to the quantity defined by (4.12) as the $j$ th cosinusoidal component of the radial spectrum of $F(r, \theta, \lambda)$. Similarly, $F_{b, j}(\theta, \lambda)$ defined in the same manner will be the $j$ th sinusoidal component of the radial spectrum of $F(r, \theta, \lambda)$. Each component of the radial spectrum can be corsidered as characteristic of the dynamo layer as a whole, and as such, presents a convenient means by which the discussion of the dynamo theory for a stratified layer can be presented.

An important property of this representation is that the integrated products (4.6) will have magnitude only insofar as $[\sigma(r, \theta, \lambda)]$ and $E(r, \theta, \lambda)$ have the same components in their radial spectra. For example, E may have some appreciable components in its radial spectrum that contribute little to the integral, and thus little to the observable effects of dynamo action simply because the conductivity $[\sigma]$ does not have corresponding components in its radial spectrum. Generally, the Hall conductivity and the Pedersen conductivity will have different radial spectra, and so one might find that the conductivity tensor has, for example, a greater Pedensen than Hall element in cemain parts of its radial spectrum, and greater Hall than Pedersen in other. 
By means of this representation the layer integrated current may be expressed

$$
\begin{aligned}
I(\theta, \lambda) & =\left(r_{1}-r_{0}\right)\left[\sigma_{a, 0}(\theta, \lambda)\right] \cdot E_{a, j}(\theta, \lambda) \\
& +\frac{1}{2}\left(r_{1}-r_{0}\right) \sum_{j=1}\left\{\left[\sigma_{a, j}(\theta, \lambda)\right] \cdot \underline{E}_{a, j}(\theta, \lambda)+\left[\sigma_{b, j}(\theta, \lambda)\right] \cdot E_{b, j}(\theta, \lambda)\right\}
\end{aligned}
$$

and the components of the radial spectrum of the electric field may be expressed in terms of the comesponding components of the radial spectra of the wind velocity and the electrostatic field.

$$
E_{a, j}(\theta, \lambda)=V_{-a, j}(\theta, \lambda) \times \underline{B}(\theta, \lambda)-E_{S a, j}(\theta, \lambda)
$$

where $E_{S}$ is written to indicate that the gradient rather than the scalar potential itself, is to be divided into radial spectral terms, since*

$$
\underline{E}_{S a, j}(\theta, \lambda) \neq-\nabla S a, j(\theta, \lambda)
$$

The current density may be separated into three components corresponding to those of the electric field that drives it. That part of the current driven by the dynamo electromotive force we will call $\mathrm{J}_{-2}$ that driven by the component of the electrostatic field perpendicular to the magnetic field we will call $J_{S}(1)$, and that part driven along the magnetic field lines by the component of electrostatic field in that direction we shall call $\mathrm{J}_{-}(11)$. Because of the relatively high conductivity along the field lines, the component of the electrostatic field in that direction can be much smallen than the electric fields perpendicular to the magnetic

\footnotetext{
"This may be seen by considering the radial component of the electrostatic field. A sinusoidal component of the radial spectrum of $S$ will give rise to a cosinusoidal component in the radial spectrum of the vertical component of $\nabla S$.
} 
Eield while driving cunrents of comparable magnitude. Consequently, in considering curnents nomal to the magnetic field we may, to good approximation, neglect the existence of potential gradients along the field lines and considen the field lines to be equipotentials. Then $-\nabla S(\perp)$, the electrostatic field nomal to the magnetic field lines, will vary negligibly along the field lines as they pass through the dynamo layer. Funthermore, although $-\nabla S(1)$ will vary in the direction nomal to the magnetic field, the anount of such variation along a vertical line as it passes through the dynamo layer will not be very large over most of the globe. The size scale of such variations in the horizontal direction is the same as the horizontal scale of the wind systems from which they arise and the vertical scale of a variation having a horizontal scale $I$ will be $I \tan x$ where $x$ is the dip angle of the magnetic field. The wind systems with which we will be concerned have horizontal scales of order $10^{3} \mathrm{kilo-}$ meters, so the vertical scale of such variations will become as small as the few tens of kilometers thickness of the dynamo layen only within a few degrees of the equator. Thus, over the greater part of the globe the radial spectrum of $-\nabla S(1)$ may be taken to consist of only the zeroth or constant tem, and the portion of the layer integrated current driven by $-\nabla S(1)$ may be written

$$
\begin{aligned}
I_{S}(1) & =\int_{r_{0}}^{r} J_{S}(1)(r, \theta, \lambda) d r \\
& =-\int_{r_{0}}^{r}[\sigma(r, \theta, \lambda)] d r \cdot \nabla S(1)(\theta, \lambda) \\
& =\left(r_{1}-r_{0}\right)[\sigma a, 0(\theta, \lambda)] \cdot E(1)_{S a, 0}(\theta, \lambda)
\end{aligned}
$$


The current density driven directly by the dynamo electromotive force may next be considered. In terms of the wind velocity, the dynamo driven current is

$$
\begin{aligned}
& \underline{J}_{D}=[\sigma(r, \theta, \lambda)] \cdot \underline{v}(r, \theta, \lambda) \times \underline{B}(\theta, \lambda) \\
& =\left\{\sum_{j}\left[\sigma_{a, j}(\theta, \lambda)\right] \cos j k\left(r-r_{0}\right)+\sum_{j}\left[\sigma_{b, j}(\theta, \lambda)\right] \sin j k\left(r-r_{0}\right)\right\} . \\
& \left\{\sum_{j}, \underline{v}_{a, j^{\prime}}(\theta, \lambda) \cos j^{\prime} K\left(r-r_{0}\right)+\sum_{j} \underline{v}_{b, j}(\theta, \lambda) \sin j^{\prime} K\left(r-r_{0}\right)\right\} \times \underline{B}(\theta, \lambda)
\end{aligned}
$$

After multiplication the resulting terms will contain one of the three following factors and may be replaced by a comesponding pain of factors:

$$
(4.28 a)
$$

$\cos j K\left(r-r_{0}\right) \cos j^{\prime} K\left(r-r_{0}\right)=\frac{1}{2} \cos \left(j+j^{\prime}\right) K\left(r-r_{0}\right)+\frac{1}{2} \cos \left(j-j^{\prime}\right) K\left(r-r_{0}\right)$

$(4.18 b)$

$\cos j k\left(r-r_{0}\right) \sin j^{\prime} k\left(r-r_{0}\right)=\frac{1}{2} \sin \left(j+j^{\prime}\right) K\left(r-r_{0}\right)-\frac{1}{2} \sin \left(j-j^{\prime}\right) K\left(r-r_{0}\right)$

$\sin j k\left(r-r_{0}\right) \sin j^{\prime} K\left(r-r_{0}\right)=\frac{1}{2} \cos \left(j-j^{\prime}\right) K\left(r-r_{0}\right)-\frac{1}{2} \cos \left(j+j^{\prime}\right) k\left(r-r_{0}\right)$

If $j=j^{\prime}$ the factors $(4.18 a)$ and $(4.18 c)$ give

$$
\frac{1}{2} \pm \frac{1}{2} \cos 2 j k\left(r-r_{0}\right)
$$

while $(4.18 \mathrm{~b})$ gives

$$
\frac{1}{2} \sin 2 j k\left(r-r_{0}\right)
$$

If $I_{D}$ is integrated through the dynamo layer to obtain the contribution of the dynamo action to the layer integrated current, the terms containing sinusoidal or cosinusoidal factors will contribute nothing, since there is an integer number of cycles in the range of integration. 
In (4.19) only terms with the constant Eactor one-half (on one in the case of $j=j^{\prime}=0$ where $\cos 2 j k\left(r-r_{0}\right)=1$ ) will contribute to the layer integrated current responsible for the $\mathrm{Sq}$ magnetic variation. This is only another way of saying that the functions in terms of which the radial distributions of conductivity and electric field are expanded are orthogonal functions. However, we have here shown that the contributions to the layen integrated current are from the constant terms in the radial spectrum of the current density, and that these may be considered to be distinct current systems, having current density uniform with height in the dynamo layer. While the preceding arguments were made in terms of the dynamo driven portion of the current, quite similar arguments hold for the currents driven by the electrostatic fields. The total layer integrated current may be written

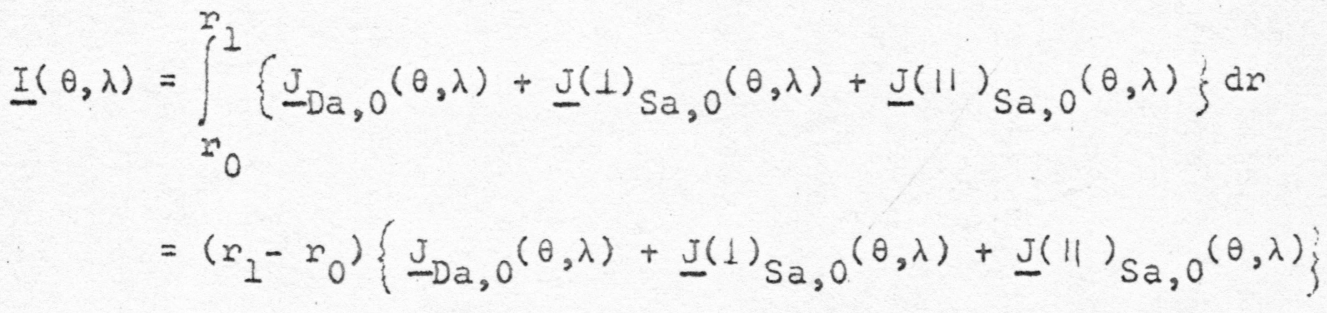

$$
\begin{aligned}
& =\left(r_{1}-r_{0}\right) \underline{J}_{-, 0}(\theta, \lambda)
\end{aligned}
$$

The subscript, a, supplies no information about the zeroth component of the radial spectrum, so a component such as $J_{-D a,}(\theta, \lambda)$ will hereafter be written $J_{D}, 0(\theta, \lambda)$.

It may be shown that the horizontal components of the constant term in the radial spectrum of $\mathrm{J}$ form a current system that may be considered independent of the vertical component of the constant term as well as of all other terms of the radial spectrum. Integrating the quantity $\nabla . \underline{J}$ 
through the dynamo layer in a manner analogous to that used to define the layer integrated current, except that the integration is now carried over a truncated conical volume having a square cross section of unit area at height $r_{0}$ and a square cross section of area $\left(r_{1} / r_{0}\right)^{2}$ at $r_{1}$, we have

$$
\begin{aligned}
\int_{r_{0}}^{r_{1}}\left(r / r_{0}\right)^{2} \nabla \cdot J d r & =\int_{r_{0}}^{r_{1}}\left\{\frac{2}{r^{2}} \frac{\partial}{\partial r}\left(r^{2} J_{r}\right)+\frac{1}{r \sin \theta} \frac{\partial J}{\partial \lambda}+\frac{1}{r \sin \theta} \frac{\partial}{\partial \theta}\left(J_{\theta} \sin \theta\right)\right\}\left(r / r_{0}\right)^{2} d r \\
& =r_{0}^{-2} \int_{r_{0}}^{r_{2}} \frac{\partial}{\partial r}\left(r^{2} J_{r}\right) d r+\nabla \cdot \int_{r_{0}}^{r_{I}}\left(I_{\lambda} J_{\lambda}+I_{\theta} J\right)\left(r / r_{0}\right) d r \\
& =\nabla \cdot I_{n}
\end{aligned}
$$

Here $I_{h}$ is the horizontal part of the layer integrated current and is considered to be at height $r_{0}$, since $r$ is replaced by $r_{0}$ in the divergence operator. The integral involving $J_{r}$ is zero because the current density is zero at $r_{0}$ and $r_{1}$ in the case of symmetric dynamo action. But since in the steady state $\nabla \cdot J=0$ everywhere, the height integrated divergence must likewise be zero, from which it follows, since $\left(r / n_{0}\right)^{2} \approx 1$, that

$$
\nabla \cdot I_{h}=\left(r_{1}-r_{0}\right) \nabla \cdot \underline{I}_{n, 0}=0
$$

Suppose for a moment that $\underline{J}_{0}$ is a transverse current driven by the combined effects of the dynamo electromotive force $\underline{v} \times \underline{B}$ and the transverse electrostatic field $-\nabla S(1)$. Any vertical component of such a current would lead to divengence at the lower $\left(r=r_{0}\right)$ and upper $\left(r=r_{1}\right)$ 
Wwitaing surfaces of the dynamo layer, and the growth of a layer

polarization field. In the steady state the layer polarization would just suffice to drive a current along the field lines having an equal and opposite vertical component to that driven by the transverse electric fields $(\underline{v} \times \underline{B}$ and $-\nabla S(1))$. The transverse current produced by this

polarizarion field would be small and is here neglected, so we may consider the polarization field to be directed along the field lines, denoting it by $-\nabla S(11)$ and the current it drives will be called $J(\|)_{S}, 0$. However, it is most convenient to separate $J(\|)_{S, O}$ into two parts, $I^{\prime}(\|)_{S, O}$ and $I^{\prime \prime}(\| 1)_{S, 0^{\circ}}$ The first is associated with ${\underset{J}{D}, 0}_{0}$ so that

$$
J_{r D, 0}=-J^{2}(11)_{r S, 0}
$$

and the other is similanly associated with $J(1)_{S, 0^{\circ}}$ since $I^{\prime}(11)_{S, O}$ is in the direction of the magnetic field, $(4.24)$ requires that it have a $\theta$ component:

$$
J^{\prime}(\|)_{\theta S, 0}=J^{\prime}(\|)_{n S, 0} \cot x=-J n D, 0 \cot x
$$

and since $\mathrm{J}_{D}$ is normal to the magnetic field

$$
J^{\prime}(11)_{\theta S, 0}=J_{\theta D, 0} \cot ^{2} x
$$

By means of $(4.24)$ and $(4.26)$, the combined current system $\mathrm{J}_{\mathrm{D}, 0}+$ $\left.\mathrm{J}^{\prime}(1)\right)_{S, 0}$ may be written

$$
\vec{J}_{D, 0}+\underline{J}^{\prime}(\|)_{S, O}=\underline{I}_{\lambda} J_{\lambda D, O}+\underline{I}_{\theta}\left(1+\cot ^{2} x\right) J_{\theta D ; 0}
$$


This last equation may be interpreted to indicate an enhancement of the $\theta$ component of $J_{D}$ by the polarization of the dynamo layer; it is a result quite similar to those obtained by earlien workers, the only difference being that we have here neglected transverse currents driven by the vertical polarization of the layer.

Hereafter, we will be concemed only with the layer integrated currents, and so we will use the notation

$$
\underline{I}_{D}=\left(r_{2}-r_{0}\right)\left(\vec{I}_{D, 0}+\underline{J}(11)_{S, 0}\right)
$$

and similarly for the current driven by the electrostatic field

$$
I_{S}=\left(r_{I}-r_{0}\right)\left\{J(1)_{S, 0}+I^{n}(11)_{S, 0}\right\}
$$

where both of these current systems are entirely horizontal.

The condition that the dynamo electromotive force $\underline{V} \times \underline{B}$ and the electrostatic field $-\nabla S(1)$ are both perpendicular to the magnetic field allows the vertical component of the electric field to be eliminated from the equation. When this is done the layer integrated current may be written in terms of the horizontal electric field as

$$
\begin{aligned}
I(\theta, \lambda) & =\left[\Sigma_{a, 0}(\theta, \lambda)\right] \cdot \underline{E}_{h a, j}(\theta, \lambda) \\
& +\frac{1}{2} \sum_{j}\left[\Sigma_{a, j}(\theta, \lambda)\right] \cdot E_{h a, j}(\theta, \lambda)+\frac{1}{2} \sum_{j}\left[\Sigma_{b, j}(\theta, \lambda)\right] \cdot E_{h b, j}(\theta, \lambda)
\end{aligned}
$$

where $E_{h a, j}$ is the horizontal part of $E_{-a, j}(\operatorname{see}(4.14))$ and $\left[\varepsilon_{a, j}(\theta, \lambda)\right]$ is the jth cosinusoidal component of the radial spectrum of the layer conductivity tensor. This may be written 


$$
\left[\Sigma_{a, j}(\theta, \lambda)\right]=\left(\begin{array}{ll}
\frac{\left(r_{1}-r_{0}\right)}{\sin ^{2} x} \sigma_{1 a, j}(\theta, \lambda) & \frac{\left(r_{1}-r_{0}\right)}{\sin x} \sigma_{2 a, j}(\theta, \lambda) \\
\frac{\left(r_{1}-r_{0}\right)}{\sin x} \sigma_{2 a, j}(\theta, \lambda) & \left(r_{1}-r_{0}\right) \sigma_{1 a, j}(\theta, \lambda)
\end{array}\right)
$$

Hore $\sigma_{1 a, j}(\theta, \lambda)$ is the $j$ th cosinusoidal tem in the radial spectrum of the Pedersen conductivity and $\sigma_{2 a, j}(\theta, \lambda)$ is the $j$ th cosinusoidal tem in that of the Hall conductivity. These ane determined by the expressions

$$
\begin{aligned}
& \sigma_{1 a, j}(\theta, \lambda)=\left(r_{1}-r_{0}\right)^{-1} \int_{r_{0}}^{r_{1}} \sigma_{1}(r, \theta, \lambda) d r \\
& \sigma_{l a, j}(\theta, \lambda)=2\left(r_{1}-r_{0}\right)^{-1} \int_{r_{0}}^{r_{1}} \sigma_{1}(r, \theta, \lambda) \cos j k\left(r-r_{0}\right) d r, j \neq 0 \\
& \sigma_{l b, j}(\theta, \lambda)=2\left(r_{1}-r_{0}\right)^{-1} \int_{r_{0}}^{r_{1}} \sigma_{1}(r, \theta, \lambda) \sin j k\left(r-r_{0}\right) d r
\end{aligned}
$$

The layer conductivities derived by the earlier workers correspond to the zeroth or constant tem in the radial spectrum of the layer conductivity. Also the dynamo electromotive force used by them is the constant radial spectral term of the present discussion.

While the techniques of this section provide a means for treating the dynamo action of a stratified wind system, their application, beyond rough estimates of the effectiveness of various terms in the radial spectrum of the wind in producing magnetic perturbations, must await a 
more adequate understanding of the distribution of winds in the dynamo layen. The previous applications of the dynamo theory have attempted either to deduce ionospheric current systems from assumed wind systems, or conversely, to deduce the wind system fron the current systems that produce the observed magnetic perturbations. The latter course is no longen open if one wishes to include the effects of a stratified wind system, since only one wind system can be deduced from the single twodimensional layer current systems that all analysis of the Sq magnetic variation have to date produced. Only if the current system can be separated into components attributable to various terms in the radial spectrum of the wind velocity, on if it can be show that only one radial spectrum term is substantially effective, will there be a system of equations that allows the winds to be deduced.

On the other hand, the dynamo theory, since the time when the anisotropic conductivity of a partially ionized gas was first understood, has been adequate, although perhaps a bit cumbersome, to treat the problem of deducing a dynamo current system from a given distribution of winds and conductivities. All that has been lacking is adequate knowledge of these distributions. Hopefully, with modern rocket sounding techniques, this information will in time become available.

\subsection{Wind Systems, Current and Electrostatic Fields}

If the dynamo layer winds and conductivities are known and have been expressed in terms of their radial spectra, the currents and electrostatic fields produced by wind dynamo action can be computed. From (4.30) the integrated layen current arising directly from dynamo action may be written 


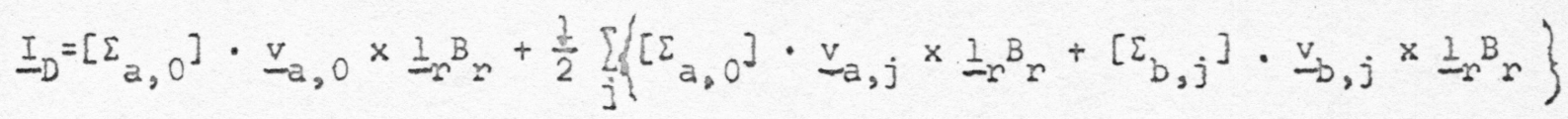

where for conciseness the $\theta$ and $\lambda$ dependence is left understood. In general ID will have divengence, causing the growth of an electrostatic field, $-\nabla S$, until the current Is driven by the electrostatic field has equal and opposite divergence to that of $I_{D}$. At this time the total current, I, will be solenoidal and expressible in terms of a current function T. Thus, we may write

$I=I_{D}+I_{S}=-I_{2} \times \nabla \Gamma$

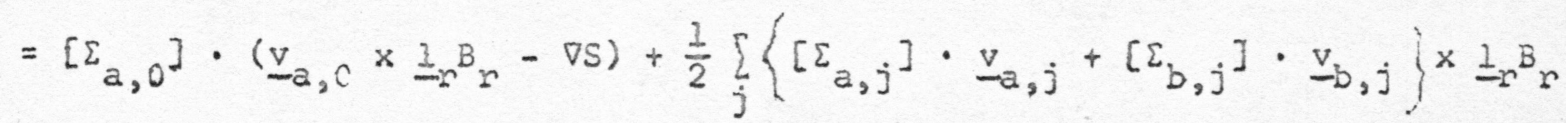
where we have used the fact that $\nabla S$ is essentially constant through the dynamo layer.

Defining $[P]$, the inverse of $\left[\Sigma_{a, 0}\right]$,

$$
\left[\Sigma_{a, 0}\right] \cdot[P] \equiv\left[\Sigma_{a, 0}\right] \cdot\left[\Sigma_{a, 0}\right]^{-1}
$$

$$
\begin{aligned}
& \equiv[1] \\
& \equiv\left(\begin{array}{ll}
1 & 0 \\
0 & 1
\end{array}\right)
\end{aligned}
$$

we may rewrite $(5,2)$ as

$I=\left[\Sigma_{a, 0}\right] \cdot\left\{v_{a, 0}+\frac{1}{2} \sum_{j}[P] \cdot\left[\Sigma_{a, j}\right] \cdot \underline{v}_{a, j}+\frac{1}{2} \sum_{j}[P] \cdot\left[\Sigma_{b, j}\right] \cdot \underline{v}_{b, j}\right\} \times \underline{u}_{r} B_{r}$

$$
-\left[\Sigma_{a, 0}\right] \cdot \nabla S
$$


An effective dynamo layer wind may now be defined as

$$
\begin{aligned}
\underline{v}_{e f f} & =\underline{v}_{a, 0}+\frac{1}{2} \sum_{j}[P] \cdot\left[\Sigma_{a, j}\right] \cdot \underline{v}_{a, j}+\frac{1}{2} \sum_{j}[P] \cdot\left[\Sigma_{b, j}\right] \cdot \underline{v}_{b, j} \\
& =\frac{1}{2} \sum_{j}\left(\left[\tau_{a, j}\right] \cdot v_{-, j}+\left[\tau_{b, j}\right] \cdot \underline{v}_{b, j}\right)
\end{aligned}
$$

where $\left[T_{a, j}\right]=[P] \cdot\left[\Sigma_{a, j}\right]$ and $\left[T_{a, j}\right] \equiv 2[1]$ and $\left[T_{b}, 0\right] \equiv 0$. This permits the dynamo equation to be written

$$
I=[\Sigma a, 0] \cdot\left(\underline{v}_{e f f} \times \underline{I}_{n} B_{r}-\nabla S\right)
$$

Now, as discussed in Chapter 2, section 5, we may separate the effective wind into a current producing, and a non-current producing component,

$$
\underline{y}_{\text {eff }}=-\frac{1}{B_{n}} \nabla \times S_{1} \frac{1}{n}-\frac{1}{B_{r}} \nabla \psi \text {. }
$$

and finaliy write the dynamo equation

$$
-\underline{I} \times \nabla \Gamma=\left[\Sigma_{a, 0}\right] \cdot\left(\underline{I}_{-\infty} \times \nabla \psi-\nabla S_{2}\right)
$$

where $\nabla S_{2}$ is the electrostatic field associated with the current producing wind system. Being a two element vector equation with two unknowns, $I$ and $S_{2},(5.8)$ can be solved. A differential equation in $\Gamma$ alone can be obtained by applying the tensor [P] to both sides of the equation and then taking the curl to obtain

$$
-\nabla \times\left\{[P] \cdot \underline{3}_{-\infty} \times \nabla \Gamma\right\}=\nabla \times\left(\underline{3}_{-2} \times \nabla \psi\right)
$$


This is equivalent to the equation solved by earlier workers (cf. Chapman and Bartels). An equation for $\mathrm{S}_{2}$ can be obtained simply by taking the divergence of both sides of $(5.8)$. Thus,

$$
\nabla \cdot\left\{\left[\Sigma_{a, 0}\right] \cdot\left(I_{-\infty} \times \nabla \psi-\nabla s_{2}\right)\right\}=0 .
$$

A third, not independent, differential equation relating the curnent function $\Gamma$ and the electrostatic potential $S_{2}$ may be obtained by multiplying both sides of $(5.8)$ by $[P]$ and then taking the divergence of all terms. This leads to

$$
\nabla \cdot\left\{\begin{array}{llll}
{[P]} & \underline{2}_{r} & \times & \nabla r
\end{array}\right\}=\nabla^{2} s_{2} .
$$

Until a model for the wind at all heights in the dynamo layer is available, equation (5.11) is the only one of the three equations having practical importance, and even its importance is rather diminished by the fact that $\nabla S_{2}$ is not the entire electrostatic field generated by dynamo action.

One approach to solving these equations for one or another of the unknowns is to expand the known functions in orthogonal functions and consider the unknown to be similarly expanded. After performing the necessary operations the coefficient of like functions can be equated to evaluate the coefficients in the expansion of the unknown. This procedure is followed here to obtain a solution of $(5.11)$ for $\mathrm{s}_{2}$, in terms of a known $\Gamma$.

Equation (5.11) can be written

$$
\begin{aligned}
\frac{1}{r \sin \theta} \frac{\partial}{\partial \lambda}\left(P_{\lambda \lambda}\right. & \left.\frac{2}{r} \frac{\partial r}{\partial \theta}-P_{\lambda \theta} \frac{1}{r \sin \theta} \frac{\partial r}{\partial \lambda}\right)+\frac{1}{r \sin \theta} \frac{\partial}{\partial \theta}\left(P_{\theta \lambda} \frac{\sin \theta}{r} \frac{\partial \Gamma}{\partial \theta}-P_{\theta \theta} \frac{1}{r} \frac{\partial \Gamma}{\partial \lambda}\right) \\
& =\frac{1}{r^{2} \sin ^{2} \theta} \frac{\partial^{2} S_{2}}{\partial \lambda^{2}}+\frac{1}{r^{2} \sin \theta} \frac{\partial}{\partial \theta}\left(\sin \theta \frac{\partial S_{2}}{\partial \theta}\right)
\end{aligned}
$$


Defining operators $B$ and $D$ such that

$$
\begin{aligned}
& B f(\theta, \lambda) \equiv \sin \theta \frac{\partial}{\partial \theta} f(\theta, \lambda) \\
& D f(\theta, \lambda) \equiv \frac{\partial}{\partial \lambda} f(\theta, \lambda),
\end{aligned}
$$

and multiplying both sides of $(5.12)$ by $r^{2} \sin ^{2} \theta$, we may rewrite (5.12)

$$
D\left(P_{\lambda \lambda} B \Gamma-P_{\lambda \theta} D \Gamma\right)+B\left(P_{\theta \lambda} B \Gamma-P_{\theta \theta} D \Gamma\right)=\left(D^{2}+B^{2}\right) S_{2}
$$

The form of the operators $B$ and $D$ will be determined by the basis chosen for the linear vector space in which $\Gamma$ and $S_{2}$ are defined. Hasegawa and Ota (1948) have already derived a representation for the magnetic potential $W$ of the $\mathrm{Sq}$ magnetic variation using as a basis the surface hamonic functions $\cos m \lambda P_{n}^{m}(\cos \theta)$ and $\sin m \lambda P_{n}^{m}(\cos \theta)$, and from this a representation of the current function $r$ may be obtained. If

$$
W(\theta, \lambda)=\sum_{n=0}^{\infty} \sum_{m=0}^{n}\left(w_{a, n}^{m} \cos m \lambda+w_{b, n}^{m} \sin m \lambda\right) P_{n}^{m}(\cos \theta)
$$

the curnent function $\Gamma$ is given (Chapman and Bartels, II, p. 631, 1940) by

$\Gamma(\theta, \lambda)=-\frac{10}{4 \pi} \sum_{n=0}^{\infty} \frac{2 n+1}{n+1}\left(\frac{n}{a}\right)^{n} \sum_{m=0}^{n}\left(w_{a, n}^{m} \cos m \lambda+w_{b, n}^{m} \sin m \lambda\right) P_{n}^{m}(\cos \theta)$

where $W(\theta, \lambda)$ is the magnetic potential at the surface of a sphere of radius a which is less than $r_{0^{2}}$ the radius of the spherical surface on which the sheet currents are distributed. Thus, surface harmonics would seem to be a likely choice for the basis of the representation. However, it is desired to represent the resistivity tensors [P] in the same Iinear vector space, and as indicated by $(5.14)$, products of the form $P_{\lambda \lambda}(\theta, \lambda)$ $B \Gamma(\theta, \lambda)$ must be performed and then operated upon. Such a multiplication 
of functions in the spherical hamonic representation would require a convolution involving, among other things, the representation in terms of associated Legendre polynomials of products such as $P_{n}^{m}(\cos \theta) P_{n}^{m^{\prime}}(\cos \theta)$. Identities fon such products have been worked out by Adans (1900), but they are exceedingly tedious. It is more convenient to perform the multiplication with a different basis for the representation, as is discussed in the following section.

\subsection{Representations, Transformations and Operators}

Let us suppose a real function $F(\theta, \lambda)$ is expanded in spherical harmonics in the form

$$
F(\theta, \lambda)=\sum_{n=0}^{\infty} \sum_{m=-n}^{\infty} f_{n}^{m} e^{i m \lambda} P_{n}^{|m|}(\cos \theta) .
$$

where $f_{n}^{-m}$ will be the complex conjugate of $f_{n}^{m}$. The expansion coefficients $f_{n}^{m}$ may be amanged in a two dimensional array with the index $m$ determining the column and $n$, the now. The conjugate relationship between $f_{n}^{-m}$ and $f_{n}^{m}$ permits the powtion of the array with negatively indexed columns to be suppressed, but its presence must be understood when performing certain mathematical operations. We will tem such an amay the $(n, m)$ representation of $F$ and symbolize it by $F(n, m)$. Another representation of $F(\theta, \lambda)$ can be made using the functions $\sin |m| \theta \cos ^{j} \theta$ in place of the associated Legendre functions $\mathrm{P}_{\mathrm{n}}^{\mathrm{m}}(\cos \theta)$.

The array $F(j, m)$ will have elements $f_{j}^{m}$ such that

$$
F(\theta, \lambda)=\sum_{j=0}^{\infty} \sum_{m=-\infty}^{\infty} f_{j}^{m} e^{i m \lambda} \sin |m| \theta \cos ^{j} \theta .
$$

where again $f_{j}^{m}$ and $\mathrm{f}_{j}^{-m}$ are complex conjugates and the Index $m$ will continue to specify the column in which the element is located. 
Since (cf. Chapman and Bartels (1940) equations $17.10,17.19$ and $17.20)$

$$
\begin{aligned}
P_{n}^{m}(\cos \theta)=\sin |m| & \left\{\frac{\left(2-\delta_{m 0}\right)(n-|m|) !}{(n+|m|) !}\right]^{1 / 2} \\
& \cdots\left\{\begin{array}{cc}
{\left[\left[\frac{n-|m|}{2}\right]\right]} & \frac{(2 n-2 n) !(-1)^{r}(\cos \theta)^{n-|m|-2 n}}{2^{n} n !(n-|m|-2 n) !(n-n) !}
\end{array}\right\}
\end{aligned}
$$

where $\delta_{m o}$ is the Kronecker delta and the double brackets [ []$]$ indicate greatest integer, the tnansformation from the $(n, m)$ representation to the $(j, m)$ can be effected by a transformation array $[\mathrm{g} / \mathrm{m} /]$

$$
F(j, m)=[?|m| j \cdot F(n, m)
$$

with the $|\mathrm{m}|$ superscript of $T$ indicating that a different transformation matrix $[\mathrm{T} / \mathrm{m} /]$ is necessary to transform each column of the representation array. The transformation matrix $[\mathrm{T} / \mathrm{m} \mid \mathrm{]}$ will have elements $\mathrm{T}|\mathrm{m}|$ given by

$$
\begin{aligned}
T_{j n}^{|m|}=\left\{\frac{\left(2-\delta_{m 0}\right)(n-|m|) !}{(n+|m|) !}\right\}^{1 / 2} \frac{(n+|m|+j) !(-1)(n-|m|-j) / 2}{\left.2^{n}[n-|m|-j) / 2\right] ! j ![(n+|m|+j) / 2] !} \\
\text { for } n+|m|+j \text { even, } j \leq n-|m| \\
=0 \text { otherwise }
\end{aligned}
$$

In the following we assume that an adequate representation of $F(\theta, \lambda)$ is obtained with a finite number of tems $k$. Suppose that $G(\theta, \lambda)$ is Iikewise reprosented by $G(j, m)$ and we wish to determine the $(j, m)$ 
zepresentation of the function $H(\theta, \lambda)$ defined by the relationship

$$
H(\theta, \lambda) \equiv E(\theta, \lambda) G(\theta, \lambda) \text {. }
$$

In terms of the basic functions of the $(j, m)$ representation this may be written

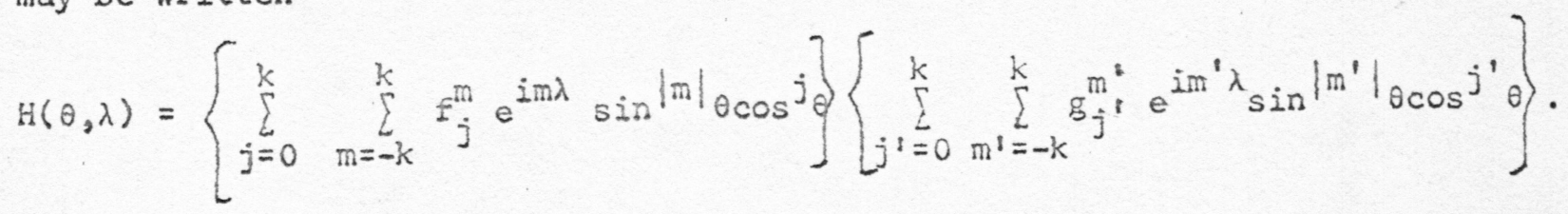

Consider the four terms arising from the multiplication of the two terms containing the coefficients $f_{j}^{m}$ and $f_{j}^{-m}$ by the two terms with coefficients $g_{j}^{\prime}$ and $g_{j}^{-m^{\prime}}$. These will be.

$$
\begin{aligned}
& f_{j}^{m} g_{j^{\prime}} e^{i\left(m+m^{\prime}\right) \lambda}(\sin \theta)^{|m|+\left|m^{\prime}\right|}(\cos \theta)^{j+j^{\prime}} \\
& f_{j}^{m} g_{j^{\prime}}^{-m^{\prime}} e^{i\left(m-m^{\prime}\right) \lambda}(\sin \theta)^{|m|+\left|m^{\prime}\right|}(\cos \theta)^{j+j^{\prime}} \\
& f_{j}^{-m} g_{j^{\prime}}^{m^{\prime}} e^{-i\left(m^{\prime}-m^{\prime}\right) \lambda}(\sin \theta)^{|m|+\left|m^{2}\right|}(\cos \theta)^{j+j^{\prime}} \\
& f_{j}^{-m} g_{j^{\prime}}^{-m^{\prime}} e^{-i\left(m+m^{2}\right) \lambda}(\sin \theta)|m|+\left|m^{\prime}\right|(\cos \theta)^{j+j^{\prime}}
\end{aligned}
$$

Let us suppose that $m>m^{p}>0$. Then terms (a) and (b) would give contributions to tems of positive $m$ index in $H(j, m)$, the representation of the product $H(\theta, \lambda)$. Terms (c) and (d) have negative index $m$ and are the complex conjugates of $(b)$ and (a) respectively. Consequently they may be suppressed as discussed earlier. If, on the other hand, $m^{3}>m>0$ terms (a) and (c) would contribute to the terms of positive index $m$ while terms (d) and (b), their respective complex conjugates, would be suppressed. In the case of $m=m^{\prime}>0$ tems $(b)$ and $(c)$ would both contribute to the $m=0$ terms of $H(j, m),(a)$ would contribute to the terms of index $2 m$, and 
(c), its complex conjugate, would be suppressed. In the case where $m>m^{i}=0$ there would be only two terms, one with $e^{i m \lambda}$ as a factor, and the other with $e^{-i m \lambda}$. The former contributes to the terms of index $\mathrm{m}$ of the product and the latter may be suppressed. Finally, if $m=m^{\prime}=0$ only one term occurs and is a contribution to the $m=0$ term of the product.

Terms (b) and (c), those involving the multiplication of positive and negative index $m$ (we may assume $m \geq m^{i} \geq 0$ without loss of generality), are not in the correct form to be considered terms of the $(j, m)$ representation because they contain powers of $\sin \theta$ that are not the same as the power of $e^{i \lambda}$. If $m>m^{\circ}$ we may write

$$
(\sin \theta)^{m+m^{\prime}}=(\sin \theta)^{m-m^{\prime}}\left(1-\cos ^{2} \theta\right)^{m^{\prime}}
$$

allowing $(6.8 b)$ to be rewritten

$$
m_{j}^{m} g_{j^{\prime}}^{-m^{\prime}} e^{i\left(m-m^{\prime}\right) \lambda}(\sin \theta)^{m-m^{2}} \sum_{r=0}^{m^{\prime}}(-1)^{r} \frac{m^{2} !}{\left(m^{\prime}-r\right) ! r !}(\cos \theta)^{j+j^{\prime}+2 r}
$$

which is of the desired form.

From the above discussion the kind of matrix multiplication necessary to obtain the representation $H(j, m)$ from the matrices representing $F(j, m)$ and $G(j, m)$ may be deduced. This matrix product will be indicated by

$$
H(j, m)=F(j, m) * G(j, m)
$$

and will involve a product combination of each column of the matrix $F(j, m)$ with each column of $G(j, m)$, and the addition of the result into the proper column of $H(j, m)$. We will here call each such combination a columnar product. 
To obtain the columnar product of two column factors, one of the columns is transposed into a row and is placed to the right of the other. Then a standard matrix multiplication between the column and row is performed

$$
\left(\begin{array}{l}
f_{0} \\
f_{1} \\
f_{2}
\end{array}\right)\left(\begin{array}{lll}
\left(g_{0}, g_{1}, g_{2}\right) \\
f_{1} g_{0} & f_{1} g_{1} & f_{1} g_{2} \\
f_{2} g_{0} & f_{2} g_{1} & f_{2} g_{2}
\end{array}\right)
$$

and the columnar product formed by summing on the anti-diagonals, adding together all terms whose component factors have $j$ indices summing to the same value. Thus, from $(6.11)$ the resulting columnar product is

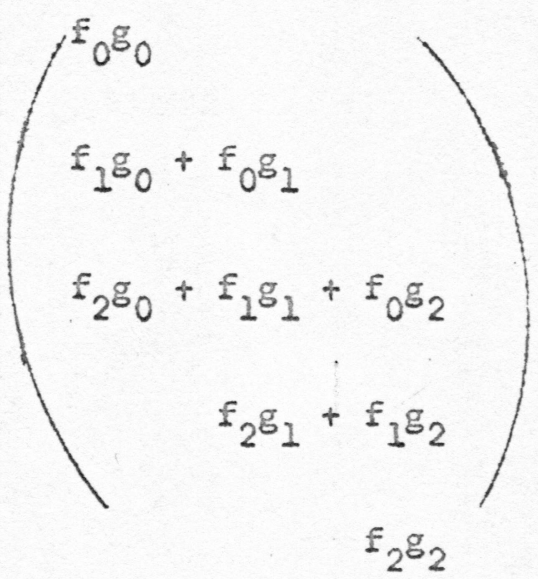

Note that it has $(2 j+1)$ rows where the columnar factors each had only $j$. A columar product of this kind is obtained from each paired combination of columns of $F(j, m)$ and $G\left(j^{n}, m^{p}\right)$ and the columnar product is added to the product matrix $H(j, m)$ in the $m+m^{\prime}$ column.

It is also necessary to take account of the suppressed columns with negative index. This is best accomplished at the same time as the columnar products involving the columnar factors with comesponding in index are taken. 
Let us suppose the columnar product of column $m$ of $F(j, m)$ and column $m^{\prime}$ of $G(j, m)$ has just been added into the $m+m^{\prime}$ column of $H(j, m)$, and further suppose $m \geq m^{\prime}$. The elements of column $m^{\prime}$ of $g(j, m)$ are replaced by their complex conjugates and the columnar product of this conjugated column and the column $\mathrm{m}$ of $\mathrm{F}(j, \mathrm{~m})$ is formed. Next a columnar multiplication of the columnar product just obtained, by the $m^{\prime}$ column of the matrix $M(j, m)$ is performed and the result added to the $m-m^{\prime}$ column of $H(j, m)$.

The matrix $M(j, m)$ is determined from equation (6.8b') and has elements $\mathbf{m}_{j}^{\mathrm{m}}$ given by

$$
\begin{aligned}
& m_{j}^{m}=(-1)^{j / 2} \frac{m !}{(m-j / 2) !(j / 2) !} \text { for } j \text { even, } j \leq 2 m \\
& m_{j}^{m}=0 \text { otherwise }
\end{aligned}
$$

In the case where $m^{i} \geq m$ the roles of the two columnar factors are reversed, so the $m$ column of $F(j, m)$ has its element replaced by their complex conjugates. Also, instead of the $m^{P}$ column of $M$, the $m$ colum of $M$ is used. The result is then added into the $\left(m^{p}-m\right)$ column of $H(j, m)$. It is important to note that both procedures must be followed if $m=m^{p}$ since two contributions to $h_{j}^{\circ}$ are required (see discussion following equation 6.8).

Having developed the transformation from the surface harmonic $(n, m)$ representation to the $(j, m)$ representation, and the proper method for taking the convolution product of functions in the $(j, m)$ representation, we must next find the matrix representation of the operators $B$ and $D$. The operation $D$, which takes the derivative of a function $F(\theta, \lambda)$ with respect to $\lambda$ may be carried out by simply multiplying each element of $F(j, m)$ by the $m$ index of the element and by $i \equiv \sqrt{-1}$. This may also be 
regarded as the result of performing a standard matrix multiplication of each column of $F(j, m)$ by a matrix $D^{m}$ corresponding to that column, where

$$
D^{m}=i m[1]
$$

and $[1]$ is the identity matrix.

The $B$ operator takes the derivative of $F(\theta, \lambda)$ with respect to $\theta$ and then multiplies by $\sin \theta$. Thus

$$
\begin{aligned}
B e^{i m \lambda} \sin |m|_{\theta} & \cos ^{j} \theta=\sin \theta \frac{\partial}{\partial \theta}\left(e^{i m \lambda} \sin ^{|m|} \theta \cos ^{j} \theta\right) \\
& =e^{i m \lambda} \sin ^{|m|}\left\{(|m|+j) \cos ^{j+1} \theta-j \cos ^{j-1} \theta\right\}
\end{aligned}
$$

and the necessary operation is accomplished by the standard matrix multiplication of each column by a corresponding matrix $B^{|m|}$ where the $j$ th column of $B^{|m|}$ has elements $(|m|+j)$ and $-j$ in rows $j+1$ and $j-1$ respectiveiy, while all other elements of the $j$ th column are zeros. There will be a different matrix $B|m|$ for each column of the $(j, m)$ representation, and $B|m|$ as well as $D^{m}$, is diagonal with respect to the $m$ index (that is, an element in the mth column of $F(j, m)$ contributes only to the mth column of $\operatorname{Dr}(j, m)$ or $\operatorname{Br}(j, m)$.

This gives us the complement of operations and operators needed to solve equation (5.14). A solution for $S_{2}$ can be effected with the inversion of the operator $\left(B^{2}+D^{2}\right)$.

3.7 Undetected Zonal Currents and the Effect of Their Omission on Solutions for the Electrostatic Field

Although in principle it is possible, by spherical harmonic analysis, to separate the earth's field into parts of external and internal origin, the portion of the earth's unvarying magnetic field attributable to external 
sources is not well known because of the limited accuracy of such analyses. One possibly lange extemal source of such fields is a remnant ring current produced by the differential drift of charged particles always trapped in the magnetosphere. However, here the possibility of a contribution to the earth's unvarying field by currents in the dynamo region will be considered. In the case of symmetric dynamo action, such currents would have to be zonal, as symmetric meridional curnent systems independent of longitude would be divergent and could not exist in the steady state. Such zonal current systems, giving rise to magnetic perturbations of some tens of gammas, might exist in the jonosphere within the uncertainty of the deternination of the component of field of extennal origin.

The mechanism for currents with such a configuration certainly exists as well. Consider the current driven directly by dynamo action.

$$
\underline{J}_{D}=\left[\Sigma_{0}\right] \cdot \underline{v e f f}^{x} \underline{n}_{n}{ }^{B}
$$

If $\left[\varepsilon_{0}\right]$ and $\mathrm{v}$-eff are expanded in spherical harmonics and the indicated multiplications carried out, the multiplication of terms of the same order (index $\mathrm{m}$ ) will give rise to terms of order zero in the expansion for the current. Consequently, besides the contribution from menidional circulation in the dynamo region combined with the zero onder components of conductivity, one can anticipate a sizable contribution to the zero order component of current from the combined effect of the large diumal component of conductivity and any diumal component of the current producing portion of the wind velocity field. Similarly, matched higher order components of conductivity and wind can be expected to contribute to the zonal (and meridional) currents as well. Any zero order meridional component of (symmetric) cursent would have to be balanced by an equal and 
opposite zero onder meridional component driven by an electrostatic field, and because of the lange diumal component of conductivity, such an electric field can itself be expected to have a sizable diurnal component. That this is indeed the case may be seen by referring to equation (5.14)

$$
D\left(P_{\lambda \dot{\lambda}} B \Gamma-P_{\lambda} \ddot{\theta} D \Gamma\right)+B\left(P_{\theta} * B \Gamma-P_{\theta} \dot{\theta} D \Gamma\right)=\left(D^{2}+B^{2}\right) S_{2}
$$

If the current function $\Gamma$ has a zero order component in its surface harmonic expansion and [P] has a large diumal component, $S_{2}$ will clearly have a corresponding diurnal component, since the operators $B$ and $D$ do not change the order of a term of the surface hamonic, while multiplication by the large diurnal $(m=1)$ term of $[P]$ produces diurnal components from the zero onder term it multiplies.

From the foregoing discussion it may be concluded that in computing the electrostatic field from the observed Sq magnetic variation, there is likely to be an important contribution to the diurnal terms of the electrostatic potential (and to other terms, as well) which is missed because the magnetic fields arising from zonal currents cannot be easily detected. 


\subsection{Antisymmetric Dynamo Action}

As was the case for symmetric dynamo action, it is possible to carry out the discussion of antisymmetric dynamo action entirely in terms of the processes in one hemisphere, provided suitable terminations to the field lines are introduced at the equatorial plane. As discussed in Chapter 2, antisymmetric dynamo action will give rise to currents along the magnetic field, but to no electrostatic field transverse to the field lines in the equatorial plane. Such conditions are met by a short-circuit termination, or in other words, by considering the equatorial plane to be filled by a perfectly conducting sheet.

As a consequence of this, the electrostatic fields arising from the divergence of the antisymetric dynamo driven current need not grow sufficiently large to drive a current system in the dynamo layer that cancels the divergence of the dynamo driven current, as was the case with symmetric dynamo action. Now the possibility exists for there to be currents outward along the field lines from regions where the dynamo driven currents converge and inward where they diverge. Here it will first be demonstrated that for wind systems of global scale these currents along the field lines will be sufficient to provide a divergence-free total current system while driven by electrostatic fields of insufficient magnitude to produce appreciable current transverse to the field lines. To demonstrate this, the resistance of a flux tube from the ionosphere to the equatorial plane will be compared with the resistance in the ionosphere between a ring bounding the flux tube 
and the zero potential sunface within the ionosphere, here taken to be a concentric ring with radius twice that of the flux tube (see Fig. 3 ).

It is first necessary to compute the resistance of a unit tube of magnetic flux extending from the dynamo layer to the equatorial plane. The tubes of flux will increase in cross section greatly with increased geocentric distance, so a unit tube of flux will be defined as one having unit cross section at the height of the dynamo layer. The resistance of an incremental length along the tube of flux will be

$$
d R=\left\{\sigma_{0}(r) A(r, \theta)\right\}^{-1} d s
$$

where $R$ is the resistance, $\sigma_{0}(r)$ the conductivity along the field line assumed uniform over the globe, $A(r, \theta)$ the cross section of the tube of Flux, and $s$ is distance measured along the lines of force. To obtain the total resistance from the dynamo layer to the equatorial plane it is necessary to integrate along the field lines.

$$
R=\int_{r_{0}}^{\infty}\left\{\sigma_{0}(r) A(r, \theta)\right\}^{-1}(d s / d r) d r
$$

where the variable of integration is $r$, the geocentric distance, and where $r_{0}$ is at the dynamo layer and $r_{e}$, at the point where a particular field line intersects the equatorial plane. The cross section of a tube of flux is inversely proportional to the magnitude of the magnetic induction $B$, so we may write

$$
A(r, \theta)=A\left(r_{0}, \theta_{0}\right) B\left(r_{0}, \theta_{0}\right) / B(r, \theta) .
$$




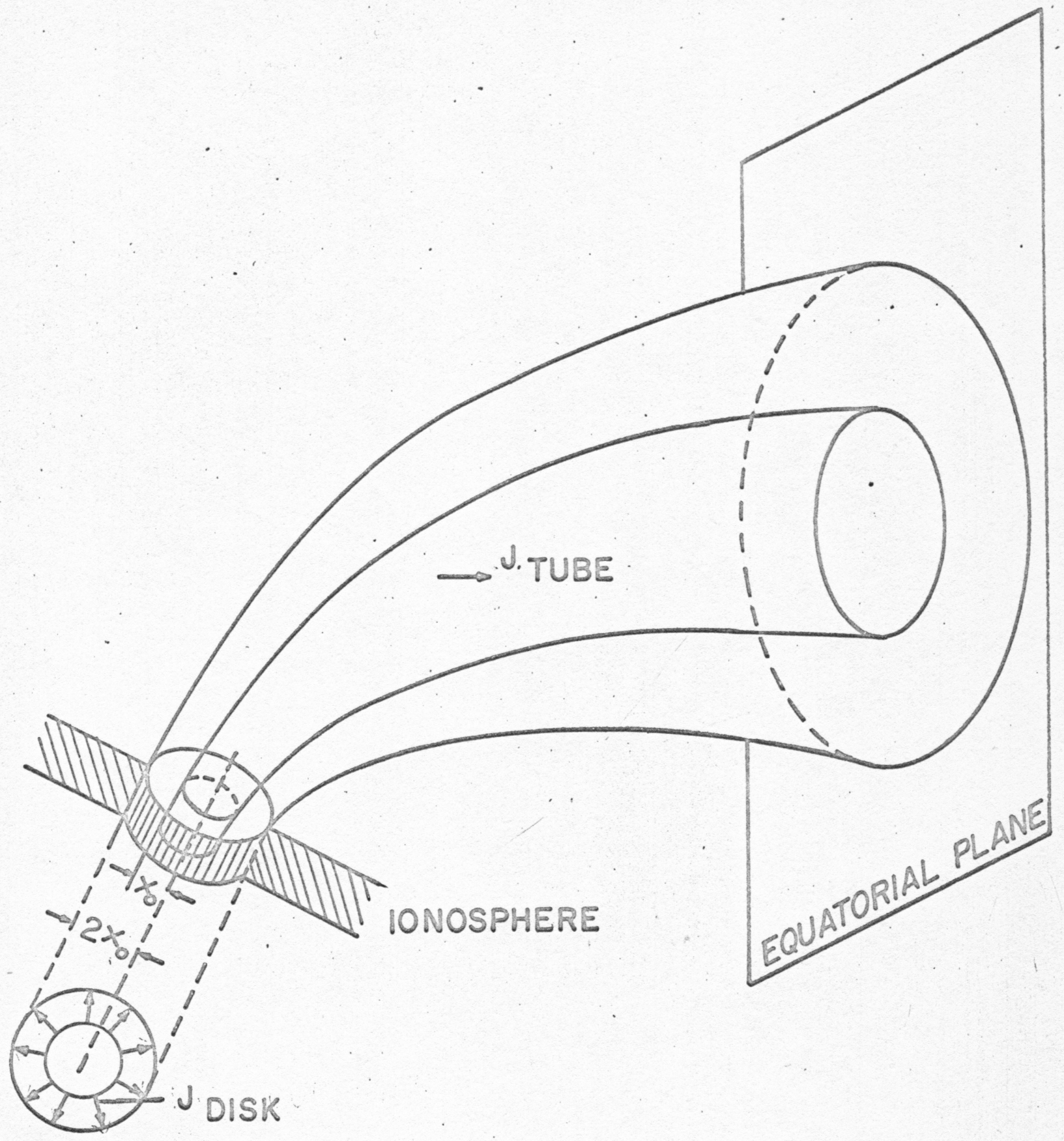

Fig. 3 Geometry for the comparison of the relative magnitudes of currents along the field line to the equatorial

plane and currents transverse to the magnetic field in the ionosphere under antisymmetric dynamo action. The resistance of the inner tube from the ionosphere to the equatorial plane (Rtube) is compared to that of the ionosphere between the inner and outer tube. $\left(R_{\text {disc }}\right)$. 
Making use of the assumption that the magnetic field is dipolar and that the tube of flux has unit cross section at $r_{0}$, we obtain

$$
A(r, \theta)=\left(r / r_{0}\right)^{3}\left(4 r_{0}-3 r_{0} \sin ^{2} \theta_{0}\right)^{\frac{1}{2}}\left(4 r_{0}-3 r \sin ^{2} \theta_{0}\right)^{-\frac{1}{2}}
$$

Similarly, the dipolar property of the field can be used to obtain

$$
d s / d r=\left(4 r_{0}-3 r \sin ^{2} r_{0}\right)^{\frac{3}{2}}\left(4 r_{0}-4 r \sin ^{2} r_{0}\right)^{-\frac{1}{2}}
$$

Substituting these into the integral one obtains

$$
R\left(r_{0}, \theta_{0}\right)=\int_{r_{0}}^{\left(r_{0} / \sin ^{2} \theta_{0}\right)} \frac{1}{\sigma_{0}(r)} \frac{r_{0}^{3}}{r} \frac{\left(4 r_{0}-3 r \sin ^{2} \theta_{0}\right)}{\sqrt{\left(4 r_{0}-3 r_{0} \sin ^{2} \theta_{0}\right)\left(4 r_{0}-4 r \sin ^{2} \theta_{0}\right)}} d r
$$

In Fig. 4 is shown $R$ plotted as a function of $\theta$ for three model atmospheres, appropriate for noon, twilight and midnight, and for several heights in the dynamo region. The conductivity $\sigma_{0}$ used here was computed from model atmospheres of Prince, Bostick and Smith (1964).

Next the resistance within the dynamo layer may be estimated. Consider the resistance between the bounding ring of the flux tube of radius $x_{0}$, and the outer ring (Fig. 3 ) which is taken to be at zero potential, and to have a radius $2 x_{0}$. The resistance of an incremental ring lying between the two is

$$
d R_{d i s c}=\left(2 \pi h \sigma_{1} x\right)^{-1} d x
$$


Fig. 4 The resistance of a magnetic flux tube having a square meter cross section at the ionosphere. The resistance between the 100, 110, 120 and $130 \mathrm{~km}$. levels and the equatorial plane is plotted as a function of colatitude. Fig. 4a is for a model appropriate for noon time conditions, $4 \mathrm{~b}$ for twilight conditions, and $4 \mathrm{c}$ for midnight conditions. 


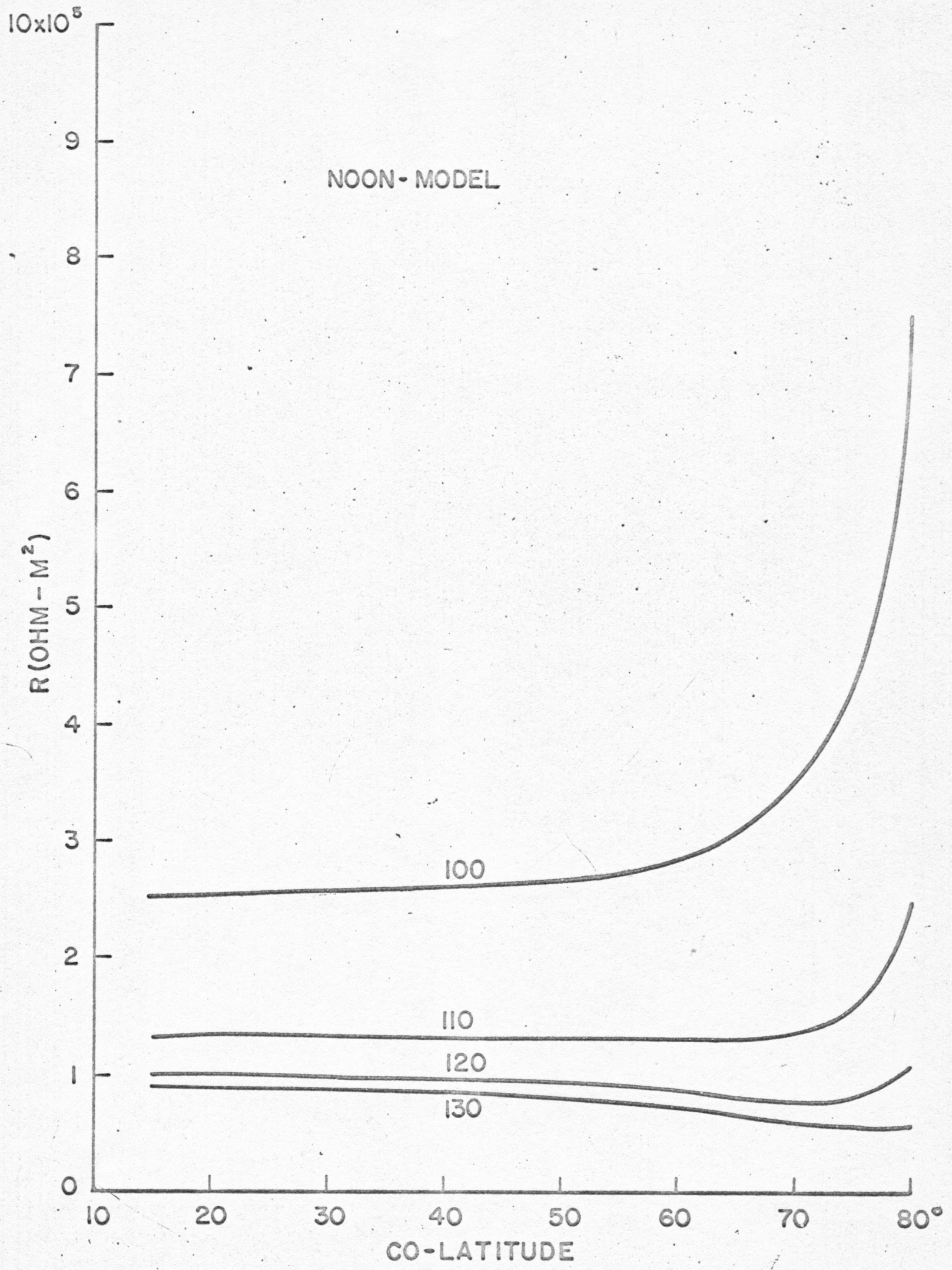




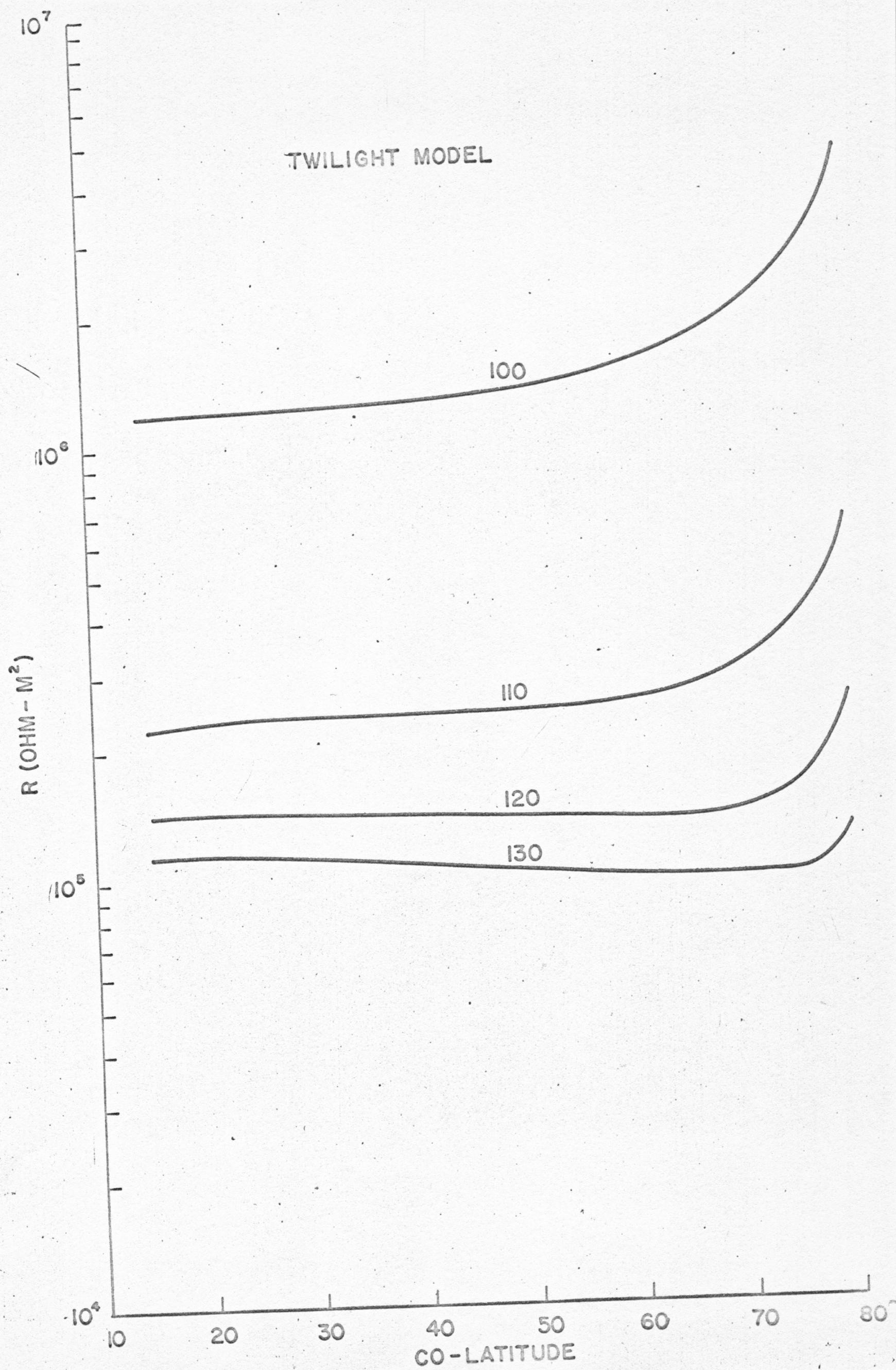




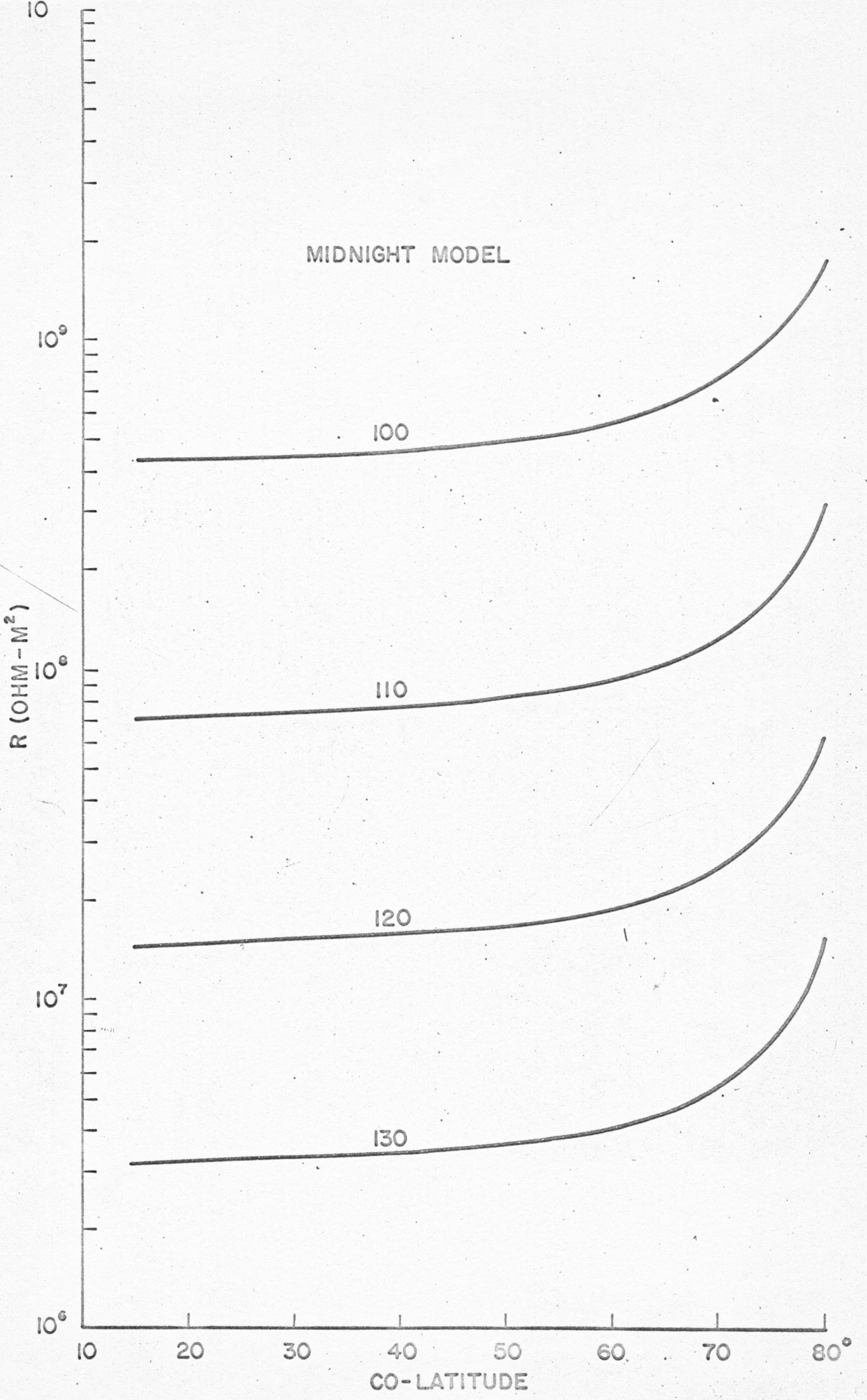


where $h$ is the thickness of the dynamo layer and $\sigma_{1}$ is the mean Pedersen conductivity. Integrating from $x_{0}$ to $2 x_{0}$ one obtains

$$
R_{\text {disc }}=\left(2 \pi h \sigma_{1}\right)^{-1} \text { in } 2
$$

On the other hand, the total of resistance between the equatorial plane and the circular region of the dynamo layer bounded by the inner ring is

$$
R_{\text {tube }}=\frac{R_{2}}{\pi x_{0}^{2}}
$$

where $R$ is the total resistance along a unit flux tube found from (1.6). At middle latitudes the total resistance (from $100 \mathrm{~km}$ height to the equatorial plane) of a unit flux tube at mid latitudes nean the noon meridian is about $3 \times 10^{5}$ ohms, while the Pedersen conductivity is of order $3 \times 10^{-4} \mathrm{ohms} / \mathrm{meter}$ over a height range $\mathrm{h}$ of scale $5 \times 10^{4}$ meters. Equating $R_{\text {disc }}$ of (1.8) to $R_{\text {tube }}$ of (1.9) and solving for $x_{0}$ it is found that for structure in the dynamo layer having horizontal scale greater than a few kilometers the conduction along the field lines to the equatorial plane is more important than conduction transverse to the field in the dynamo layer. The effectiveness of the conduction along the field increases with the square of the horizontal scale size, while horizontal scale has no influence on $R_{\text {disc }}$. Thus, for antisymmetric systems having horizontal scales of onder $10^{3} \mathrm{~km}$ there will be negligible current driven transvense to the magnetic fields by the electrostatic field. 
4.2 Currents Driven by Antisymmetric Dynamo Action

In the preceding section it was found that in the case of antisymmetric dynamo action the electrostatic field gives rise to little current transverse to the magnetic field compared to that driven along the field lines to the equatorial plane. For all practical purposes, the electrostatic field driven current $J_{-E}$ may here be taken to be entirely in the direction of the magnetic field, while on the other hand, the dynamo driven current, $J_{-D}$, is by its very nature orthogonal to the magnetic field.

Substituting $J_{-D}$ for $J$ in equation 4.22 of Chapter 3 gives an expression for the total divengence of the dynamo driven current in the truncated conical volume extending through the dynamo layen over which the integration is carried.

$$
\int_{r_{0}}^{r}\left(r / r_{0}\right)^{2} \nabla \cdot J_{D} d r=\nabla \cdot I D h
$$

Any divergence of $J_{D}$ in the layen must be balanced by equal and opposite divergence of $\mathrm{J}_{\mathrm{E}}$. Although to be precise it would be necessary to consider the total divergence of the dynamo driven current in any such truncated conical volume to be balanced by divergence of $\mathrm{J}_{\mathrm{E}}$ distributed in some fashion among all the tubes of flux passing through the volume, we will here consider such divergence to be matched entirely within the flux tube intersecting the level $r_{0}$ in the same meter square area that forms the lower base of the truncated cone. The error in position introduced by doing this will be small compared to the horizontal scale of the current and wind systems of interest to us, except quite near the equator where the magnetic field has little dip. With this approximation we may write 


$$
\underline{J}_{E} \sin x=\nabla \cdot \underline{I}_{D h}
$$

or by writing $\sin x$ in terms of $\theta$

$$
J_{E}=\frac{\left(I+3 \cos ^{2} \theta\right)^{1 / 2}}{2 \cos \theta} \nabla \cdot I_{D h}
$$

The direction of $J_{E}$ will be downward along the field lines toward the base of those truncated conical volumes in which there is positive divergence. The current density passing through the equatorial plane on a field line that intersects $r_{0}$ at $\theta$ will be denoted $J_{e q}(\theta, \lambda)$ and is given by

$$
\begin{aligned}
J_{e q}(\theta, \lambda) & =\left\{B_{e}(\theta) / B_{0}(\theta)\right\} \quad J_{E}(\theta, \lambda) \\
& =\frac{\sin ^{6} \theta}{2 \cos \theta} \nabla \cdot I_{D h}
\end{aligned}
$$

Here $\left\{B_{e}(\theta) / B_{0}(\theta)\right\}$ is the ratio of the magnetic induction (and thus the inverse ratio of the area of the flux tube) at the point where a field line crosses the equatorial plane to that at the point where it intersects the level $r_{0}$

The current along the field lines may next be related to the two kinds of wind systems that have previously been referred to as the current producing wind system and the non-current producing wind system. As already noted, in the antisymmetric case both of these wind systems produce current and contribute to the $\mathrm{Sq}$ magnetic variation.

In terms of the components of the horizontal wind, the horizontal components of the dynamo driven current may be written 


$$
\begin{aligned}
\underline{J}_{D h} & =\underline{I}_{\theta}\left(\sigma_{1} v_{\lambda} B_{r}+\sigma_{2} B_{r}^{2} v_{\theta} / B+\underline{I}_{\lambda}\left(\sigma_{2} v_{\lambda} B-\sigma_{1} v_{\theta} B_{r}\right)\right. \\
& =[g] \cdot \underline{v} \times \underline{I_{r}} B_{r}
\end{aligned}
$$

where

$$
\begin{aligned}
& g_{\theta \theta}=g_{\lambda \lambda}=\sigma_{1} \\
& g_{\theta \lambda}=\sigma_{2} \sin x \\
& g_{\lambda \theta}=-\sigma_{2} / \sin x .
\end{aligned}
$$

Note that this conductivity differs from the tensor conductivity for symmetric dynamo action because the vertical current is not impeded. As was done in deriving the layer conductivities, the tensor [g] and wind velocity $\underline{v}$ may be expanded in terms of their radial spectra, and the integrated layer current may be written

$$
\begin{aligned}
& I_{D h}=\left[G_{a, 0}\right] \cdot v_{a, 0} \times I_{-r}{ } r_{r}+\frac{I}{2} \sum_{j}\left[G_{a, j}\right] \cdot v_{a, j} \times I_{-2} B_{r} \\
& +\frac{1}{2} \sum_{j}\left[G_{b, j}\right] \cdot \underline{v}_{b, j} \times \frac{1}{-B} B_{r} \\
& =\left[G_{a, 0}\right] \cdot \underline{v e f f} \times \frac{1}{-r} B_{r}
\end{aligned}
$$

where the effective velocity $v_{0 f f}$ for the antisymmetric case is given by the equation

$$
\begin{aligned}
v_{e f f}=v_{a, 0} & +\frac{1}{2} \sum_{j}\left[G_{a, 0}\right]^{-1} \cdot\left[G_{a, j}\right] \cdot v_{a, j} \\
& +\frac{1}{2} \sum_{j}\left[G_{a, 0}\right]^{-1} \cdot\left[G_{b, j}\right] \cdot v_{b, j}
\end{aligned}
$$

The pontion of $\underline{v}_{0 f f}$ of the form $v_{-1}=\left(1 / B_{r}\right) I_{-r} \times \nabla \xi$ will produce $a$ current 


$$
J_{e q}(\theta, \lambda)=\left\{\left(\sin ^{6} \theta\right) /(2 \cos \theta)\right\} \nabla \cdot\left[G_{a, 0}\right] \cdot \nabla \xi
$$

Similarly, the portion of $\underline{v}_{\text {eff }}$ of the form $\underline{v}_{-2}=-\left(1 / B_{n}\right) \nabla \psi$ will give rise to a layer integrated current of the form

$$
I_{D h}=\left[G, a_{a}\right] \cdot I_{n} \times \nabla \psi
$$

and to a current through the equatorial plane

$$
J_{e q}(\theta, \lambda)=\left\{\left(\sin ^{6} \theta\right) /(2 \cdot \cos \theta)\right\} \nabla \cdot\left[G_{a, 0}\right] \cdot I_{-\infty} \times \nabla \psi
$$

These equations may conveniently be treated in terms of the $(j, m)$ representation in a manner generally similan to that discussed in section 3.6 .

\subsection{Magnetic and Dynamic Effects of Antisymmetric Dynamo Action}

Antisymmetric dynamo action, while giving rise to no motion of the magnetospheric plasma, will however produce two effects having some geophysical significance. One of these is a minor effect on the dynamics of the atmosphere at the dynamo layer, represented by the Lorentz force $\mathrm{J} \times \underline{B}$ associated with the antisymmetric current systems. Chapman and Bartels, Vol. II (1940) list the coefficients of the spherical harmonic terms for the solar daily variation of the magnetic field. Among the terms attributable to antisymetric dynamo action (1isted by Chapman and Bartels as the solstitial semi-differences $\frac{1}{2}(N-S)$ ) are terms having magnitudes of orden $\frac{1}{3}$ those of the terms arising from symmetric dynamo action. Correspondingly, their Lorentz force in the dynamo layer will be about one thind that of the symmetric component, or about one thind newton/square 
meter column. Although this force seems to be smaller than others present in the dynamo layer, its interpretation is nonetheless interesting, for it may be considered to be a force transmitted from one hemisphere to the other by the hydromagnetic action of the magnetosphere.

The second effect is an antisymmetric distortion of the magnetic field, which may be thought of as a twisting of large magnetic flux tubes arising from the equal but opposite forces exerted on them where they pass through the dynamo region. This phenomena would have a small effect in changing the relative positions at which any given field line intersects the earth, causing a displacement of the geomagnetic conjugate points from the position calculated using an average model of the main field. Such displacements will be greatest for field lines emanating from the polar region, since these field lines are within the magnetospheric tail and thus have a great enough extent that the twisting can produce a somewhat more appreciable angular displacement.

Consider a ring in the dynamo region that forms the equatorward limit for the pontion of the dynamo layen intersected by the field lines that connect to the magnetospheric tail (see Fig. 5). Let us suppose that an antisymmetric meridional cument driven by dynamo action passes into (or out of) this ring and that this current has a layen integrated density of about 5 amperes per kilometer. The total current passing into for out of) such a ring, which we will here approximate as being the $75^{\circ}$ circle of latitude, will be

$$
5 \times 2 \pi r_{0} \sin 15^{\circ}=5 \times 10^{4} \text { amperes }
$$

All of this current must pass upward (or downward) along the field lines of the magnetospheric tail. Consequently, for any cross section through 


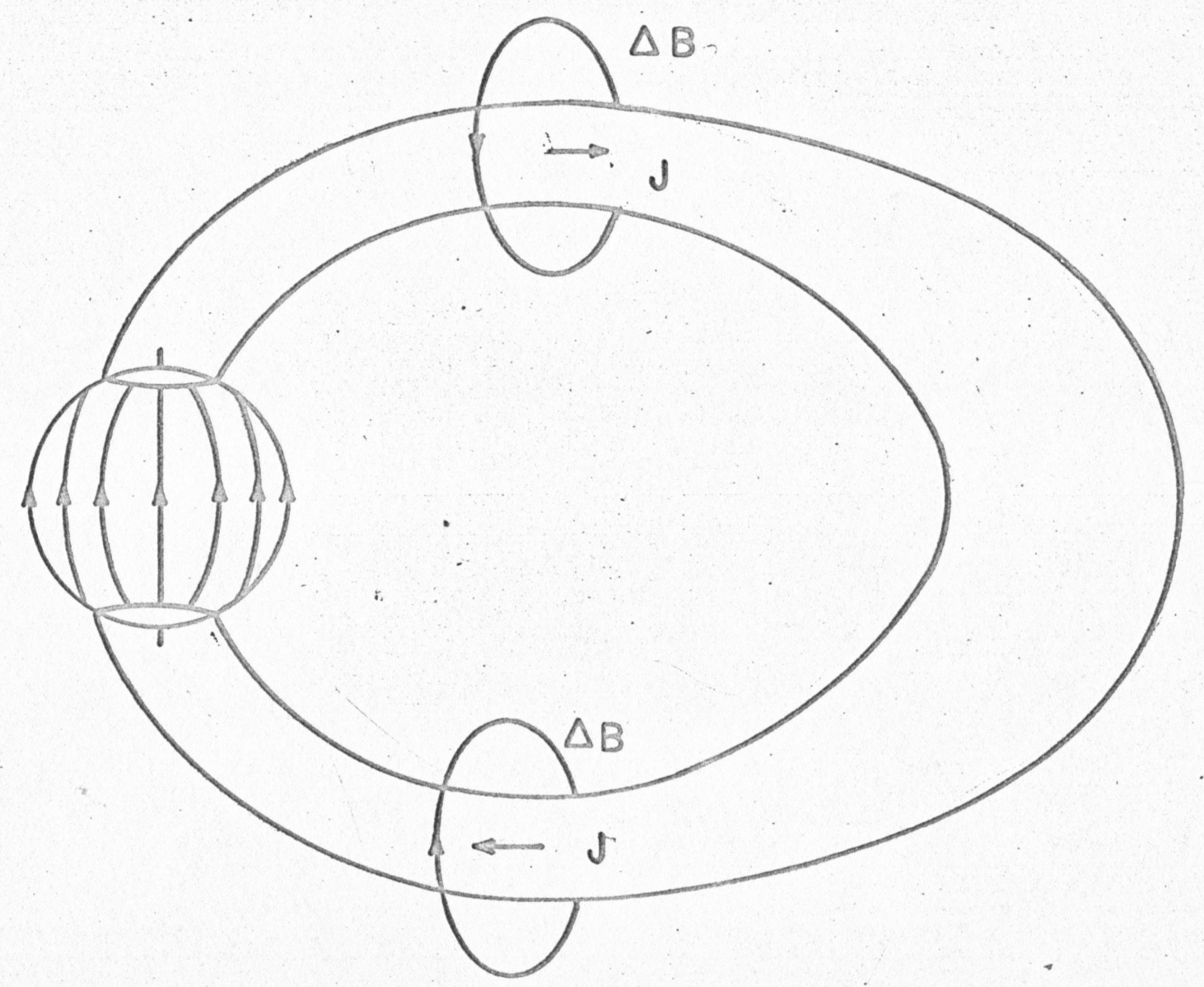

Fig. 5 Geometry for the estimate of the displacement of conjugate points caused by the distorting effect of an electric current from one hemisphere to the other along the polar cap field lines. 
the magnetospheric tail we must have

$$
\oint \underline{H} \cdot \underline{d s}=\int \nabla \times \underline{H} \cdot \underline{d a}=\int \underline{J} \cdot \underline{d a}=5 \times 10^{4} \text { amperes }
$$

where da is an elemental area of the cross section and the integral on the left is to be camied over the entire boundany of the cross section. We will assume that the cross section of the tail is circular with a radius of 10 earth radii and that the magnetic induction in the tail is $3 \times 10^{-8}$ webers $/ \mathrm{m}^{2}$ or 30 gammas. (This last quantity has been measured by Ness (1965) while the radius for the cross section is that which when combined with the 30 gammas field strength accounts for all the magnetic flux poleward of the $75^{\circ}$ latitude circle). Dividing $\oint \underline{H} \cdot d s$ by the circumference of the cross section of the tail and multiplying by the magnetic permeability of free space $\mu_{0}=4 \pi \times 10^{-7}$ henry/m, one arrives at a mean value for the perturbation of the magnetic field of the tail produced by the postulated $5 \times 10^{4}$ amperes of antisymmetric dynamo current. Thus

$$
\begin{aligned}
\Delta B=\mu_{0} \oint \underline{H} \cdot \underline{d S} / 2 \pi R & =1.6 \times 10^{-10} \text { webers } / \mathrm{m}^{2} \\
& =0.16 \text { gammas. }
\end{aligned}
$$

and so in addition to the 30 gammas magnetic induction along the tail, there would be a component around the tail of about 0.16 gammas, or about one part in 200. Consequently, if the magnetospheric tail has a length of 100 earth radii, the field lines near the surface will wrap around the tail about one half an earth radius, on put otherwise, the surface field will twist through about $3^{\circ}$ over its length. Such twisting would correspond to a displacement of the conjugate points of about $160 \mathrm{~km}$, 
this being the sum of the two antisymmetric displacements occurring in the two hemispheres. Other things being held constant, the displacement of the conjugate points will be directly proportional to the antisymetric cument, the length of the tail, and the radius of a cross section through the tail, and inversely proportional to the total magnetic flux through such a cross section of the tail. 


\subsection{Motions of the Magnetospheric Plasma Produced by Dynamo Action}

Many conjectures have lately been made regarding dynamic processes in the magnetosphere and motions of the magnetospheric plasma. (cf. Piddington (1960), Axford and Hines (1961) and Fejer (1964)). Aside from the motion of the plasma associated with the earth's rotation, most of these have placed the greatest emphasis on magnetospheric motions produced by forces having their origin in interplanetary space. It has also been recognized that the motion of the magnetospheric plasma is at least in part determined by the electrostatic field produced by the dynamo action of winds in the ionosphere. Hines (1963) has already made estimates of the flow patterns for the magnetospheric plasma produced by the superposition of the Sq electrostatic field on the electric field associated with the earth's rotation. The model for the electrostatic field he used is one suggested by Martyn (1955), obtained by assuming that the wind producing the $\mathrm{Sq}$ magnetic variation can be expressed in terms of a simple diumal wind potential of the form $\psi=\psi_{0} \sin ^{2} \theta \sin \lambda$ and that the conductivity can be adequately represented by considering the electron density uniform over the earth.

A better estimate of the electrostatic field associated with the current producing component of the symmetric wind may be obtained from the work of Maeda (1955). Using the surface harmonic analysis of the magnetic potential of the $\mathrm{Sq}$ field and a conductivity model that varies over the globe as a function of solar zenith angle $z$ of the form $(1+$ $2.00 \cos z+1.46 \cos ^{2} z$ ) he solved for the electrostatic potential and 
the wind velocity potential. It is the electrostatic potential which is here of interest to us. Neglecting the inertia of the plasma, the velocity $\mathrm{v}$ of the magnetospheric plasma is given by

$$
\underline{v}=\frac{-\nabla S \times \underline{B}}{B^{2}}
$$

where $S$ will be constant along the highly conductive magnetic field lines. Since - VS is perpendicular to the surfaces of constant $S$ and since $\underline{v}_{E}$ is perpendicular to both $\underline{B}$ and $-\nabla S$, the surfaces of constant $S$ intersect the surfaces orthogonal to $B$ to form the lines of flow for the velocity field $\mathrm{v}_{\mathrm{E}}$.

Maeda (1955) expresses the electrostatic field in terms of diurnally and semidiurnally varying vectors (or in our non-rotating point of view, vectors that undergo one and two cycles of variation over $360^{\circ}$ of longitude), given at $10^{\circ}$ increments of latitude. Let $E$ and $S$ be the electrostatic field and its potential due to the dynamo action. To derive the electrostatic potential the equation

$$
\underline{E}=-\nabla S
$$

can be expressed in spherical coordinates

$$
\begin{aligned}
& E_{\theta}(\theta, \lambda)=-\frac{1}{r_{0}} \frac{\partial}{\partial \theta} s(\theta, \lambda) \\
& E_{\lambda}(\theta, \lambda)=-\frac{1}{r_{0} \sin \theta} \frac{\partial}{\partial \lambda} s(\theta, \lambda)
\end{aligned}
$$

From (1.3) we can write

$$
S(\theta, 0)=-r_{0} \int_{0}^{\theta} E_{\theta}(x, 0) d x+S(0,0)
$$


where $x$ is the variable of integration. An estimate of the latitudinal variation of $S$ along the midnight meridian can be obtained by integrating by Simpson's rule using the given values of $E_{\theta}(\theta, 0)$. Equation $(1.4)$ can be integrated more directly:

$$
\begin{aligned}
s(\theta, \lambda) & =-r_{0} \sin \theta \int_{0}^{\lambda} E_{\lambda}(\theta, x) d x+s(\theta, 0) \\
& =r_{0} \sin \theta A_{1 y}(\theta)\left\{\cos \left(\lambda+\alpha_{2 y}(\theta)\right)-\cos \alpha_{1 y}(\theta)\right\} \\
& +\frac{1}{2} r_{0} \sin \theta A_{2 y}(\theta)\left\{\cos \left(2 \lambda+a_{2 y}(\theta)\right)-\cos \alpha_{2 y}(\theta)\right\} \\
& +s(\theta, 0)
\end{aligned}
$$

where $A_{2 y}(\theta), \alpha_{1 y}(\theta), A_{2 y}(\theta)$ and $\alpha_{2 y}(\theta)$ are given by Maeda (1955).

To the electrostatic potential derived above we must add the electrostatic potential associated with any other non-current producing ionospheric wind systems which we have reason to think exist. There is one such wind system of great importance, that associated with the earth's rotation. By wind we mean the rotational motion of the atmosphere that is observed when viewing the earth in a non-rotating reference frame, so what we are here referring to as a wind would, in the eyes of a rotating meteorologist, be considered a motionless atmosphere. The electrostatic potential associated with this motion is that which would exist if the "wind" dynamo electromotive force were everywhere just balanced by the electrostatic field. Thus, we have

$$
E_{R}=-\nabla S_{R}=-\underline{V}_{R} \times \underline{B}
$$


where $\underline{v}_{R}=\underline{1}_{\lambda} r_{0} \Omega \sin \theta$ and where $\Omega$ is the angular velocity of the earth's rotation. It is adequate for our purpose to consider only the $\theta$ component of the electrostatic field, since it is possible to derive the electrostatic potential from it alone. Thus the $\theta$ (southward) component of $E_{R}$ is

$$
E_{R \theta}=-v_{\lambda} B_{r}=r_{0} \Omega B_{p} \sin \theta \cos \theta
$$

where $B_{p}$ is a constant (about $6 \times 10^{-5}$ webers $/ \mathrm{m}^{2}$ ). Equating the above expression for $E_{R \theta}$ to the nonthward component of the gradient of the electrostatic potential and integrating from (the north pole) to any colatitude $\theta$, one obtains

$$
S_{R}=\frac{r_{0}^{2} \Omega B_{P}}{4} \cos 2 \theta+S^{\prime}
$$

where $S^{\prime}$ includes some constants of integration which can be taken to be zero.

Assuming for the moment that these are the major components of the electrostatic field, the mapping on the ionosphere of contours of constant electrostatic potential can be written

$$
S(0, \theta, \lambda)=S_{R}(\theta)+S(\theta, \lambda)
$$

The field lines through these contours generate surfaces of constant electrostatic potential in the magnetosphere along which the magnetospheric plasma flows.

Unfortunately, the longitudinal variation of the electrostatic potential is too small to enable the equipotential surfaces to be drawn from the functions evaluated at $10^{\circ}$ latitudinal increments as has been 
obtained above. However, an estimate of the flow lines can be made by means of a linear interpolution of the potential in the intermediate intervals: This has been done, resulting in Fig. 6 . The polar diagram corresponds to the north pole, with latitude shown on the radial coordinate and longitude, measured in hours from the midnight meridian, shown on the azimuthal coordinate. It must be remembered that these flow patterns are quite crude estimates of what might be expected to result from the dynamo action in the ionosphere. Two additional electrostatic field components of unknown configuration and magnitude are almost certain to exist. These are the electrostatic field associated with any undetected zonal current system and the electrostatic field associated with those parts of the wind velocity field that produce no current. Consequently, Fig. 6 should be considered an order of magnitude estimate of the deviation of the magnetospheric plasma flow from simple rotation.

\subsection{Role of Dynamo Action in Determining the Configuration of the Magnetosphere}

The magnetosphere is sometimes discussed in terms of a corotating region in which the magnetospheric plasma daily undergoes approximately one rotation about the earth, and a non-corotating region in which the plasma may still move concordantly from one field line to another, but the pattern of motion is such that streamines near the equatorial plane do not enclose the axis of the earth.

For the moment let us assume that the electric field in the magnetosphere arises entirely from the dynamo action associated with tidal motion and the earth's rotation. If this were the case, the surface bounding the corotating region, since it is by definition a stream surface (i.e. a surface on which lie stream lines of the magnetospheric plasma), must 


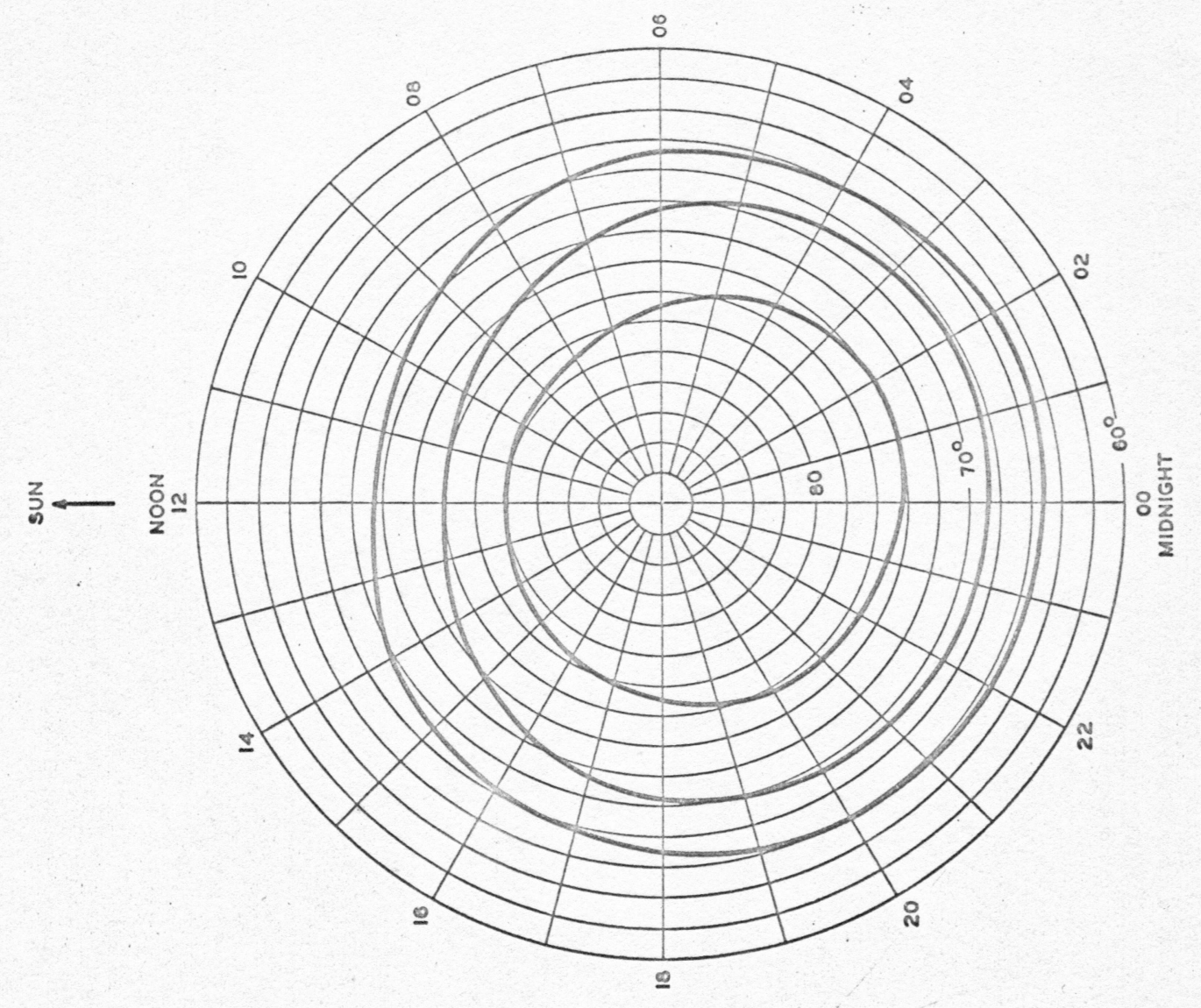

Fig. 6 Intersection of magnetospheric equipotential surfaces with the ionosphere. The equipotential surfaces represent the electric field obtained by combining the electrostatic field produced by tidal motions responsible for the production of the $\mathrm{Sq}$ magnetic variation

(Maeda, 1955) with that associated with the daily corotation of the magnetospheric plasma about the earth. 
correspond to a surface of equipotential of the dynamo induced electrostatic field. Consequently, any asymmetry of the dynamo induced electrostatic field will produce an asymmetry of the corotating region of the magnetosphere. In particular, the 5 to $10^{\circ}$ displacement of the equipotential sunfaces (see Fig. 6) toward the night side would cause the corotating region to show a contraction of about 5 to $10^{\circ}$ on the nightward side. Similarly, the streamines for the material in the noncorotating region would lie on other equipotential sunfaces which intersect the ionosphere at higher latitudes. Whether on not the asymmetry of the covotating region exceeds the 5 to 10 degrees produced by the dynamo action of atmospheric tides depends upon whether on not there exists additional electric fields of external origin. Any such electric field would produce ionospheric cuments and give rise to observable magnetic perturbations. Nagata and Kokubun (1962) report just such a magnetic perturbation, which they have designated $S_{q}^{p}$. The variation occurs most strongly in the sumer hemisphere, there having a magnitude of order 150 gammas in the $W$ component, or about 3 to 5 times that of the Low latitude Sq, and persists on even quite quiet days. The current system diagrams shown by Nagata and Kokubun suggest that the $S_{q}^{p}$ current system is largely confined to the polan region. If the electric field which drives the $S_{q}^{P}$ cunrent system is indeed entirely contained within the polar region, (i.e. the region connected to the non-corotating magnetosphere) the dynamo induced electric field might continue to be the most important influence in determining the configuration of the magnetosphere during magnetically quiet times. On the other hand, if the $S^{\text {? }}$ electric field extends into the conotating region, it would not only influence the conflgurction of the conotating region, but might also 
contribute to the low latitude $S_{q}$ current system, a current system now generally attributed solely to dynamo action. Thus, it is important to obtain a more thorough understanding of the magnetic variations in the polar regions, especially those that take place on the magnetically quietest days, in order to better understand the processes that shape the magnetosphere.

While it is difficult to determine just where the surface bounding the corotating region does intersect the ionosphere, there is some indication in the observations of energetic electrons trapped in the geomagnetic field. It has been observed by Freeman, Van Allen and Cahill (1963) that the outer limit of the region in which > $40 \mathrm{kev}$ electrons are observed near the equatorial plane corresponds closely to the outer boundary of the corotating region. Correspondingly, a poleward limit to the region where trapped $40 \mathrm{kev}$ electrons are found is observed at heights just above the ionosphere. A set of isointensity contours illustrating this poleward limit has been obtained by Frank, Van Allen and Craven (1964) and is shown in Fig. 7. There is considerable similarity between these isointensity contours and the equipotential surfaces based on Maeda's (1955) calculation of the dynamo induced electrostatic field. However, this similarity could be largely fortuitous, and should be interpreted with caution. Aside from the fact that Maeda's calculation does not give the entire electrostatic field, it is most important to point out that the equipotential surfaces are stream surfaces for oniy the thermal component of the magnetospheric plasma, that part which does not have an appreciable drift motion arising from the gradients of the magnetic field and curvature of the field ines. More enengetic particles (> $1 \mathrm{kev}$ ) will have an appreciable 


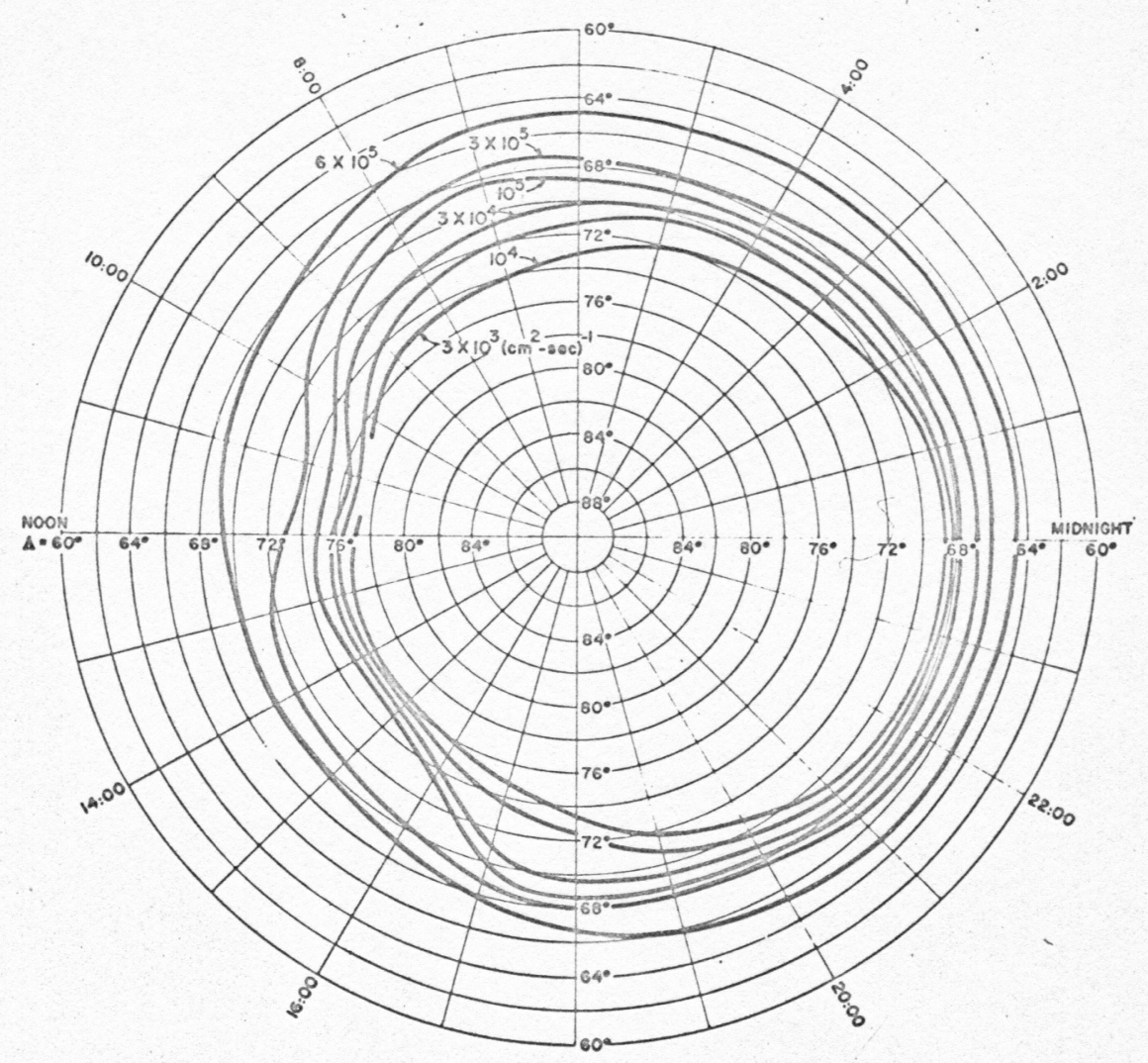

Fig. 7 Isointensity contours for trapped electrons with energies greater than $40 \mathrm{kev}$. Local time is plotted azimuthally and invariant latitude $(\Lambda)$ is plotted radially. The intensity of trapped radiation falls off rapidly with increased latitude, suggesting that the boundary of the corotating (inner) portion of the magnetosphere occurs near these contours. From Frank, Van Allen and Craven (1964). 
component of motion associated with the geometry of the magnetic field, and their total drift motion may be expected to follow paths departing appreciably from the equipotential surfaces.

In the absence of electric fields within the magnetosphere trapped particles of all energies would drift at different rates along surfaces of constant integral invariant $\int \sqrt{1-B / B_{m}}$ ds where the integral is taken between points where the particle mirrors). The angular velocity with which a panticle with energy W drifts along this surface is given by

$$
w_{a}=6 w r_{e} F(\alpha) /\left(0.32 \mathrm{er}_{0}^{3}\right)
$$

where $e$ is the charge of the particle, $r_{0}$ is the radius of the earth, and $r_{e}$ is the equatorial geocentric distance of the particles orbit, all in emu. The function of the particles equatorial pitch angle $\alpha$ is given by Hamlin et al. (1961) as approximately

$$
f(\alpha)=0.35+0.15 \sin \alpha .
$$

With appropriate quantities substituted, (2.1) becomes

$$
\omega_{a}=\left(r_{e} / 40 r_{0}\right) w \text { revolutions/day }
$$

where $w$ is in kev. Thus, $4 \approx 5 \mathrm{kev}$ particles at eight earth radii would drift around the surface of constant integral invariant about once per day.

Since the total drift velocity of particles will be the sum of the drift produced by the electric field and that determined by the configuration of the magnetic field, the degree to which a particle 
drifts along an equipotential surface, or along a surface of constant integral invariant may be estimated by comparing the magnitudes of the two drift velocities. The electric field drift is such as to produce one rotation per day about the earth. Consequently, particles trapped nean the boundary of the corotating region ( 8 earth radii) having energies much less than $5 \mathrm{kev}$ will follow the equipotential surfaces while particles with energies large compared to $5 \mathrm{kev}$ will tend to follow the surfaces of constant integral invariant.

It has been estimated by Malville (1960) that the distortion of the geomagnetic field by the solar wind might account for as much as a $2^{\circ}$ shift of the radiation belts toward the night side. This estimate receives support from Wilizams and Mead (1965) who discuss a nightward displacement of only 2.5 degrees for the isointensity contours of $>280 \mathrm{kev}$ electrons during magnetically quiet conditions. They further present a new calculation of the effect of field distortion based on the recent IMP satellite magnetometer observations of Ness (1964), which again predicts a displacement of only a couple degrees. Since $280 \mathrm{kev}$ electrons will be little influenced by electrostatic fields of the magnitude discussed here, the $2.5^{\circ}$ displacement observed by Williams and Palmer (1965) indicates the asymmetry of the surfaces of constant integral invariant. On the night side tho cut off occurs nean $67^{\circ}$, the same as that for the $40 \mathrm{kev}$ electrons. However, on the day side this limit occurs about 5 degrees equatorward of that reported for the 40 kev electrons. This, along with the observation in both cases that the cut off is more sharply defined on the night side, suggests that the poleward limit is actually established on the night side and the day side limit only represents the dayside position of the most poleward particle drift path 
that does not intersect the boundary of the region of stable trapping. Thus, if the poleward limit of the radiation belts on the night side corresponds to the boundary of the corotating region, the boundary on the day side can be expected to be somewhat poleward of the limit of trapped radiation (see Fig. 8 ).

We may now consider the question of whether the 5 degree greater nightward displacement of the $>40 \mathrm{kev}$ electron isointensity contours reported by Frank, Van Allen and Craven (1964) can be accounted for by the effects of dynamo induced electrostatic fields. Noting that $40 \mathrm{kev}$ electrons will drift around the earth about 8 to 9 times per day, it seems unlikely that the eight degree asymmetry of the equipotential surfaces can account for more than 10 to 20 per cent of their additional nightward displacement.

There are several possible interpretations of this discrepancy. First of all, there may be an additional electric field of external origin which gives rise to the unaccountable part of the nightward displacement of the $40 \mathrm{kev}$ trapped radiation. A second possibility is that the results of Frank, Van Allen and Craven (1964) are partly indicative of storm time conditions. In their analysis no separation of the data was made according to magnetic activity, whereas Williams and Palmer (1965) have found that even $280 \mathrm{kev}$ particles can show an additional 2 to 3 degrees nighward displacement under disturbed conditions. A third possibility is that the isointensity contours do not correspond to the ionospheric intersection of the surfaces along which $40 \mathrm{kev}$ electrons drift. This might be the case, for example, if acceleration processes were always taking place near the boundary of the corotating region, as suggested by O'Brien (1963). 
Since we are here largely concerned with the electric fields that exist in the magnetosphere, we will examine the first of the above mentioned possibilities, that of an electric field of origin other than dynamo action. In order to account for the displacement of the $40 \mathrm{kev}$ electron isointensity contours the electric field must be diurnal, and must be of sufficient horizontal scale to cover the polar cap down to about $60^{\circ}$ latitude. It must have a magnitude of 5 to 10 times that of the $\mathrm{Sq}$ field, and finally, it must arise from an accumulation of positive charge on the nightwand side in order to give rise to the observed nightward displacement of the trapped radiation.

Any electric field of external origin must give rise to currents in the ionosphere and to observable magnetic perturbations. At high latitudes there are three kinds of magnetic perturbation in addition to that arising from the dynamo action. These are the polar electrojet, the dayside agitation, and the quiet daily variation $S_{Q}^{P}$.

The intermittent nature of the polar electrojet immediately rules it out as a possible explanation for the nightward displacement of the $40 \mathrm{kev}$ electron radiation belt, unless the displacement itself is largely intermittent in nature. Assuming that the 6 to 7 degree displacement persists even during quiet times, the polar electrojet cannot account for the effect. Similarly, the dayside agitation is not sufficiently systematic to account for the displacement.

The $S_{q}^{P}$ magnetic variation would seem to be the only observational evidence of an electric field with many of the necessary characteristics. The $S_{q}^{P}$ current system deduced by Nagata and Kokubun (1962) is diurnal and covers the polar region down to about $65^{\circ}$ latitude. Furthermore, it is 

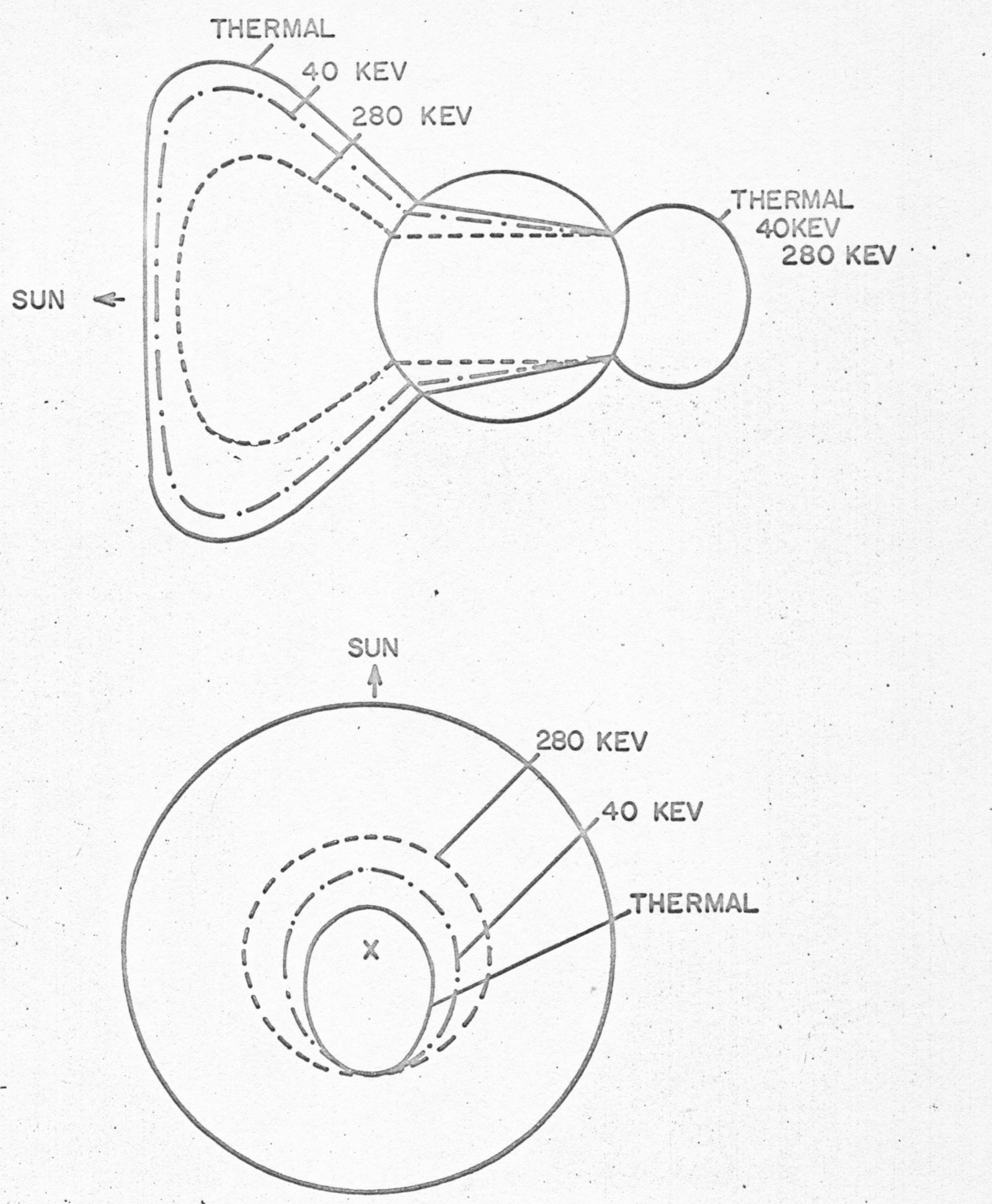

$\therefore x=$ GEOMAGNETIC POLE, 
Fig. 8 Relative positions of the boundaries of the regions in which $280 \mathrm{kev}$ particles, $40 \mathrm{kev}$ particles, and thermal particles are trapped in the magnetosphere. It has been assumed that the limit is imposed on the nightward side where the corotating region has been contracted as the result of day-night asymmetry in the magnetospheric electrostatic field. 
of the required onder of magnitude. The only discrepancy seems to be that if the current is to be considered largely a Hall current, the positive accumulation of charge associated with the $S_{q}^{p}$ current system is on the morning side rather than on the night side as would be required to explain the displacement of the radiation belts. 
APPENDIX A

DERIVATION OF THE CONDUCTIVITY OF A PARTIALLY IONIZED GAS, WITH THE EQUATION OF MOTION OF THE NEUTRAL CONSTITUENT INCLUDED

It is assumed here that the ionospheric and magnetospheric gas consists of three kinds of constituents, the neutral particles, positive ions and electrons. Their quantities are indicated by suffixes, $n, i$ and $e$, respectively. The gas is assumed to be neutral as a whole, so that $n_{e}=$ $n_{i}=n_{p}$; the suffix $p$ refers to the ionized gas. Then the equation of motion may be written for each constituent. Following Schluter (1951) and Watanabe (1957), we have

$p_{n} \frac{\partial v}{\partial t}+n_{n} n \alpha_{e n}\left(\underline{v}_{n}-\underline{v}_{e}\right)+n_{n} n_{p} \alpha_{i n}\left(v_{n}-\underline{v}_{i}\right)=-\nabla p_{n}$

$\rho_{e} \frac{\partial v_{e}}{\partial t}+n_{p}^{2} \alpha_{i e}\left(\underline{v}_{e}-\underline{v}_{i}\right)+n_{n} n_{p} \alpha_{e n}\left(\underline{v}_{e}-\underline{v}_{n}\right)=$

$$
-\nabla p_{e}-e n_{p}\left(\underline{E}+\underline{v}_{e} \times \underline{B}\right)
$$

$\rho_{i} \frac{\partial \underline{v}_{i}}{\partial t}+n_{p}^{2} \alpha_{i e}\left(\underline{v}_{i}-\underline{v}_{e}\right)+n_{n} n_{p} \alpha_{i n}\left(\underline{v}_{i}-\underline{v}_{n}\right)=$

$$
-\nabla p_{i}+e n p\left(\underline{E}+\underline{v}_{i} \times \underline{B}\right)
$$

where $p_{i}=m_{i}{ }^{n}$, $\rho_{e}=m_{e} e_{p}$ and $p_{n}=m_{n} n_{n}$. Here, the interaction between the neutral particles and the charged particles is expressed in terms of a sort of friction, so that it exerts a force which is proportional to the difference of their velocities, namely $\left(\underline{v}_{n}-\underline{v}_{e}\right)$ and $\left(\underline{v}_{n}-\underline{v}_{-i}\right)$; the coefficients of the proportionality are given by

$$
a_{i n}=v_{i n} \frac{m_{i} m_{n}}{p_{i}+p_{n}}
$$




$$
\alpha_{e n}=v_{e n} \frac{m_{e} m_{n}}{\rho_{e}+\rho_{n}}
$$

Similarly, the interaction between the positive ions and electrons are expressed by a Force which is proportional to $\left(\underline{v}_{i}-\underline{v}_{e}\right)$ with the coefficient

$$
\alpha_{i e}=v_{i e} \frac{m_{i} m_{e}}{p_{i}+p_{e}}=\frac{v_{i e}}{n_{p}} \frac{m_{i} m_{e}}{m_{i}+m_{e}}
$$

Introducing the following quantities,

$$
\begin{aligned}
& \underline{J}=\operatorname{en}_{p}\left(\underline{v}_{i}-\underline{v}_{e}\right)
\end{aligned}
$$

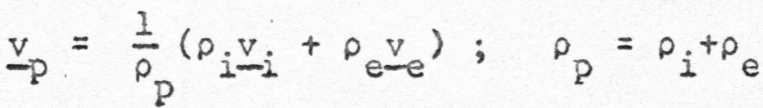

$$
\begin{aligned}
& \underline{v}=\frac{\rho}{\rho}\left(\rho_{n-n}+\rho_{p \rightarrow p}\right) ; \quad p=\rho_{n}+p_{p}
\end{aligned}
$$

and

$$
\underline{v}_{A}=\underline{v}_{-n}-\underline{v}=\frac{\rho}{p_{n}}\left(\underline{v}_{-p}-\underline{v}\right)
$$

we have

$$
\begin{aligned}
& \rho \frac{\partial \underline{v}}{\partial t}=\underline{J} \times \underline{B}-\nabla p \\
& p_{n} \frac{\partial v_{n}}{\partial t}=a\left(\underline{v}_{p}-v_{n}\right)+a b J-\nabla p_{n} \\
& -\rho_{p} \text { bo } \frac{\partial \underline{-p}}{\partial}+\frac{4 \pi \sigma}{\omega_{p}^{2}}\left(\frac{\partial J}{\partial t}\right)+\underline{J}= \\
& \sigma_{0}\left(\underline{E}+\underline{v} \times \underline{B}+b^{\prime} \underline{J} \times \underline{B}+\frac{n_{n} \alpha_{i n}}{e a} \nabla_{e}\right. \\
& -\frac{n_{n} \alpha_{e n}}{e a} \nabla p_{i}
\end{aligned}
$$


where

$$
\begin{aligned}
& a=n_{p} n_{n}\left(\alpha_{i n}+\alpha_{e n}\right) \\
& b=\frac{n_{n}\left(p_{e} \alpha_{i n}-p_{i} \alpha_{e n}\right)}{e a \rho_{p}} \\
& b^{\prime}=\frac{p_{e} \alpha_{e n}-\rho_{i} \alpha_{i n}}{p_{p}} \frac{n_{n}}{a e} \\
& \sigma_{0}=\frac{e^{2} a}{a \alpha_{i e}+n_{n}^{2} \alpha_{i n} \alpha_{e n}} \\
& \omega_{p}^{2}=4 \pi e^{2} \frac{p_{p}}{m_{i} m_{e}}
\end{aligned}
$$

We first assume a steady state exists, so all time derivatives are set equal to zero. Then (12) and (13) become respectively

$$
\begin{aligned}
& a\left(\underline{v}_{p}-\underline{v}_{n}\right)+a b \underline{J}-\nabla p_{n}=0 \\
& J=\sigma_{0}\left(\underline{E}+\underline{v}_{p} \times \underline{B}+b^{\prime} \underline{J} \times \underline{B}+\frac{n_{n} \alpha_{i n}}{e a} \nabla_{e}-\frac{n_{n} \alpha_{i n}}{e a} \nabla_{p_{i}}\right)
\end{aligned}
$$

which may be further rewritten as

$$
\begin{aligned}
\underline{J}=\sigma_{0}\left(\underline{E}+\underline{v}_{-n} \times \underline{B}\right. & +\left(b^{\prime}-b\right) \underline{J} \times \underline{B}+\frac{1}{a} \nabla p_{n} \times \underline{B} \\
& +\frac{n_{n} \alpha_{i n}}{e a} \nabla p_{e}-\frac{n_{n} \alpha_{e n}}{e a} \nabla p_{i}
\end{aligned}
$$

At this point we will drop the terms

$$
\frac{1}{a} \nabla p_{n} \times \underline{B}+\frac{n_{n} \alpha_{i n}}{e a} \nabla p_{e}-\frac{n_{n} a_{e n}}{e a} \nabla p_{i}
$$


only noting that they enter the equation in the same manner as E. Solving (17) for J gives

$$
\begin{aligned}
\underline{I}=\frac{\sigma_{0}}{1+\sigma_{0}^{2} B^{2}\left(b^{\prime}-b\right)^{2}} & \left(\underline{E}+\underline{v}_{-n} \times \underline{B}\right) \\
& +\frac{\sigma_{0}^{2}\left(b^{\prime}-b^{\prime}\right)}{1+\sigma_{0}^{2} B^{2}\left(b^{\prime}-b\right)^{2}} \underline{B} \times\left(\underline{E}+\underline{v}_{n} \times \underline{B}\right)
\end{aligned}
$$

This is of the form of the usual expression for current in a partially ionized gas

$$
\underline{J}=\sigma_{1}\left(\underline{E}+\underline{v}_{-n} \times \underline{B}\right)+\frac{\sigma_{2}}{B} \underline{B} \times\left(\underline{E}+\underline{v}_{n} \times \underline{B}\right)
$$

where the quantities in the place of conductivities are not quite the usual Pedersen $\left(\sigma_{1}\right)$ and Hall $\left(\sigma_{2}\right)$ conductivities. This is partly because our derivation has included the effect of collisions between charged particles.

Note that there is an inconsistency in this kind of derivation. We have dropped the pressure gradient terms and also the time derivatives. If all these terms were zero equation (11) would require the transverse current also to be zero. A similar step is made in most derivations of the Hall and Pedersen conductivities by assuming the neutral background immobile. Generally, the presence of a current indicates the presence of a pressure gradient, acceleration, or other kind of force. Let us then consider the effect of an acceleration of the neutral component (in the absence of pressure gradients) by allowing the electric field to vary as $e^{i \omega t}$. In the steady state all variables will undergo similar variations (although with different phases). Assuming this to be the case, (10) and (11) may be rewritten as 


$$
\begin{aligned}
& \underline{v}_{n}=\frac{a \underline{v}+a b \underline{J}}{i \omega p_{n}+a} \\
& \underline{v}=\frac{1}{i \omega \rho} \underline{J} \times \underline{B}
\end{aligned}
$$

Inserting (20) into (12) we have

$$
v_{p}\left(1-\frac{p_{n}}{p}+\frac{a p_{n}}{p\left(i \omega \rho_{n}+a\right)}\right)=v-\frac{a b p_{n} J}{p\left(i \omega p_{n}+a\right)}
$$

By use of (21) and (22), both $\underline{v}$ and $\underset{-p}{v}$ may be eliminated from (18) resulting in the following equation.

$$
\begin{aligned}
& \sigma_{0} \underline{E}=\underline{J}\left\{I+i \omega \frac{4 \pi \sigma_{0}}{\omega_{p}^{2}+i \omega \frac{\rho_{p} p_{n} a b^{2} \sigma_{0}}{i \omega \rho_{n} \rho_{p}+a p}}\right\} \\
& -\underline{J} \times \underline{B}\left\{b^{\prime} \sigma_{0}+\frac{\rho_{p} b_{0}\left(i \omega \rho_{n}+a\right)-\sigma_{0} a \rho_{n}}{\left(i \omega \rho_{p} \rho_{n}+a p\right)}\right\} \\
& -(\underline{J} \times \underline{B}) \times \underline{B} \frac{\sigma_{0}\left(i \omega \rho_{n}+a\right)}{i \omega\left(i \omega p_{p} p_{n}+a_{p}\right)}
\end{aligned}
$$

Assuming $\underline{E}$ is perpendicular to $\underline{B}$

$$
\underline{E}=\underline{P J}-Q(\underline{J} \times \underline{B})
$$

where

$$
\begin{gathered}
P=\frac{1}{\sigma_{0}}\left\{1+i \omega \frac{4 \pi \sigma_{0}}{\omega_{p}^{2}}+\frac{i \omega p_{n} a b^{2} \sigma_{0}}{i \omega p_{n} \rho_{p}+a_{p}}+\frac{\left(i \omega \rho_{n}+a\right) \sigma_{0}}{i \omega\left(i \omega \rho_{p} \rho_{n}+a p\right)}\right\} \\
Q=b^{\prime}+\frac{p_{p} b\left(i \omega \rho_{n}+a\right)-a b \rho_{n}}{\left(i \omega \rho_{p} p_{n}+a p\right)}
\end{gathered}
$$

and $P$ and $Q$ are complex numbers. 
In terms of the $x$ and $y$ components, (24) are

$$
\begin{aligned}
& \text { Q }=Q B \\
& P J^{x}-Q J^{x}+P J^{y}=E^{y}
\end{aligned}
$$

or rewriting (27)

$$
\begin{aligned}
& J_{x}=Y_{1} E_{x}+Y_{2} E_{y} \\
& J_{y}=-Y_{2} E_{x}+Y_{1} E_{y}
\end{aligned}
$$

where

$$
\begin{aligned}
& Y_{1}=\frac{P}{P^{2}+Q^{\prime 2}} \\
& Y_{2}=\frac{Q^{\prime}}{P^{2}+Q^{2}}
\end{aligned}
$$

Here, $Y_{1}$ is the generalized Pedersen conductivity and $Y_{2}$ is the generalized hall conductivity. Since these quantities are complex numbers, they correspond to the admittance used in a.c. circuit analyses. The conductivities $Y_{1}$ and $Y_{2}$ should be reduced to $\sigma_{1}$ and $\sigma_{2}$, respectively, when the neutral particles are considered to be an immobile background.

The conductivity along the field lines can also be obtained from (23);

$$
J_{11}=Y_{0} E_{11}
$$

where

$$
Y_{0}=\frac{1}{\frac{1}{\sigma_{0}}+\left(\frac{4 \pi}{\omega_{p}^{2}}+\frac{p_{p} p_{n} a b^{2}}{i \omega p_{n} p_{p}+a p}\right) i \omega}
$$


Equation (3) indicates that the important parameters which determine the efficiency of transmission of the electric field is $\left(Y_{1} / Y_{0}\right)$. Recalling that we have assumed a periodic change $\left(e^{i \omega t}\right)$ for the electric field, let us take the periods $10 \mathrm{sec}, 100 \mathrm{sec}, 6 \mathrm{hr}, 8 \mathrm{hr}, 12 \mathrm{hr}$ and $24 \mathrm{hr}$ for the calculation of the conductivities. In Figs. Al - A3 the distribution of the generalized Pedersen conductivity $Y_{1}$ (esu) is shown as a function of altitude $(60 \mathrm{~km} \sim 500 \mathrm{~km})$. In general, $Y_{1}$ is a complex number, so that the absolute values of the real part $(R)$ and the imaginary part (I) are given separately, together with the parameter 'period'. The imaginary part has a negative value below $100 \mathrm{~km}$, but this does not have a significant effect because $R>I$. The curves $R-10 S$ and $I-10 S$ in Fig. Al refer to the real and imaginary parts of $Y_{1}$ for the period of $10 \mathrm{sec}(S)$, and similarly, the curves $R-6 H$ and $I-6 H$ in Fig. A2 refer to the real and imaginary parts of $y_{1}$ for the period of 6 hour $(H)$, respectively. In all the figures, the distribution of $\sigma_{1}$ calculated by use of the usual expression is also included.

First of all, for short period changes (periods $<10^{3} \mathrm{sec}$ ) of the electrostatic field, the real part of $Y_{1}$ is essentially the same as $\sigma_{1}$. However, beyond $100 \mathrm{~km}$ the imaginary part increases rapidly with altitude. It is not difficult to show that the imaginary part in this period range is due to the polarization current $J_{p}$ namely

$$
J_{p} \simeq i \omega p_{p} \frac{E}{B^{2}}=\left(\frac{P_{p}}{B^{2}}\right) \frac{\partial E}{\partial t}
$$

This current does not exist in a steady state $(\partial / \partial t=0)$, so that the usual expression for $\sigma_{2}$ does not contain the contribution from the polarization current. 
Fig. A The complex conductivity (admittance) of the ionosphere in the direction of a transverse electric field which varies sinusoidally. The imaginary part of the conductivity arises from the tendency of the neutral component of gas to be accelerated by collisions with the ionized components. Fig. Al shows the real. (R) and imaginary (I) conductivities for applied electric fields with 10 and 100 second periods: Fig. A2, for 6 and 8 hour periods and Fig. A3, for 12 and 24 hour periods. 


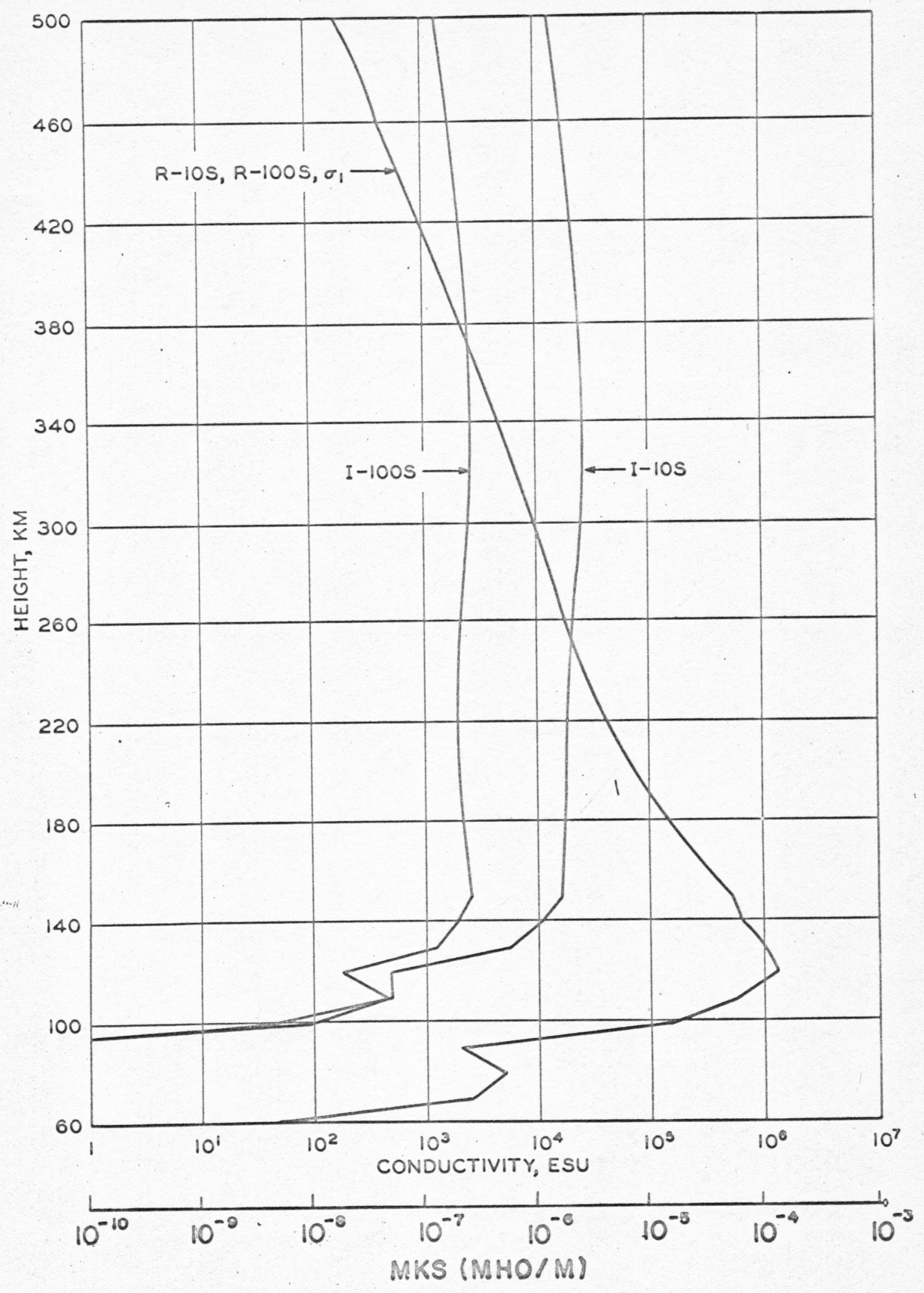



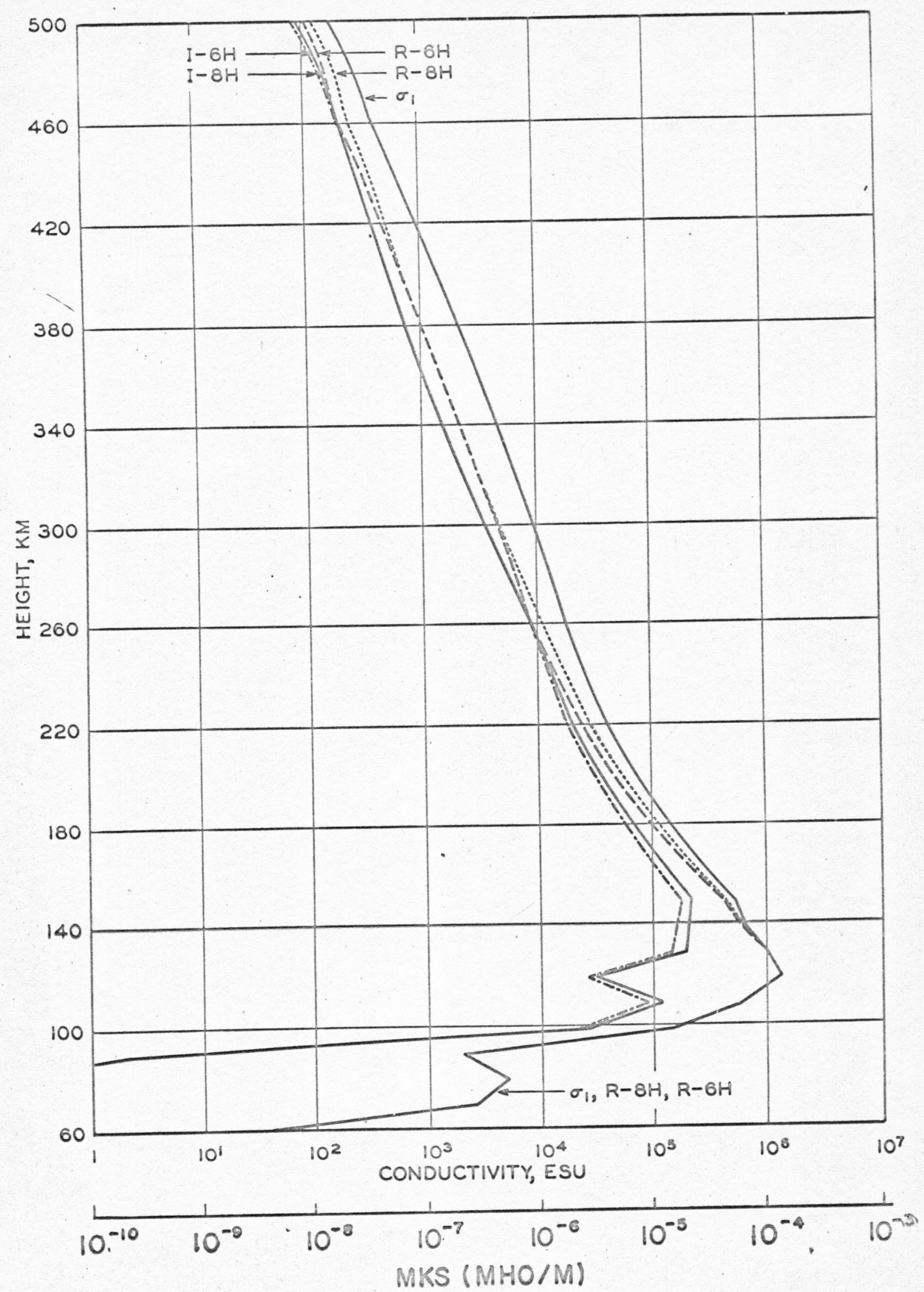


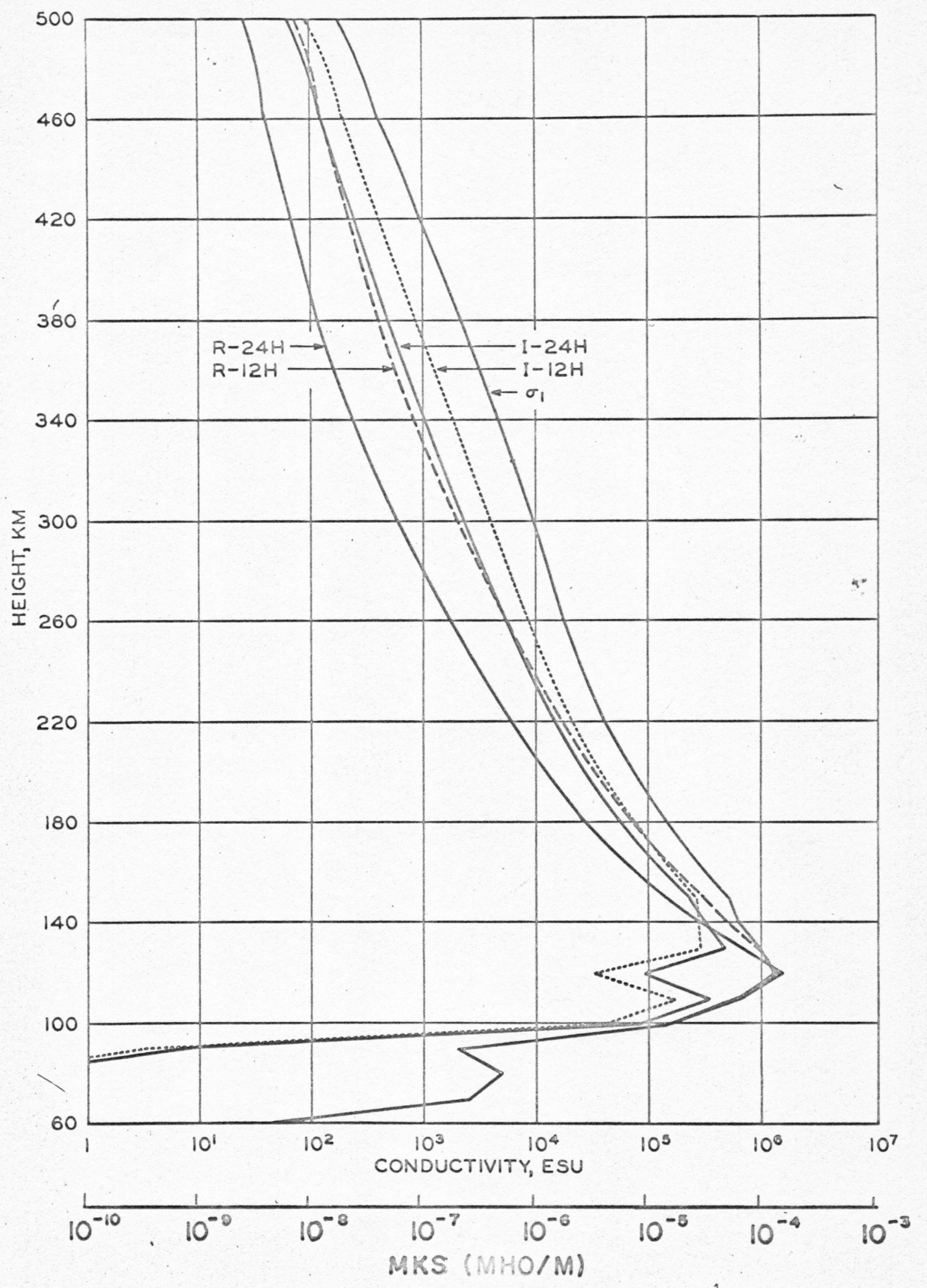


Secondly, below $100 \mathrm{~km}, Y_{1}$ is essentially the same as $\sigma_{1}$ for all the periods we consider. This is because the neutral particles are so abundant in that region that they cannot respond to the applied field, and they essentially behave as an immobile background.

However, for a period longer than 6 hrs, the real part of $Y_{1}$ becomes less than $\sigma_{1}$ beyond $150 \mathrm{~km}$ in altitude. The discrepancy becomes more serious for longer periods. For a period of $24 \mathrm{hrs}$, the real part of $Y_{1}$ is more than order of magnitude less than $\sigma_{1}$.

This discrepancy can be considered to arise from the interaction between the charged particles and the neutral particles. The usual derivation of the conductivities assumes that the charged particles, after an interval of acceleration by the applied electric field, undergo collisions with neutral particles of a gas with zero mean velocity. However, after a number of such collisions sufficient momentum would be systematically transferred to the neutral particles to give them a significant mean velocity, causing a change in the character of their collisions with the charged particles. It is the last effect that produces the discrepancy between the results given here and those previously obtained. It can be noted that so long as the inertia of the neutral gas is great enough to prevent it from responding to a given frequency of the applied electric field, the results obtained here are not unlike those previously obtained. If the gas can be accelerated appreciably during the time of one cycle of the applied field, however, there is an appreciable change in the effective conductivity.

The effect of acceleration of the neutral component of the gas is equally well treated in terms of dynamo effect. This treatment essentially employs a coordinate system which moves at the gas velocity, insuring that 
the usually used conductivities are appropriate. The reduction of the generalized Pedersen conductivity can be demonstrated in this way. From equation (11) the mass velocity $\underline{v}$ of the whole gas is given by

$$
\underline{v}=\underline{J} \times \underline{B} / i \omega_{0}
$$

Let $E$ be an applied electric field in the upper ionosphere. In the upper ionosphere, the Pedersen current becomes more important than the Hall current. Therefore, let us assume that the current equation is approximately given as follows:

$$
\sigma_{1}(\underline{E}+(\underline{V} \times \underline{B}))=\underline{J}
$$

or

$$
\sigma_{I}\left(\underline{E}-\frac{B^{2}}{i \omega p} \underline{J}\right)=\underline{J}
$$

and thus

$$
I=\left(\frac{\sigma_{1}}{1+\frac{\sigma_{1}^{2} B^{4}}{\omega^{2} \rho^{2}}}+i \frac{\frac{\sigma_{1}^{2} B^{2}}{\omega \rho}}{1+\frac{\sigma_{1}^{2} B^{4}}{\omega^{2} \rho^{2}}}\right) E
$$

If the angular frequency $\omega$ is high enough, (34) can be reduced to

$$
J=\sigma_{1} \underline{E}
$$

For a sufficiently low frequency change (or a sufficiently long period),

$$
\frac{\sigma_{1}^{2} B^{4}}{\omega^{2} \rho^{2}}>1\left(\text { or } \sigma_{1}>\frac{\omega \rho}{B^{2}}\right)
$$


so that the real part in the bracket is less than $\sigma_{1}$ and the imaginary part is given approximately by

$$
\frac{\omega p}{B^{2}}
$$

This situation is approximately attained above the F2 peak for periods of order 24 hrs. For an extremely low frequency, both the real and imaginary parts become null; this situation is given by

$$
\underline{E}+(\underline{v} \times \underline{B})=0
$$

It is important to realize that an electrostatic field transmitted from the magnetosphere (or from the dynamo effective region of the ionosphere) to the upper ionosphere can cause a wind of the neutral gas there (Martyn $(1954)$ )). The fact that $Y_{1}$ is significantly less than $\sigma_{1}$ for periods of more than 6 hrs suggests that the wind attained a speed $v \simeq v_{n}$ comparable to $(E / B)$.

The results for $Y_{0}$ is much simpler than those for $Y_{1}$. It can easily be shown that in the circumstance discussed above

$$
Y_{0} \approx \sigma_{0}(\sec 14 d)
$$

which is a little more general than the usual expression for $\sigma_{0}$, in that the collisions between the positive ions and electrons are taken into account. Note that $\sigma$ can be reduced to the well known conductivity in a fully ionized gas $e^{2} n_{p} / m_{e} v_{i e}$, depending on whether $a \alpha_{i e}>n_{n}{ }^{2} \alpha_{i n}$ or $\alpha \alpha_{i e}<n_{n}^{2} \alpha_{i n} \alpha_{e n}($ see equation $(14 \alpha))$. 


\section{APPENDIX B \\ CONDITIONS FOR THE EXISTENCE OF TURBULENCE AT THE LOWER THRESHOLD OF THE DYNAMO REGION}

It has been observed that turbulence exists just below the dynamo layer, but never above a height somewhere between 100 and $105 \mathrm{~km}$. We here wish to consider the circumstances under which such turbulence can exist under the assumption that it is driven by a velocity shear arising from the inability of the dynamo layer to undergo the same motion as the upper portion of the neutrosphere. We will make the further assumption that inertial and pressure terms are not important, so we may consider each layen to be in dynamic equilibrium under the stress exerted on it at its lower surface by the underlying layer, and that at its upper surface exerted by the layer above. Under these circumstances it immediately follows that the stress must be constant on all horizontal surfaces.

There are three means by which stress may be exerted on overlying and underlying surfaces. The first is the usual molecular viscosity. The second process, "eddy viscosity", is a much more efficient one, given the existence of turbulence. Finally, there is a process which normally does not enter into such discussions, that arising from the Lorentz force $\mathrm{J} \times \mathrm{B}$. In this form the Lorentz force is a force on each unit volume of material rather than a stress, but it can easily be put into the form of a stress. First, however, let us consider the nature of the reaction to the force. Consider a single elemental layer in the vicinity of $100 \mathrm{~km}$, and divide the horizontal current in that layer into two parts, a solenoidal part and a part which is irrotational. The irrotational part of the horizontal current will have divergence, and consequently in the steady state a vertical current will exist in order 
to meet the condition that the three dimensional current be divergence free. However, since we cannot have vertical currents to great distances, the net divergence of all other layers must be equal and opposite to that of the elemental layer we are discussing. We may speak of the net current of all the other layers, and this will be equal and opposite to that of this elemental layer. Thus, we find that an equal and opposite force is distributed in some fashion among all other layers as a reaction to the Lorentz force associated with the irrotational current in a particular layer. The Lorentz force associated with the solenoidal component of current will similarly have a reaction in all other layers. This time, however, the currents associated with the reaction force may be considered to be induced by the perturbation of the magnetic field produced by the current in the elemental layer under discussion. In both cases the effect is the same. A force introduced into the layer by some other process is transferred to other layers by electromagnetic action. Thus, in our case, the Lorentz force in a particular layer must be associated with a change with height in the viscous stresses. We may write

$$
\underline{J} \times \underline{B}+\frac{\partial}{\partial z} \underline{I}=0
$$

If we integrate upward from some height below that where the atmosphere has electrical conductivity to any height $z$, we have

$$
\int_{z_{0}}^{z} \underline{J} \times \underline{B} d z+\underline{\tau}=\tau_{0}
$$

where $\tau_{0}$ is the viscous stress at heights below those at which the Lorentz force can act. Let us begin by considering the quite simple 
case in which all the stresses are in the same direction. This would be the case if the conductivity were expressible as a scalar and if there were no electric field. Disregarding the turbulent component of motion we have

$$
\underline{J}=0 \underline{v} \times \underline{B}
$$

and we may write (2) in terms of a single component of stress as

$$
\tau_{E}=\tau_{0}-B^{2} \int_{z_{0}}^{z} \sigma \vec{v} d z-\tau_{m}
$$

where we have divided the viscous stress into an eddy viscous $\left(\tau_{E}\right)$ and a molecular viscous $\left(\tau_{m}\right)$ part. The eddy viscous stress can be related to the velocity shear by the relationship

$$
\tau_{E}=\rho I_{m}^{2}\left(\frac{\partial \bar{v}}{\partial z}\right)^{2}
$$

where $I_{m}$ is the Prandtl mixing length.

In order to sustain turbulence the kinetic energy of the flow must do work on the atmosphere, transporting parcels of the atmosphere in opposition to the stabilizing effects of gravity. This condition is expressed in terms of the dimensionless Richardson number $R i$

$$
R i=\left(g \frac{\partial}{\partial z} \ln \bar{\theta}\right) /\left(\frac{\partial \bar{v}}{\partial z}\right)^{2}
$$

where $g$ is the acceleration of gravity, $\bar{\theta}$ is the average potential temperature, and $\bar{v}$ is the average velocity of the flow. In order for turbulence to be sustained this number must be less than some limiting value in the vicinity of 1 . The precise value of this limiting value 
is not well established, and we will here simply denote it by Rimax.

This places a condition on the magnitude of the wind shear necessary to sustain the turbulence, namely, that

$$
\frac{\partial v}{\partial z}>\left[\left(g \frac{\partial}{\partial z} \ln \bar{\theta}\right) / \operatorname{Rimax}\right]^{\frac{1}{2}}
$$

Another commonly used criteria for the existence of turbulence is the Reynolds number

$$
R_{e}=v d p / \mu
$$

where d a dimension characteristic of the flow, $\rho$ is the mass density and $\mu$ is the molecular viscosity of the fluid. The characteristic velocity $\checkmark$ must be associated with the shear (since a uniform flow can be generated in the choice of a reference frame which can hardiy be influential in producing turbulence) allowing us to write

$$
R_{e}=\frac{\partial \bar{v}}{\partial z} d^{2} \rho / \mu
$$

The Reynold's number may be identified with the ratio of the inertial force $p\left(\bar{v} \frac{d \bar{v}}{d z}\right)$ to the viscous force. In order to have turbulence the Reynold's number must exceed some minimum value which we will here denote Remin. Again, this number is not well known and suggestions in the literature for its critical value range from one to two thousand. Nevertheless, we will suppose some such minimum number must be exceeded to maintain turbulence, and rewrite this condition as an inequality

$$
\frac{\partial \bar{v}}{\partial z} d^{2}>(\mu / \rho) \text { Remin }
$$


The $d$ which appears in the inequality (10) and the Prandtl mixing length appearing in equation (5) may both be regarded as measures of the scale of the eddies. Thus, if we multiply inequality (7) by (10) and substitute $I_{m}$ for $d$ we obtain

$$
\tau_{E}=\rho l_{m}^{2}\left(\frac{\partial \bar{v}}{\partial z}\right)^{2}>\left[\left(g \frac{\partial}{\partial z} \ln \bar{\theta}\right) / \operatorname{Rimax}\right]^{\frac{1}{2}} \mu \operatorname{Remin}
$$

which places a lower limit on the stress which must exist in order to sustain turbulence. But by (4) we find that this places an upper limit on the electromagnetic and moleculan viscous coupling that can take place before turbulence is suppressed.

$$
\mu \frac{\partial \vec{v}}{\partial z}+B^{2} \int_{z_{0}}^{z} \sigma \bar{v} d z<\tau_{0}-\left\{\left(g \frac{\partial}{\partial z} \ln \bar{\theta}\right) / \operatorname{Rimax}\right\}^{\frac{3}{2}} \mu \operatorname{Remin}
$$

It is interesting to note that the integral on the left does not characterize any one layer, but is rather the accumulation of effects at all levels below $z$, the level under discussion. Consequently, when an altitude is reached at which this condition would deny the existence of turbulence, the existence of turbulence would be denied at greater heights as well, unless new stresses are introduced in some manner. A second interesting point concerning the integral, indeed one that demands explanation, is that it involves the velocity $v$ rather than a velocity shear. This implies that the reference frame in which the flow is observed has somehow been established in the course of our arguments. This is indeed the case, for in writing equation ( 3 ) we assumed there is no electric field. If an electric field is present, it is necessary to transform the problem to a coordinate system moving 
with velocity $\underline{V}_{E}=\underline{E} \times \underline{B} / B^{2}$ if these results are to be applied, or to replace $\underline{v}$ by $\underline{v}-\underline{v}_{E}$ in the development. This requirement regarding the choice of coordinate systems is indicative of the fact that the stress is being applied to those layers whose electric field is influenced by the dynamo action of other layens. We note that if $\underline{v}=\underline{v}$ there would be no current in the level under discussion and no transfer of force by electromagnetic effects.

At sufficiently great heights in the ionosphere the conductivity along the magnetic field lines is sufficiently great that they may be regarded as equipotentials and the eiectric field is essentially uniform with height. We are here concerned with the threshold of the dynamo region, so the assumption that the same electric field exists at all heights warrants some examination. Appreciable electrical resistance along the field lines would to some extent allow an electric polarization to be established in a layer independent of the electric field in more distant layers. Such a polarization would act to inhibit the current, thus reducing the Lorentz force on the layer. Effectively, the resistance along the field line serves to decouple the layers. Some estimate of this decoupling action may be obtained using an argument similar to that which was made in Chapter 4, where the relative importance of transverse currents and currents along the field lines were discussed for the case of antisymmetric dynamo action.

Consider a surface, assumed motionless and equipotential for simplicity, just above a region of thickness $z$ in which there is a more or less uniform vertical velocity shear. Let us assume that in this region an electrostatic field has been established by the wind's dynamo action. We wish to know whether this electrostatic field drives currents 
predominantly transverse to the magnetic field (here assumed vertically directed), thus acting to cancel the Lorentz force on the gas, or predominantly upward, which would amount to dynamic coupling to overlying layers. Consider a region of horizontal scale $L$ which is at a higher potential than the surrounding. We further suppose it to be surrounded by a second region of horizontal scale $2 \mathrm{~L}$ which is at zero potential. From equation (1.8) of Chapter 4 we have for the resistance between the two regions

$$
R_{\text {disc }}=2 \pi \cdot 2 /\left(2 \pi \mathrm{Z}_{1}\right)
$$

The resistance between our reference surface and the region of higher potential is

$$
R_{\text {tube }}=2 /\left(\sigma_{0} \pi L^{2}\right)
$$

Thus, the current along the field lines will be greater than that transverse to the field so long as

$$
R_{\text {tube }}<R_{\text {disc }}
$$

or

$$
Z /\left(\sigma_{0} \pi L^{2}\right)<\ln 2 /\left(2 \pi Z \sigma_{1}\right)
$$

Solving the inequality for $Z$ we have

$$
z<\frac{\ln 2}{2}\left(\frac{\sigma_{0}}{\sigma_{1}}\right)^{\frac{1}{2}}
$$

In general $\sigma_{0}>\sigma_{1}$ at all heights in the ionosphere. Thus, for fields having appreciable horizontal scale $(>100 \mathrm{~km})$ there seems to be little likelihood that the resistance along the field lines would be effective in decoupling layers in which there are dynamo driven transverse cunrents. 


\section{APPENDIX C}

THE DIVISION OF A HORIZONTAL IRROTATIONAL WIND INTO

CURRENT PRODUCING AND NON-CURRENT PRODUCING PARTS

In order to determine $S_{1}$ and $\psi$ such that

$$
B_{r} \underline{v}=-B_{p} \cos \theta \nabla \phi=-\nabla \times S_{1} \underline{1}_{r}-\nabla \psi
$$

on the spherical shell $r=r_{0}$ we first take the divergence of both sides of (1). This gives

$$
\begin{aligned}
\nabla_{\psi}^{2} & =B_{p}\left(\nabla \phi \cdot \nabla \cos \theta+\nabla^{2} \phi \cos \theta\right) \\
& =B_{p}\left(\nabla^{2} \phi \cos \theta-r^{-2} \sin \theta \frac{\partial \phi}{\partial \theta}\right),
\end{aligned}
$$

a differential equation for $\psi$. Next taking the curl of both sides of (1) one obtains

$$
\nabla \times\left(\nabla \times S_{1}{ }_{-r}\right)=B_{p} \nabla \times(\cos \theta \nabla \phi)
$$

which may be rewritten

$$
I_{r}\left(B_{p} / r^{2}\right) \frac{\partial \phi}{\partial \lambda}=I_{r} \nabla^{2} S
$$

making use of the fact that $S_{1}$ and $\phi$ have only horizontal gradients. This is then the required differential equation for $S_{1}$. 
Adams, J. C., Useful formulae connecting Legendre's coefficients, which are employed in the theory of terrestrial magnetism, with extensive tables, Scientific Papers, 2, 243-400, Camb. Univ. Press, 1900.

Alfvén, H., Cosmical Electrodynamics, Oxford Univ. Press, 1950.

Axford, W. I. and C. O. Hines, A unifying theory of high latitude geophysical phenomena and geomagnetic storms, Can. J. of Phys., 39, 1433-1464, 1961.

Baker, W. G., Electric currents in the ionosphere II. The atmospheric dynamo, Phil. Trans. Roy. Soc. A, 295-305, 1954.

Baker, W. G. and D. F. Mantyn, Electric currents in the ionosphere, Phil. Trans. Roy. Soc. A, 246, 218-294, 1954.

Batchelor, G. K. and R. M. Davies, Surveys in Mechanics, Camb. Univ. Press, 1956.

Blamont, J. E. and C. de Jager, Upper atmospheric turbulence near the $100 \mathrm{~km}$ level, Ann. Geophys., 17, 134-144, 1961.

Butler, S. T. and K. A. Small, The excitation of atmospheric oscillations, Proc. Roy. Soc. A, 274, 91-121, 2963.

Chandrasekhar, S., Plasma Physics, Univ. of Chicago Press, Chicago, 1960.

Chandrasekhar, S., Hydrodynamic and Hydromagnetic Stability, Clarendon Press, Oxford, 1961.

Chapman, S., On the diurnal variations of the earth's magnetism produced by the moon and sun, Phil. Trans. London (A), 213, 279-331, 1913.

Chapman, S., The lunar diurnal magnetic variation and its change with lunar disturbance, Terr. Mag., 23, 25-28, 1918.

Chapman, S. and J. Bartels, Geomagnetism, Oxford Univ. Press, 1940.

Chapman, S. and V. C. A. Ferraro, A new theory of geomagnetic storms, Terr. Mag., 36, 77-97, 1931; 171-185, 1931.

Cole, K. D., Damping of magnetospheric motions by the ionosphere, J. Geophys. Res., 68, 3231-3235, 1963.

Dougherty, J. P., Some comments on dynamo theory, J. Geophys. Res., 68, $2383-2384,1963$.

Fejer, J. A., Semidiumal currents and electron drifts in the ionosphere, J. Atmospheric Terrest. Phys., 4, 184-203, 1953. 
Fejer, J. A., Theory of the geomagnetic daily disturbance variations, J. Geophys. Res., 69, 123-137, 1964.

Eerraro, V. C. A., The non-uniform rotation of the sun and its magnetic field, Mon. Not. R.A.S., 97, 458-472, 1937.

Frank, L. A., J. A. Van Allen and J. D. Craven, Large diumal variations of geomagnetically trapped and of precipitated electrons observed at low altitudes, J. Geophys. Res., 69, 3155-3167, 1964.

Freeman, J. W., J. A. Van Allen and L. J. Cahill, Jr., Explorer 12 observations of the magnetospheric boundary and the associated solar plasma on September 13, 1961, J. Geophys. Res., 68, 2121-2130, 1960.

Gold, T., Motions in the magnetosphere of the earth, J. Geophys. Res., 64, $1219-1224,1959$.

Hamlin, D. A., R. Kamplus, R. C. Vik and K. M. Watson, Mirror and azimuthal drift frequencies for geomagnetically trapped particles, J. Geophys. Res., 66, 1-4, 1961,

Hasegawa, M. and M. Ota, On the magnetic field of $\mathrm{Sq}$ in the middle and lower latitudes during the second polar year, Trans. Oslo Meeting, IATME BU11. No. 13, 426-434, 1948.

Haurwitz, B. and G. M. Sepulveda, J. Meteorol. Soc. Japan, 75th Anniversary, Vol. 149, 1957.

Hines, c. O., Motions in the ionosphere, Proc. Inst. Rad. Engr., 47, $176-186,1959$.

Hines, C. O., Geomagnetism and the ionosphere, Proc. Intern. Conf. on the Ionosphere, Imperial College, London, 103-115, July 1962.

Hines, C. O., The upper atmosphere in motion, Quart. J. Roy. Met. Soc., 89, 1-42, 1963.

Hines, C. 0., Hydromagnetic motions in the magnetosphere, Space Science Reviews, $\underline{3}, 342-379,1964$.

Hirono, M., A theory of diurnal magnetic variations in equatorial regions and conductivity of the ionosphere E region, J. Geomag. Geoelec., 4. $7-21,1952$.

Jacchia, I. G. and Z. Kopal, Atmospheric oscillations and the temperature profile of the upper atmosphere, 3. Met., 9, 13-23, 1952.

Kato, S., Horizontal wind systems in the ionospheric E region deduced from the dynamo theory of the geomagnetic $S_{q}$ variation, J. Geomag. Geoelec., 8, 24-37, 1956.

Kochanski, A., Atmospheric motions from sodium cloud drifts, J. Geophys. Res., 69, (17), 3651, 1964. 
Maeda, H., Horizontal wind systems in the ionospheric E region deduced from the dynamo theory of the geomagnetic $\mathrm{Sq}$ variation Part I Nonrotating earth, J. Geomagnet. Geoelec., I, 121-132, 1955.

Malville, J. M., The effect of the initial phase of a magnetic storm upon the outen Van Allen belt, J. Geophys. Res., 65, 3008-3010, 1960.

Martyn, D. F., Atmospheric tides in the ionosphere I. Solar tides in the F2 region, Proc. Roy. Soc. A, 189, 241-260, 1947.

Martyn, D. F., Electric currents in the ionosphere III Ionization drift due to winds and electric fields, Phil. Trans. Roy. Soc., 246, $306-320,1954$.

Martyn, D. F., Theory of height and ionization density changes at the maximum of a Chapman-like region, taking into account of ion production, decay, diffusion, and tidal drift, The physics of the Ionosphere, Rep. Phys. Soc, London, Cavendish Lab. Cambridge, $254-259,1955$.

Nagata, T. and S. Kokubun, A particular geomagnetic daily variation ( ${ }_{q}^{p}$ ) in polar region on geomagnotically quiet day, Nature, 195, $555-557,2962$.

Ness, N. F., The earth's magnetospheric tail, Goddard Space Flight Center, Pub. X-612-64-392, November 1964 .

O'Brien, B. J., Lifetimes of outer-zone electrons and their precipitation into the atmosphere. J. Geophys. Res., 67, 3687-3706, 1962.

Parker, E. N., The hydrodynamic treatment of the expanding solar corona, Astrophys. J., 132, 175-183, 1960,

Parker, E. N., Coronal expansion and solar corpuscular radiation, Research in Geophysics (ed. by H. Odishaw) Vol. I, 99-114, 1964.

Pederson, P. O., The propagation of radio waves along the surface of the earth and in the atmosphere, Danmarks Naturvidenskabelige Samfund. A. Nr. $15 \mathrm{a} / \mathrm{b} 244+19 \mathrm{pp}$. Copenhagen, 1927.

Pekeris, C. L., Atmospheric oscillations, Proc. Roy. Soc. A, 158, 650-671, 1937.

Piddington, J. H., Geomagnetic stom theory, J. Geophys. Res., 65 , 93-106, 1960 .

Piddington, J. H., A hydromagnetic theory of geomagnetic storms and auroras, Planet. Space Sci., 9, 947-957, 1962.

Piddington, J. H., Theories of the geomagnetic storm main phase, Planet. Space Sci., 10, 1277-1288, 1963.

Prince, C. E., Jn., F. X. Bostick, Jr. and H. W. Smith, A study of the transmission of plane hydromagnetic waves through the upper atmosphere, Electrical Engineering Research Lab., Univ, of Texas, Rep. No. $134,1964$. 
Schlüter, A., Dyanmik des Plasmas II, Z. Natforschg., 69, 73-78, 1951.

Schuster, A., The diunnal variation of terrestrial magnetism, Phil.

Trans. London (A), 208, 163-204, 1908.

Simpson, G. C., Quar. Jour. Roy. Met. Soc., 44

Spreiter, J. R. and B. R. Briggs, Theory of electrostatic fields in the ionosphere at polar and middle geomagnetic latitudes, J. Geophys. Res., 66, $1731-1744,1961$.

Stewart, B., Terrestrial Magnetism, Ency. Brit. Sth ed., Pp. 36, 1882.

Taylor, G. I., The oscillations of the atmosphere, Proc. Roy. Soc. A, 256, 318-326, 1936.

Watanabe, T., Electrodynamical behaviour and screening effect, Sci. Rep. Tohoku Univ. Ser. 5, 81-98, 1957.

Williams, D. J. and W. F. Palmer, Distortions in the radiation cavity as measured by an 1100 kilometer polar orbiting satellite, J. Geophys. Res., 70, 557-567, 1965. 